\title{
Real-time MRI and Model-based Reconstruction Techniques for Parameter Mapping of Spin-lattice Relaxation
}

\author{
Dissertation \\ zur Erlangung des mathematisch-naturwissenschaftlichen \\ Doktorgrades \\ "Doctor rerum naturalium" \\ der Georg-August-Universität Göttingen \\ im Promotionsprogramm ProPhys \\ der Georg-August University School of Science (GAUSS)
}

vorgelegt von

Xiaoqing Wang

aus Jiangsu, China

Göttingen 2016 


\section{Betreuungsausschuss}

Prof. Dr. Jens Frahm

Prof. Dr. Hans Hofsäss
Biomedizinische NMR Forschungs GmbH

Max-Planck-Institut für biophysikalische Chemie (MPIBPC)

II. Physikalisches Institut (Atom- und Kernphysik)

Georg-August-Universität Göttingen

\section{Mitglieder der Prüfungskommission}

Referent/in:

Prof. Dr. Jens Frahm

Biomedizinische NMR Forschungs $\mathrm{GmbH}$

Max-Planck-Institut für biophysikalische Chemie (MPIBPC)

Korreferent/in: $\quad$ Prof. Dr. Hans Hofsäss

II. Physikalisches Institut (Atom- und Kernphysik)

Georg-August-Universität Göttingen

\section{Weitere Mitglieder der Prüfungskommission}

Prof. Dr. Thorsten Hohage Institut für Numerische und Angewandte Mathematik Georg-August-Universität Göttingen

Prof. Dr. Stefan Luther Biomedizinische Physik Max-Planck-Institut für Dynamik und Selbstorganisation (MPIDS)

Prof. Dr. Annette Zippelius Institut für Theoretische Physik Georg-August-Universität Göttingen

apl. Prof. Dr. Ulrich Parlitz Biomedizinische Physik Max-Planck-Institut für Dynamik und Selbstorganisation (MPIDS)

Tag der mündlichen Prüfung: 
To my family, for their endless love and support. 



\section{Contents}

\begin{tabular}{lll}
\hline 1 & Introduction & 1
\end{tabular}

2 Magnetic Resonance Imaging 5

2.1 Nuclear Spins and Magnetization . . . . . . . . . . . 5

2.1 .1 Excitation . . . . . . . . . . . . . 6

2.1 .2 Relaxation . . . . . . . . . . . . . 7

2.2 Signal Localization $\ldots \ldots \ldots \ldots$. . . . . . . . . . . 8

$2.2 .1 \quad$ Slice Selection . . . . . . . . . . . . . 8

2.2 .2 Frequency Encoding . . . . . . . . . . . . . 9

2.3 Pulse Sequence . . . . . . . . . . . . . . . . . . . 10

$2.3 .1 \quad$ Free Induction Decay $\ldots \ldots \ldots 10$

2.3 .2 Gradient Echo. . . . . . . . . . . . . . . . . . . . 11

2.3 .3 Spin Echo . . . . . . . . . . . . . . . . . 11

2.3 .4 Inversion Recovery . . . . . . . . . . . . . . . . . 12

$2.4 k$-space Trajectories . . . . . . . . . . . . . . . . . 13

2.5 Sampling Requirements . . . . . . . . . . . . . . 15

2.6 Image Reconstruction $\ldots \ldots \ldots$. . . . . . . . . . 17

2.6 .1 Gridding and FFT . . . . . . . . . . . . . . . . . . . . . . . . . . . . . . 17

2.6 .2 Iterative Reconstruction . . . . . . . . . . . . . 18

2.7 Parallel Imaging . . . . . . . . . . . . . . . . . 18

2.8 MRI System . . . . . . . . . . . . . . . . . . . . . 21

3 Real-time MRI 23

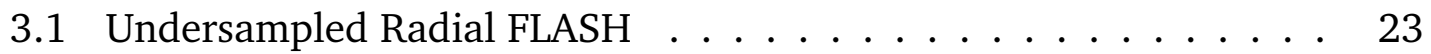

3.2 Parallel Imaging as Nonlinear Inverse Problem . . . . . . . . . . . 25

3.2 .1 Algorithms ....................... 25

3.2 .2 Preprocessing . . . . . . . . . . . . . . . 27

3.2 .3 Postprocessing $\ldots \ldots \ldots \ldots$. . . . . . . . . . . . . . 27

4 Introduction to Quantitative T1 Mapping Techniques 29

4.1 Introduction . . . . . . . . . . . . . . . . . . . . 29

4.2 Inversion Recovery T1 mapping . . . . . . . . . . . . . . . . 29

4.3 Inversion Recovery Look-Locker T1 mapping . . . . . . . . . . . . . 31 
4.4 Variable Flip Angle T1 mapping . . . . . . . . . . . . . . . . . . 32

5 Single-shot Inversion-recovery Look-Locker T1 Mapping by Real-time

MRI

5.1 Introduction . . . . . . . . . . . . . . . . 35

5.2 Materials and Methods . . . . . . . . . . . . . . . . . 36

$5.2 .1 \quad$ Single-shot IR LL Radial FLASH $\ldots$. . . . . . . . . . . . . 36

5.2 .2 Image Reconstruction . . . . . . . . . . 37

5.2 .3 T1 Quantitation . . . . . . . . . . . . . . 37

5.2 .4 Numerical Simulations . . . . . . . . . . . . . . . . 38

$5.2 .5 \mathrm{MRI} \ldots \ldots \ldots \ldots \ldots$

5.3 Results . . . . . . . . . . . . . . . . . . . . 39

$5.3 .1 \quad$ Numerical Simulations . . . . . . . . . . . . . . 39

5.3 .2 in vitro Studies . . . . . . . . . . . . . . . . . . . 43

5.3 .3 in vivo Studies .................. . . 43

5.4 Extensions . . . . . . . . . . . . . . . . . . . 48

$5.4 .1 \quad$ Modified Non-local Means (NLM) Filter . . . . . . . . . . . 48

5.4 .2 Single-shot Multi-slice T1 mapping . . . . . . . . . . . 50

5.5 Discussion . . . . . . . . . . . . . . . . . 54

5.6 Summary . . . . . . . . . . . . . . . 57

6 Single-shot Myocardial T1 Mapping by Real-time MRI 59

6.1 Introduction . . . . . . . . . . . . . . . . . 59

6.2 Methods . . . . . . . . . . . . . . . . . 60

6.2 .1 MRI Acquisition and Reconstruction . . . . . . . . . . . . 60

6.2 .2 T1 Quantitation . . . . . . . . . . . . . . . 62

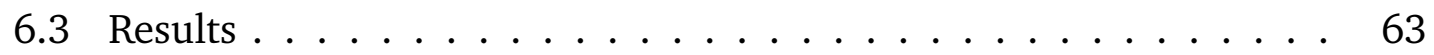

6.4 Discussion . . . . . . . . . . . . . . . . . . . 69

6.5 Summary . . . . . . . . . . . . . . . . 71

7 Model-based Reconstruction for Single-shot Inversion-recovery Look$\begin{array}{ll}\text { Locker T1 Mapping with Sparsity Constraints } & 73\end{array}$

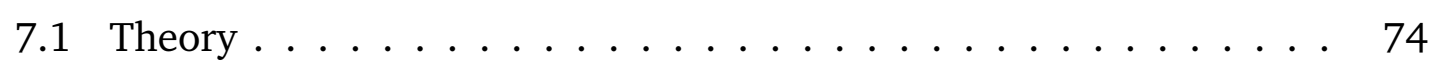

7.1 .1 Signal Equation . . . . . . . . . . . . . . 74

7.1 .2 Model-based Reconstruction . . . . . . . . . . . . . 74

7.1 .3 Regularization ................... 75

7.1 .4 Optimization .................... 76

7.2 Materials and Methods . . . . . . . . . . . . . . . . . 78

$7.2 .1 \quad$ Data Acquisition $\ldots \ldots \ldots \ldots 78$

7.2 .2 Numerical Simulations . . . . . . . . . . . 78 
$7.2 .3 \quad$ MRI $\ldots \ldots \ldots \ldots \ldots$

7.2 .4 Parameter Choice for Reconstruction . . . . . . . . . . . 79

7.2 .5 Implementation. . . . . . . . . . . . . . . . 81

7.3 Results . . . . . . . . . . . . . . . . . . . . 81

7.3 .1 Validation Studies $\ldots \ldots \ldots \ldots$. . . . . . . . . . 81

7.3 .2 Human Studies . . . . . . . . . . . . . . . . . . . . 86

7.4 Discussion . . . . . . . . . . . . . . . . . . . . . . . . 92

7.5 Summary $\ldots \ldots \ldots \ldots \ldots \ldots \ldots \ldots$

7.6 Appendix $\ldots \ldots \ldots \ldots \ldots \ldots$

7.6.1 Derivative and adjoint derivatives of the operators . . . . . 94

\begin{tabular}{lll}
\hline & Summary and Outlook & 97
\end{tabular}

8.1 Summary . . . . . . . . . . . . . . . . . . 97

8.2 Future Work . . . . . . . . . . . . . . . . . . . . . 98

\begin{tabular}{ll}
\hline Appendix & 101
\end{tabular}

\begin{tabular}{ll}
\hline Bibliography & 103
\end{tabular}

\begin{tabular}{ll}
\hline Acronyms & 119
\end{tabular}

\begin{tabular}{ll}
\hline List of Publications & 121
\end{tabular}

\begin{tabular}{ll}
\hline Acknowledgements & 123
\end{tabular} 



\section{Introduction}

Magnetic resonance imaging (MRI) is a non-invasive technique widely used for imaging of humans and animals. It came into existence based on several fundamental inventions made in the 1970s. Since then, MRI has evolved into one of the leading tools in biomedical research and clinical diagnostics. Compared to other medical imaging modalities such as X-ray computed tomography (CT) or positron emission tomography ( $\overline{\text { PET) }}$, MRI uses neither ionizing radiation nor radioactive materials and offers a rich set of image contrasts based on signal relaxation times $T_{1}$ and $T_{2}$, spin diffusion or more complex quantities such as tissue perfusion. Moreover, the acquisition of images with multiple contrasts in the same anatomical region enables the capability to obtain quantitative maps of the underlying relaxation parameters, which renders the MRI scanner not only a camera, but also a scientific measuring instrument. This thesis specifically addresses the quantitative mapping of $T_{1}$ relaxation times.

Quantitative $T_{1}$ mapping normally consists of a suitable magnetization preparation (e.g., inversion), followed by the acquisition of a predefined number of images of the relaxation process. After data acquisition and image reconstruction, the relaxation model is then fit to the images to obtain the parameter maps. However, the acquisition of multiple images with a spatial and temporal resolution that is sufficient for clinical use with only one preparation may be difficult or even impossible in presence of short relaxation times. One way to overcome this problem is a segmentation of the data acquisition process. By acquiring complementary data subsets at different segmentations, the temporal resolution can be highly improved. For segmented acquisitions with preparation by inversion, a sufficient delay time is required between the end of one segment and a following inversion for the next acquisition. This delay period largely prolongs the total data acquisition time and restricts the in vivo applications of $T_{1}$ mapping techniques. Therefore, novel ways that allow for the reconstruction of accurate high-resolution $T_{1}$ maps with less data sampled than required by the Nyquist criterion, known as undersampling, would be highly desirable.

In the last two decades, several techniques have been proposed to address the general problem of the long MRI acquisition times which are caused by the pointby-point data acquisition scheme in Fourier space. One approach is parallel imag- 
ing which uses multiple receive coils to acquire data in parallel. By exploiting complementary spatial information from these spatially distinct receiver coils, parallel imaging typically allows for a moderate reduction of spatial encoding steps (i.e., acceleration) by a factor of 2-4. Another effort to improve imaging speed is the development of non-Cartesian MRI. Especially the adoption of radial sampling has gained a lot of interest as it is inherently robust to motion and at least partly tolerant to undersampling. More specifically, the combination of highly undersampled radial fast low angle shot (FLASH) acquisitions, parallel imaging and image reconstruction by nonlinear inversion (NLINV) which jointly estimates the image and all coil sensitivity maps has achieved real-time MRI at millisecond resolution. Moreover, when calculating quantitative maps of relaxation parameters, a lot of redundancy may be found in the data of image series with different contrasts. Model-based reconstruction techniques promise to exploit such redundancies by directly reconstructing parameter maps from raw data. Although this approach comes at a cost of increased complexity and computational demand, it has been successfully demonstrated in preliminary applications to $T_{2}$ mapping.

The main focus of this thesis is to develop new methods for fast and accurate $T_{1}$ mapping at high spatial resolution by taking advantage of both the above mentioned real-time MRI developments and the concept of a model-based reconstruction. The former method reconstructs images from highly undersampled radially encoded datasets, which then may be followed by a pixel-wise fitting to obtain parameter maps. The latter, on the other hand, bypasses the intermediate steps of image reconstruction and pixel-wise fitting by estimating parameter maps directly from the undersampled raw data with use of a known signal model.

As far as organization of this thesis is concerned, Chapter 2 discusses the basic principles of MRI, while Chapter 3 briefly explains undersampled radial FLASH acquisitions and NLINV reconstruction algorithms as the main components of realtime MRI. Chapter 4 introduces the three most commonly used experimental approaches to quantitatively measure the $T_{1}$ relaxation process. The main achievements of this thesis are presented in Chapters 5 to 7 .

Chapter 5 deals with the development of a single-shot high-resolution $T_{1}$ mapping technique based on real-time MRI. Apart from the adaptation of the highly undersampled radial FLASH data acquisition technique, the image reconstruction involved a modified NLINV-based algorithm. After optimization of acquisition and reconstruction parameters, the single-shot $T_{1}$ mapping method could be further developed to a simultaneous multi-slice technique which yields $T_{1}$ maps of multiple slices within a single experiment, i.e., within a few seconds as needed for a single inversion-recovery experiment.

Chapter 6 presents a clinically relevant extension of the above method to single- 
shot diastolic myocardial $T_{1}$ mapping where systolic images are automatically masked out prior to pixel-wise fitting. Robustness and reproducibility of this and the previous method have been validated with use of a numerical phantom, and experimental phantom and human subjects in vivo.

Chapter 7 introduces and evaluates the proposed model-based reconstruction technique which estimates the parametric maps of a suitable signal model and all coil sensitivities directly from the undersampled raw data. A golden-angle data acquisition scheme together with random $\mathrm{RF}$ spoiling was applied to efficiently sample the data. The joint estimation of unknowns is formulated as a nonlinear inverse problem where a priori information (i.e., sparsity constraints) of the parameter maps is incorporated into the reconstruction process to improve $T_{1}$ precision. Validations again included numerical simulations, an experimental phantom and in vivo studies of healthy human subjects. A comparison of the model-based reconstruction to the method introduced in Chapter 5 is also presented. 



\section{Magnetic Resonance Imaging}

This chapter introduces the basic principles of nuclear magnetic resonance (NMR) and magnetic resonance imaging (MRI). It briefly covers the generation of MR signals, signal types, spatial encoding, image reconstruction and parallel imaging. For comprehensive information about MRI, it is recommended to refer to the textbooks by Haacke et al. [1], Liang et al. [2] and Bernstein et al. [3].

\subsection{Nuclear Spins and Magnetization}

The NMR phenomenon was first described and measured by Isidor Rabi in 1938 [4] and then expanded by Felix Bloch and Edward Mills Purcell in 1946 [5, 6]. A fundamental property of nuclei is that those with odd atomic weights and/or odd atomic numbers, such as ${ }^{1} \mathrm{H},{ }^{13} \mathrm{C}$ and ${ }^{23} \mathrm{Na}$, poss an angular momentum $\boldsymbol{J}$, which is often called spin. Like any spinning charged object, a nucleus with a nonzero spin creates a magnetic field around it. It is represented by a vector quantity $\boldsymbol{\mu}$ and is called the nuclear magnetic dipole moment or magnetic moment. The spin angular momentum and the magnetic moment are related by:

$$
\boldsymbol{\mu}=\gamma \boldsymbol{J}
$$

where $\gamma$ is the gyromagnetic ratio. Although the magnitude of $\boldsymbol{\mu}$ is certain, its direction is completely random due to thermal random motion. This will result in a zero net magnetization around a macroscopic object at thermal equilibrium. In the presence of an external magnetic field $B_{0}$, the nuclear spins experience the Zeeman splitting phenomenon. For hydrogen protons, they are aligned in two energy states with energy values proportional to the external magnetic field:

$$
E_{\uparrow}=-\gamma \frac{\hbar}{2} B_{0} \quad E_{\downarrow}=\gamma \frac{\hbar}{2} B_{0}
$$

where $\hbar$ is the Planck's constant divided by $2 \pi$. The states correspond to a parallel $(\uparrow)$ or anti-parallel $(\downarrow)$ alignment of the magnetic moments $\boldsymbol{\mu}$ with the external 
magnetic field $B_{0}$. The energy difference between the two spin states is given by

$$
\Delta E=E_{\downarrow}-E_{\uparrow}=\gamma \hbar B_{0} .
$$

According to the Boltzmann relationship, the spin population difference between these two states yields:

$$
\frac{N_{\uparrow}}{N_{\downarrow}}=\exp \left(\frac{\Delta E}{K_{b} T}\right)
$$

where $K_{b}$ and $T$ are the Boltzmann constant and temperature respectively. Although it is small, the population difference generates an observable macroscopic magnetization $\vec{M}$ from the spin system. The resulting bulk magnetization vector $\vec{M}$ points exactly along the positive direction of the external field. Its magnitude is given by:

$$
M_{0}=|\vec{M}|=\frac{\gamma^{2} \hbar^{2} N_{s}}{4 K_{b} T} B_{0} .
$$

The above equation 2.5 indicates that the magnitude of $\vec{M}$ is proportional to the external magnetic field $B_{0}$ as well as to the number of spins $N_{s}$.

In addition, there are two important properties about nuclear precession, one is the angular frequency of nuclear precession:

$$
\omega_{0}=\gamma B_{0}
$$

which is known as the Larmor frequency. Second, precession of $\boldsymbol{\mu}$ around $B_{0}$ is clockwise if observed against the direction of the magnetic field.

\subsubsection{Excitation}

The bulk magnetization vector $\vec{M}$ experiences a torque with the application of a magnetic field $\vec{B}_{1}$ according to the classical equation of motion:

$$
\frac{d \vec{M}}{d t}=\gamma \vec{M} \times \overrightarrow{B_{1}}
$$

In pulsed NMR experiments (Figure 2.1), a radio frequency (RF) field $\overrightarrow{B_{1}}$ with frequency $\omega_{0}$ is used to excite the spins. $\vec{B}_{1}$ is oriented perpendicular to $z$, the resulting torque is perpendicular to $\vec{M}$. During the excitation, the magnetization vector $\vec{M}$ is tilted towards the xy-plane where it precesses with $\omega_{0}$. The flip angle $\alpha$ is proportional to integral over the envelope $B_{1}(t)$ of the pulse:

$$
\alpha=\gamma \int_{0}^{T_{\mathrm{rf}}} B_{1}(t) d t
$$


with $T_{\mathrm{rf}}$ the rf-pulse duration. A pulse which rotates the magnetization vector by a certain flip angle $\alpha$ is often referred to as an $\alpha$-pulse.

After the application of the $\alpha$-pulse, a $M_{x y}$ component of the magnetization precesses around the $z$-axis. The time varying magnetic field induces an oscillating signal in a suitable positioned nearby receiver coil. This is the NMR signal used for detection and quantification of the proton spin-density in MRI.

Equilibrium

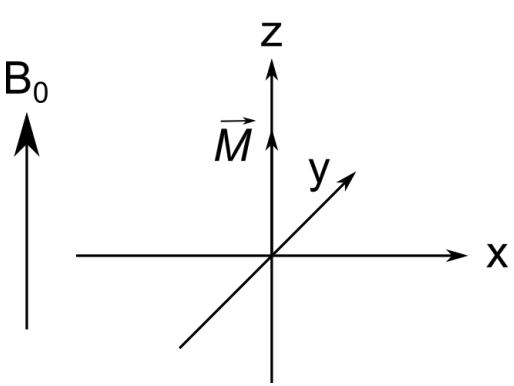

Excitation

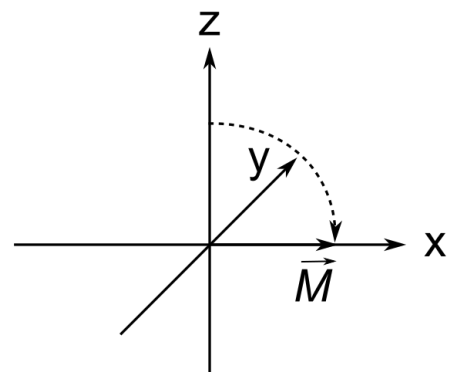

Precession

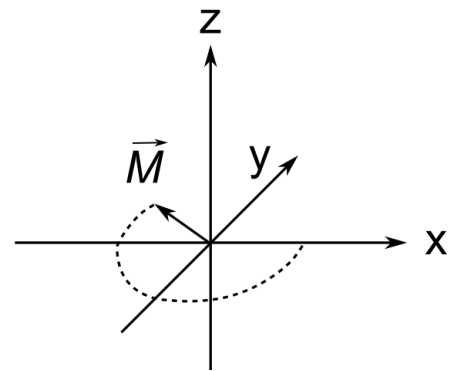

Figure 2.1: Representation of the pulsed NMR experiment: In equilibrium, the magnetic moments align themselves along the static magnetic field $B_{0}$. After excitation with a RF pulse the spins are tilted into the xy-plane and precess with the Larmor frequency.

\subsubsection{Relaxation}

After RF excitation, the signal detected declines rapidly due to spins proceeding towards the thermal equilibrium state. This process can be expressed by the equation expanded from (2.7) by Bloch in 1946 [5]:

$$
\frac{d}{d t} \vec{M}=\gamma \vec{M} \times \vec{B}+\left(\begin{array}{c}
-\frac{1}{T_{2}} M_{x} \\
-\frac{1}{T_{2}} M_{y} \\
\frac{M_{\mathrm{eq}}-M_{z}}{T_{1}}
\end{array}\right) .
$$

The constant $T_{1}$ is the spin-lattice relaxation time which describes the relaxation of the longitudinal magnetization caused by energy exchange between the protons and the surrounding environment. The constant $T_{2}$ is called spin-spin relaxation time and describes the loss of transversal magnetization. Since the decrease in transversal magnetization is not only caused by the exchange of energy with the environment but also by energy changes between spins, $T_{2}$ is smaller than $T_{1}$.

To simplify the analysis, the two components of the transversal magnetization $M_{x}$ and $M_{y}$ are usually combined into one single complex vector

$$
M_{\perp}(t)=M_{x}(t)+i M_{y}(t)
$$


In the absence of $B_{1}$ field, equation (2.9) with regard to $M_{\perp}$ and $M_{z}$ is given by:

$$
\begin{aligned}
M_{\perp}(t) & =M_{\perp}(0) e^{-t / T_{2}} \\
M_{z}(t) & =M_{\text {eq }}+\left(M_{z}(0)-M_{\text {eq }}\right) e^{-t / T_{1}}
\end{aligned}
$$

where $M_{\perp}(0)$ and $M_{z}(0)$ are the transversal and longitudinal magnetization directly after $\mathrm{RF}$ excitation respectively. The influence of the relaxation constants can be controlled by the parameters of the NMR or MRI experiment. Therefore signals with different contrast can be generated. Because of their dependence on the individual sample material, $T_{1}$ and $T_{2}$ offer a powerful tool to distinguish human tissues in clinical MRI.

\subsection{Signal Localization}

So far the detected MRI signal is proportional to the density of all excited proton spins within the reception range of the $\mathrm{RF}$ coil. To allow for two- or threedimensional imaging, it is necessary to distinguish them from different spatial locations. Spatial encoding is therefore introduced. There are two basic methods/principles for spatial encoding in MRI. One is slice selection, where only one slice is excited. The other one is Fourier encoding, which can be used to encode the signal of the excited spins. In 2D imaging, a slice is selected and the remaining 2D plane is Fourier encoded. In 3D imaging, Fourier encoding is employed for all three dimensions.

The spatial encoding is achieved with the use of additional gradient coils along with main magnetic field $B_{0}$ as introduced by Lauterbur in 1973 [7]. These gradient coils create gradient fields in $x, y$ and $z$ directions. By switching them either separately or in combination with each other, a spatial dependent magnetic field can be created:

$$
\vec{B}(\vec{r}, t)=\overrightarrow{B_{0}}+\overrightarrow{G(t)} \cdot \vec{r}=\left(\begin{array}{c}
0 \\
0 \\
B_{0}
\end{array}\right)+\left(\begin{array}{c}
G_{x} \\
G_{y} \\
G_{z}
\end{array}\right) \cdot\left(\begin{array}{c}
x \\
y \\
z
\end{array}\right)
$$

with the gradient fields $G_{x}, G_{y}, G_{z}$ in three spatial directions respectively.

\subsubsection{Slice Selection}

To excite only a slice of the sample, an external field gradient is switched on during the excitation pulse. According to the Larmor equation (2.6), the resonance 
frequency then varies linearly along the gradient direction, e.g., $z$ direction,

$$
\omega(z)=\gamma B(z)=\gamma\left(B_{0}+G_{z} \cdot z\right)
$$

With the application of the excitation pulse (Figure 2.2) which carries a bandwidth of frequencies $\Delta \omega$, only spins with the corresponding resonance frequencies will be excited. This reduces the image plane from $3 \mathrm{D}$ to $2 \mathrm{D}$. In addition, to excite a rectangular-shaped slice in the spatial frequency domain, a sinc shape pulse needs to be used in the time domain, In practice, because the sinc function has an unlimited support, a truncated sinc function is used instead with additional filtering to achieve finite length.
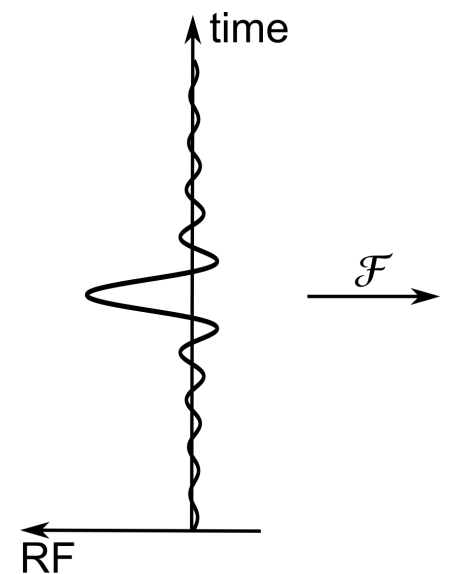

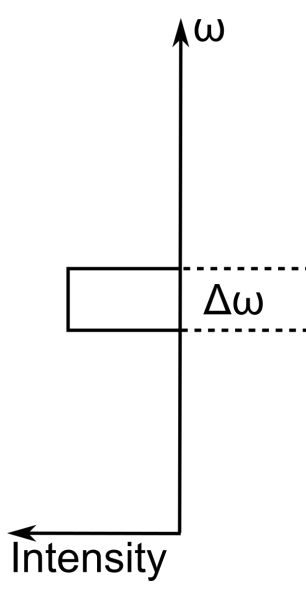

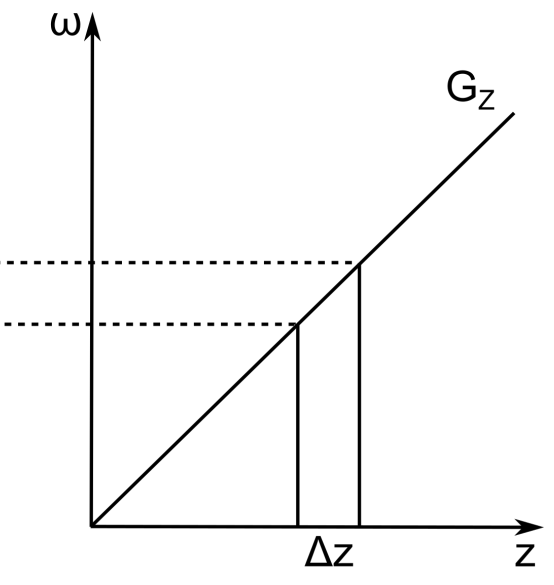

Figure 2.2: Slice selection: A slice selection gradient $G_{z}$ leads to a linearly varying resonance frequency of the spins along $z$ direction. A sinc RF pulse then ideally excites a slice with rectangular profile. $\mathcal{F}$ represents Fourier transform.

\subsubsection{Frequency Encoding}

Two field gradients (e.g., $G_{x}$ and $G_{y}$ ) are used to encode signals from the same slice. Similar to equation (2.14), the resonance frequency depends on the position of the spin:

$$
\omega_{\perp}=\gamma \vec{G} \cdot \vec{r}=\gamma\left(G_{x} x+G_{y} y\right)
$$

An additional phase term is generated on the transverse magnetization with the application of above gradient fields:

$$
M_{\perp}(\vec{r}, t)=M_{0}(\vec{r}) \cdot e^{-i\left(\omega_{0} t+\varphi(\vec{r}, t)\right)}
$$


where the phase evolution $\varphi$ depends on the time integral over the gradients:

$$
\varphi(\vec{r}, t)=\gamma \int_{0}^{t} \vec{G}(\tau) d \tau \cdot \vec{r}=2 \pi \vec{k} \cdot \vec{r}
$$

and $\vec{k}(t)$ is the $k$-space trajectory:

$$
\vec{k}(t):=\frac{\gamma}{2 \pi} \int_{0}^{t} \vec{G}(\tau) d \tau
$$

The real-valued signal detected by a large surrounding coil can be calculated by integrating the transverse magnetization over the complete volume:

$$
\mathbb{R} \int M_{\perp}(\vec{r}, t) d \vec{r}=\mathbb{R} \int M_{\perp}(\vec{r}, 0) e^{-2 \pi i \vec{k} \cdot \vec{r}} e^{-i \omega_{0} t} d \vec{r} .
$$

The high frequency phase $e^{-i \omega_{0} t}$ is then removed by quadrature demodulation. Assuming the initial magnetization $M_{\perp}(\vec{r}, 0)$ is proportional to the spin density $\rho(\vec{r})$, the signal received from the MRI scanner reads:

$$
s(t)=a \cdot \int \rho(\vec{r}) e^{-2 \pi i \vec{k}(t) \cdot \vec{r}} d \vec{r} .
$$

Here $a$ is a spatially invariant constant. The above equation reveals that the MRI signal corresponds to the Fourier transform of the proton density $\rho(\vec{r})$.

\subsection{Pulse Sequence}

A pulse sequence is used for generation of a useful MR signal for detection. The most important sequences are described in the following sections.

\subsubsection{Free Induction Decay}

After RF excitation, the excited spins send out a signal with Larmor frequency. Because local field inhomogeneities also contribute to the dephasing of spins, the signal decays exponentially with an effective spin-spin relaxation time $T_{2}^{*}$ (Figure 2.3), which is smaller than $T_{2}$ :

$$
\frac{1}{T_{2}^{*}}=\frac{1}{T_{2}}+\frac{\gamma \Delta H}{2}
$$

with $\Delta H$ describing both the static field inhomogeneity and the effect of the magnetic field gradients [8]. 

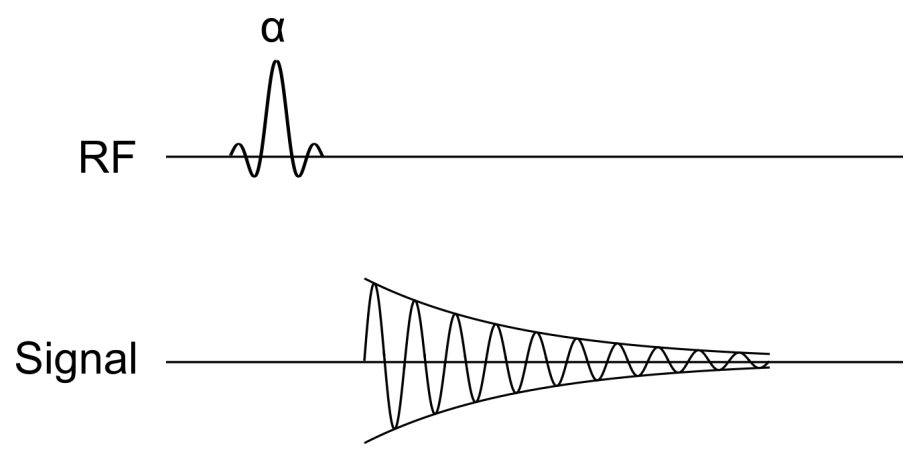

Figure 2.3: Free induction decay (FID): The signal send out from the excited spins decays according to $T_{2}^{*}$ relaxation.

\subsubsection{Gradient Echo}

The gradient echo sequence consists of two gradients with opposite polarity namely prephasing and readout gradient. The spins are initially dephased by the prephasing gradient and then are rephased with a readout gradient to generate an echo (Figure 2.4). Because spins are partially rephased due to a combined effect of field inhomogeneity and $T_{2}$ relaxation, the signal strength depends on the echo time TE according to $\exp \left(-\mathrm{TE} / T_{2}^{*}\right)$. The gradient echo is the base of the FLASH imaging sequence [9].

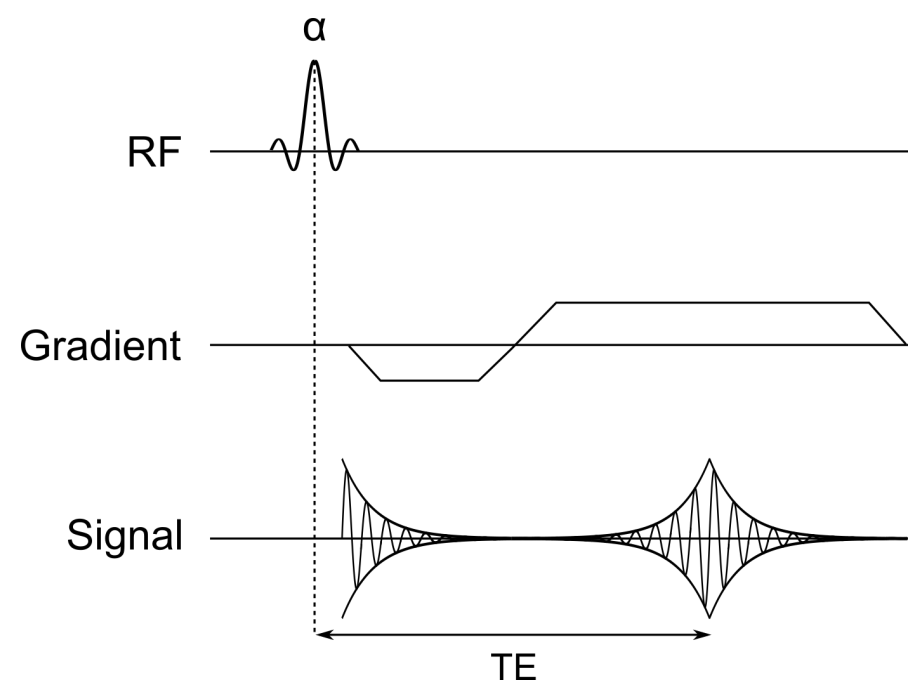

Figure 2.4: Gradient echo (GRE): After dephasing of the spins with a gradient, an echo can be created by rephasing with a gradient of opposite polarity.

\subsubsection{Spin Echo}

The spin echo sequence was introduced by Hahn in 1950 [10]. The basic pulse sequence consists of the application of two RF pulses with different flip angles $90^{\circ}$ and $180^{\circ}$ (Figure 2.5). Similar to gradient echo, the magnetization is flipped by the 


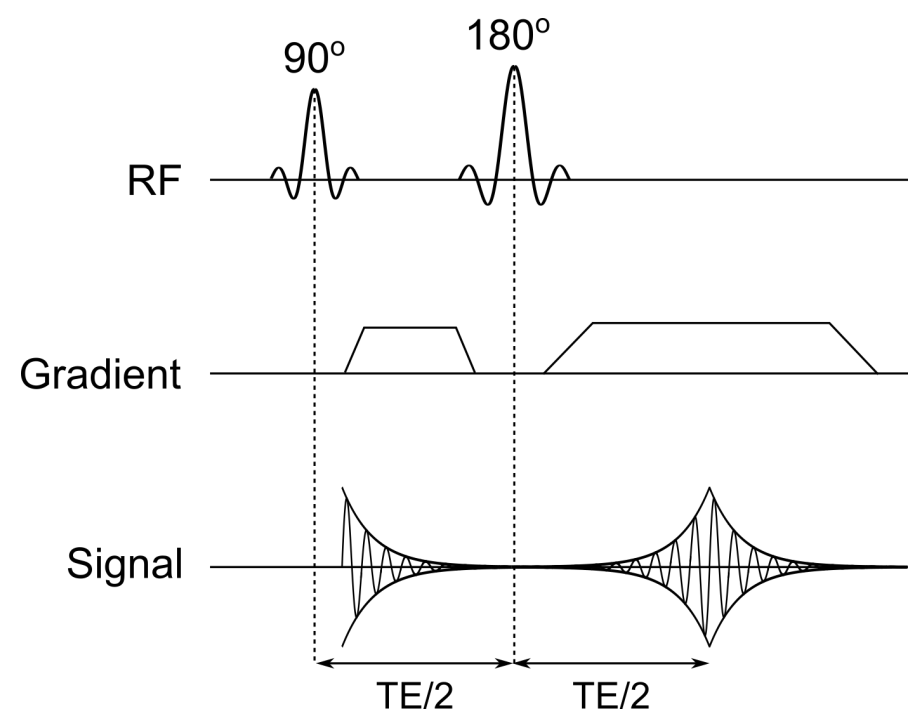

Figure 2.5: Spin echo (SE): Dephased spins are rephased with a $180^{\circ}$ pulse which rotates all spins by $180^{\circ}$ around the $\mathrm{x}$-axis. The phase of the transversal magnetization is then exactly inverted.

$90^{\circ}$ pulse to the transverse plane and dephases with time. With the application of the $180^{\circ}$ pulse, the accumulated phase differences between different spins subject to different magnetic fields are inverted. After the same time the spins are again exactly in phase and a spin echo is generated. The spin echo phase differences created by the gradient as well as by local field distortions are compensated by this process. Therefore, the signal amplitude depends on the $T_{2}$ relaxation time instead of $T_{2}^{*}$.

\subsubsection{Inversion Recovery}

Inversion recovery pulse sequences are used to manipulate signal contrast due to variations in $T_{1}$ relaxation time. An inversion recovery pulse sequence consists of two parts (Figure 2.6). The inversion pulse first nutates the equilibrium magnetization vector from the $+\mathrm{z}$ axis to the $-\mathrm{z}$ axis. After a waiting time (known as inversion time, denoted by TI) of $T_{1}$ relaxation, the second part is played out, which is typically self-contained pulse sequence such as RF spin-echo or gradient echo introduced above.

The inversion of magnetization is normally accomplished by employing an adiabatic inversion pulse [11--14]. Adiabatic inversion pulses operate under the adiabatic passage principle, which states that a magnetization vector initially parallel to the effective magnetic field follows the direction of the effective magnetic field, provided the effective field does not change its direction much during one rotational period of the magnetization about the effective field. To satisfy the above condition, the amplitude and frequency of the RF pulse are modulated to ensure 
Inversion

pulse

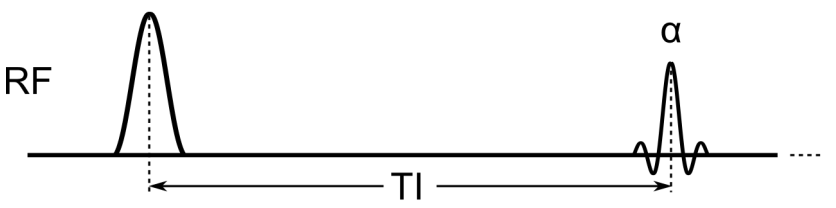

Remainder of

the pulse

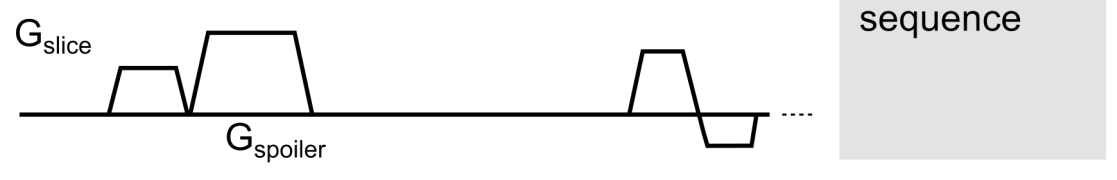

- IR module -

Host sequence

Figure 2.6: A generic inversion recovery (IR) pulse sequence consisting of an IR module and a host sequence, separated by inversion time TI. A selective inversion pulse is shown, but a nonselective pulse can also be used. The host sequence can be RF spin-echo, gradient echo, or others.

a relatively long pulse width and a rather high $B_{1}$ amplitude. The resulting magnetization vector becomes immune to the $B_{1}$ field variations, leading to a spatially uniform inversion of the magnetization. For more details of adiabatic pulses, see chapter 6 in [3]. The generic adiabatic inversion process is demonstrated in Figure 2.7
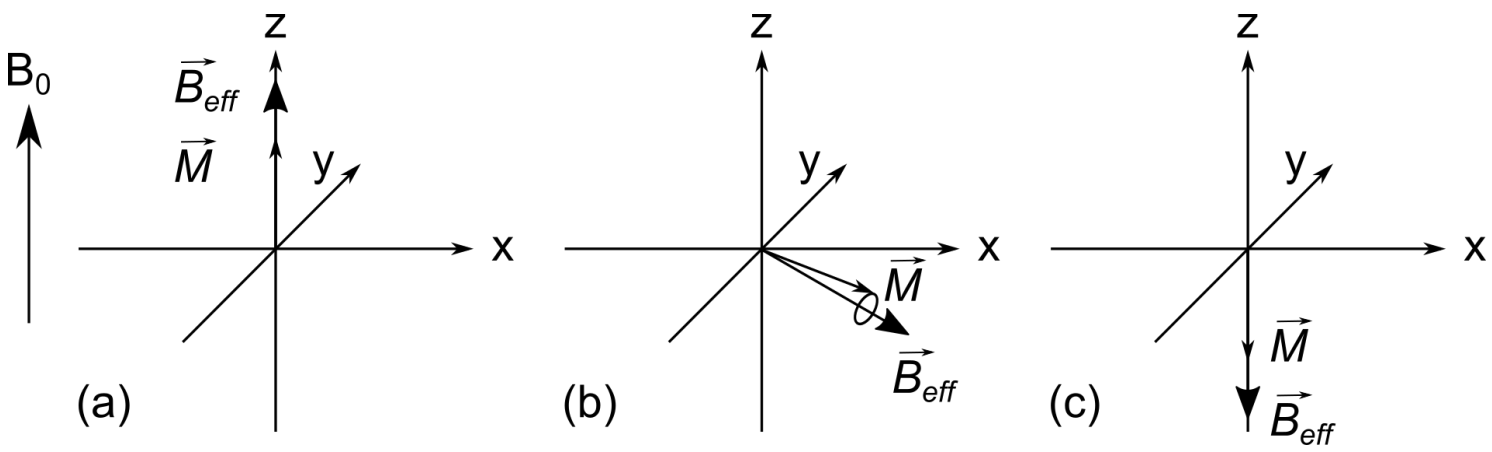

Figure 2.7: The inversion process in an adiabatic pulse. (a) Beginning of the adiabatic inversion pulse; (b) during the application of the adiabatic pulse. The magnetization $\overrightarrow{\boldsymbol{M}}$ precesses in a tight cone about the effect field $\vec{B}_{e f f} ;$ (c) at the end of the adiabatic pulse.

\section{$2.4 k$-space Trajectories}

In principle, any $k$-space trajectory can be generated by switching gradients along time according to equation (2.18). Figure 2.8 depicts the most common used ones. Figure 2.8(a) is the conventional Cartesian sampling scheme, in which $k$ space is sampled line-by-line. Since every sample is located on the Cartesian grid, 


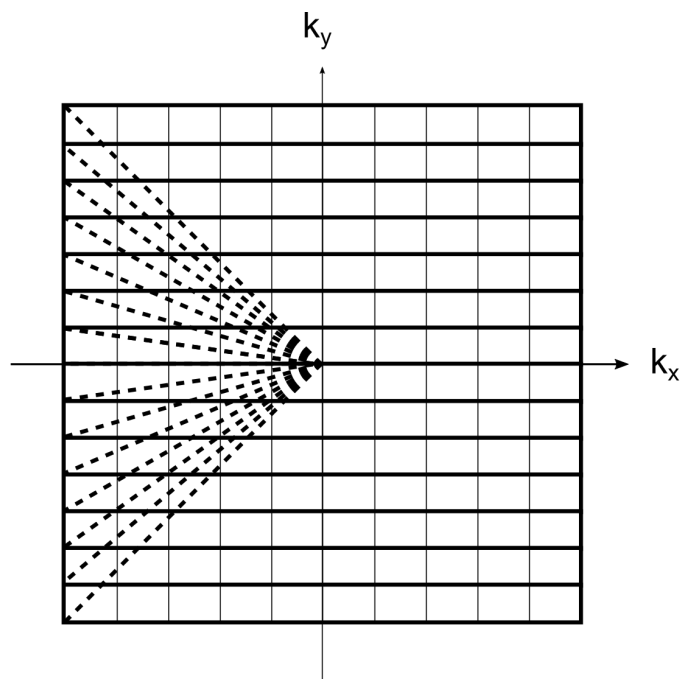

(a)

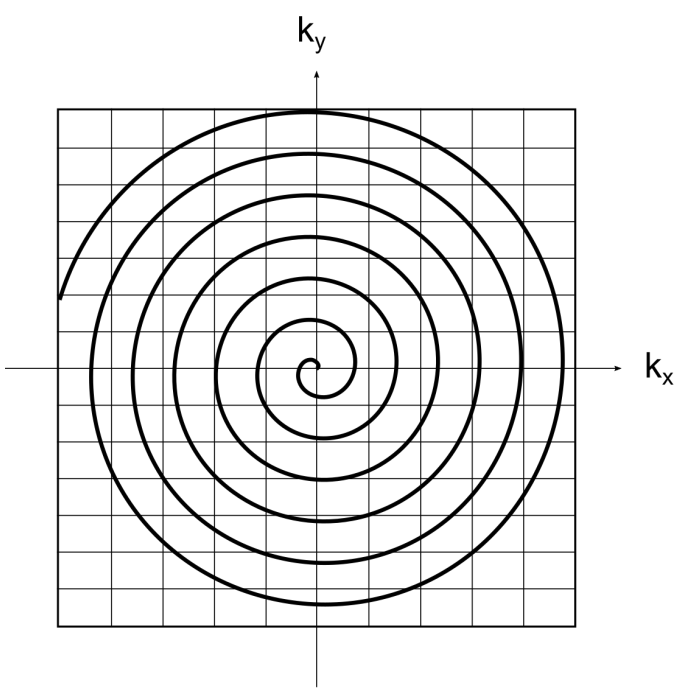

(c)

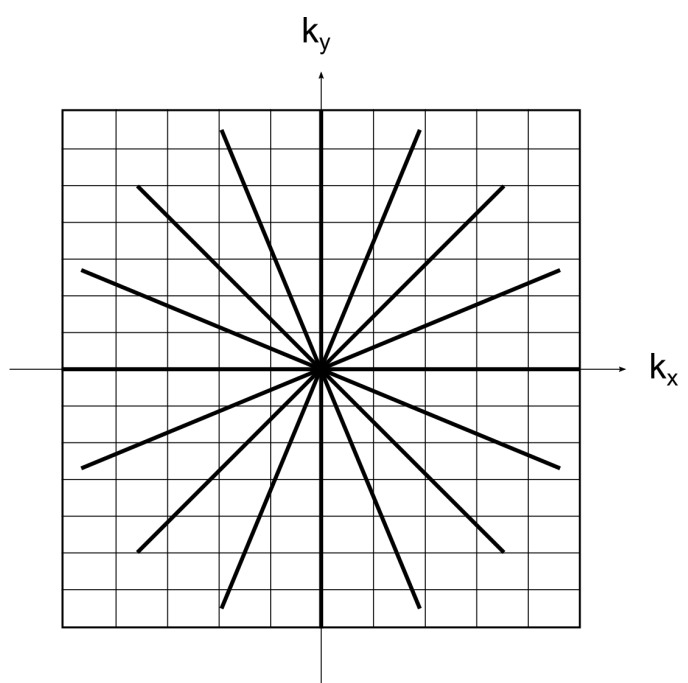

(b)

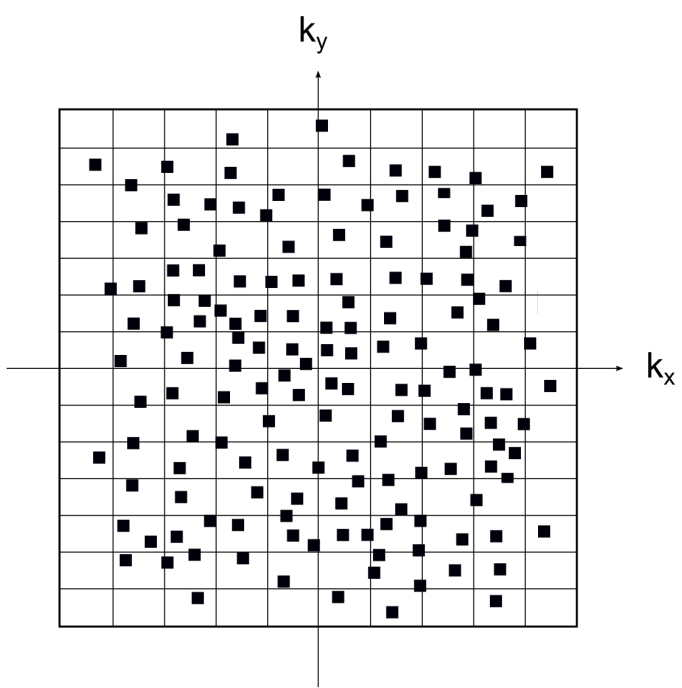

(d)

Figure 2.8: Schematic representation of four typical sampling patterns used in MRI studies: (a) Cartesian (b) Radial (c) Spiral (d) Random. 
this allows for simple reconstruction with a Fast Fourier Transformation (FFT). Moreover, this sampling scheme is insensitive to gradient imperfections. Due to these advantages, the Cartesian sampling scheme is still the most widely used in todays' clinical routine. A Cartesian FLASH sequence is demonstrated in Figure 2.9 .

The radial trajectory is demonstrated in Figure 2.8(b), this sampling scheme is originally proposed by Lauterbur in 1973, it is accomplished by setting the $x$ - and $y$-gradient according to:

$$
G_{x}=G_{\max } \cdot \cos (\theta) \quad G_{y}=G_{\max } \cdot \sin (\theta)
$$

where $G_{\max }$ denotes the amplitude required for sampling the central $k$-space row, $\theta$ is the desired angle of the $k$-space line (spoke). The radial sampling scheme requires a more precise gradient switching scheme which was a major limitation due to hardware imperfections in the early days of MRI. Nowadays it is becoming more and more popular because it combines the advantages of line-by-line scanning with better undersampling behavior and is more robust to motion. More discussions see [15].

Spiral sampling trajectory is illustrated in Figure 2.8(c), this method is also attractive because of its imaging speed. However, the use of long trajectory is problematic because the $T_{2}$ relaxation and off-resonance effects caused by field inhomogenities will lead to blurring and phase variations in the reconstructed images. For more details see [16].

Random sampling in Figure 2.8(d) is another sampling trajectory which becomes more popular in the MRI society due to the arise of the compressed sensing (CS) [17] theory. It is employed in order to meet the incoherence criterion which is one of the prerequisites to successfully apply the CS theory for fast MRI [18]. Since the random sampling in 2D is inefficient in MRI, 3D sampling is more commonly used. In the later case, one direction of gradient (e.g., $G_{z}$ ) is used as a readout gradient, the other two gradients $\left(G_{x}\right.$ and $\left.G_{y}\right)$ are switched randomly to meet the criterion of incoherent sampling.

\subsection{Sampling Requirements}

In practice, the continuous $k$-space signal needs to be cut off and sampled discretely at a certain sampling rate. The sampling process can be seen as a multiplication of the continuous $k$-space signal of the object with a Dirac comb function. According to the convolution multiplication property [19], multiplication with a Dirac comb function in image space corresponds to a convolution with the Fourier 

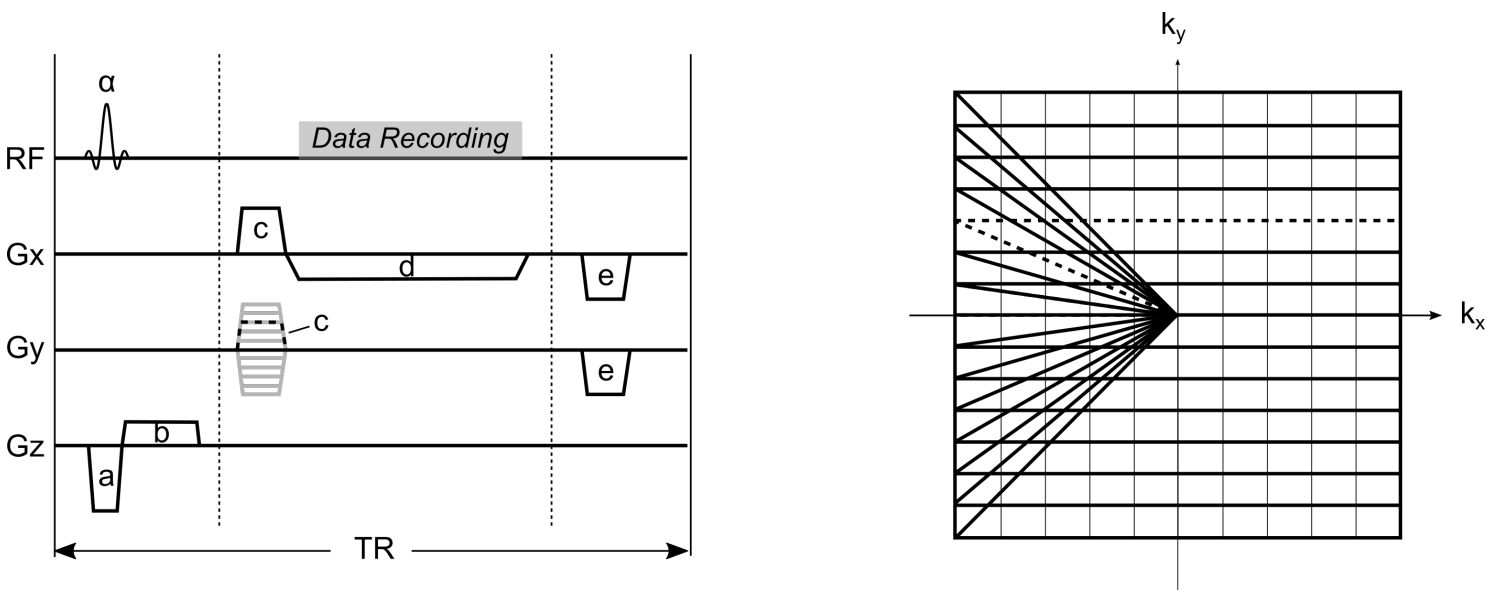

Figure 2.9: (Left) Timing diagram of a generic FLASH MRI sequence and (right) the respective sampling trajectory (dashed line corresponds to one cycle of the sequence). (a) Slice-selection, (b) rewinder gradient, (c) prephasing, (d) readout, (e) spoiler.

transformation of the Dirac comb-function which is another comb-function with reciprocal interval width in Fourier space. Hence, the discrete sampling leads to occurrence of periodic object copies, where the distance between the copies is reciprocal to the sample distance in $k$-space. In order to avoid image overlap, or so-called aliasing artifact, the maximum distance of samples in $k$-space $\Delta k$ and the desired Field of View (FOV) of the object should satisfy:

$$
\Delta k \leq \frac{1}{\mathrm{FOV}}
$$

The theory of the above sampling process follows the Nyquist-Shannnon theorem [20].

Additionally, according to equation (2.18), expression of $\Delta k$ for a constant readout gradient is given by:

$$
\Delta k=\frac{\gamma G \Delta t}{2 \pi}
$$

where $\Delta t$ is the sampling interval (dwell time) along the gradient direction. The reciprocal of $\Delta t$ is called receiver bandwidth (BW):

$$
\mathrm{BW}=\frac{1}{\Delta t}
$$

Equation (2.24) indicates that the sample distance can be reduced either by increase sampling rate or by lower gradient strength. While the latter prolongs the total duration of the sampling, it will increase the signal-to-noise ratio (SNR). Therefore, a high value of the bandwidth corresponds to a short acquisition with low SNR, while a low value indicates a long readout time with high SNR [15, 21]. 


\subsection{Image Reconstruction}

\subsubsection{Gridding and FFT}

As mentioned above, for Cartesian sampling, all sampled $k$-space points lie in the Cartesian grid, the object image is then obtained by a direct application of FFT. In the case of non-Cartesian sampling (e.g., radial, spiral), the sampling points not necessarily lie on the Cartesian grid. Therefore, direct FFT does not apply. Nonuniform FFT (NUFFT) is employed instead to reconstruct images. This technique is also known as gridding and FFT in MRI. It contains the following steps:

- Density compensation. Since the sampling density is nonuniform in nonCartesian sampled $k$-space, a density compensation filter (DCF) is necessary to be employed. The DCF can be estimated by computing a Voronoi diagram or Dirichlet tessellation of the specific trajectory [22]. For radial sampling, a Ram-Lak Filter can be used:

$$
D_{\mathrm{RL}}(k)=\left\{\begin{array}{ll}
|\vec{k}| / n_{s} & |\vec{k}| \neq 0 \\
1 / 2 n_{s} & |\vec{k}|=0
\end{array} .\right.
$$

- Convolution and Inverse FFT. After density compensation, the resampling onto the Cartesian grid is achieved by the convolution of measured samples with the interpolation kernel. The ideal interpolation kernel is a proper scaled sinc function. Due to its unlimited support, the sinc kernel is not used in practice. Instead a radial Kaiser-Bessel kernel is usually employed

$$
K_{\mathrm{KB}}(d)= \begin{cases}\frac{1}{L} I_{0}\left(\beta \sqrt{1-(2 d / L)^{2}}\right) & |d| \leq \frac{L}{2} \\ 0 & |d|>0\end{cases}
$$

An inverse FFT is then applied on the interpolated $k$-space to get the image.

- Roll-off correction. Due to the convolution with a finite interpolation kernel, the obtained image exhibits a modulation with the Fourier transformation of the kernel, which is called roll-off effect. This can be removed by dividing the image with the Fourier transformation of the kernel. This process is referred to as roll-off correction. To compensate the second effect which is aliasing artifact in the image caused by the finite interpolation and rolloff correction, a two-times oversampling is usually employed. This helps to move the aliased side lobes away from the object. Image crop operation is then done in the end to get the final object image. 


\subsubsection{Iterative Reconstruction}

Image reconstruction can be also formulated as inverse problem. The equation for signal from one idealized single receiver coil (2.20) can be understood as the forward problem of MR image reconstruction:

$$
y(\vec{k})=P_{k} \mathcal{F} x(\vec{r})
$$

where $x$ is the unknown image, $y$ is the measured data, $\mathcal{F}$ is the continuous Fourier transform and $P_{k}$ is the trajectory defined by the restriction onto the measured kspace positions. After discretization, image $x$ and measured data $y$ can be replaced by a vector of image pixels $\boldsymbol{x}$ and signal samples $\boldsymbol{y}$ respectively. $\mathcal{F}$ then reduces to a matrix of Fourier coefficients $\mathcal{F}$ from a 2D Discrete Fourier Transformation (DFT). $P_{k}$ also can be represented by a matrix $\boldsymbol{P}$, which is diagonal in the case of Cartesian sampling. Combing all the linear operators, we have

$$
\boldsymbol{y}=\boldsymbol{A} \boldsymbol{x} \quad \text { with } \quad \boldsymbol{A}=\boldsymbol{P} \mathcal{F}
$$

representing the forward system matrix. For fully sampled Cartesian data, $\boldsymbol{A}$ is simply the matrix that applies the DFT to vector $\boldsymbol{x}$. In general, the equation cannot be inverted directly. Instead, the solution of the above formula can be defined as the minimizer of a functional, i.e.,

$$
\hat{\boldsymbol{x}}:=\underset{\boldsymbol{x}}{\operatorname{argmin}}\|\boldsymbol{A} \boldsymbol{x}-\boldsymbol{y}\|_{2}^{2}+\lambda \cdot R(\boldsymbol{x}) .
$$

The functional is composed of a least-squares data fidelity term as well as an additional regularization term $R$ and can then be solved iteratively by employing a number of numerical methods [23]. The formulation of reconstruction as inverse problem has several advantages in general: First, arbitrary sampling schemes (Cartesian or non-Cartesian) can easily be used. Second, the regularization term allows an incorporation of a prior knowledge of the unknowns. The above two properties enable the image being recovered from an incoherently undersampled dataset with proper sparsity regularizations (e.g., Wavelet or Total Variation (TV)) according to the compressed sensing theory [17, 18]. Finally, this formulation is easy to combine with multiple coils (i.e., parallel imaging).

\subsection{Parallel Imaging}

One limitation of MRI is the long acquisition time. Parallel imaging is a general technique to accelerate MRI using multiple phase-array coils [24] placed around 
the object (as seen in Figure 2.10). By exploiting additional spatial information from the coil sensitivity profiles, it is possible to compensate the missing $k$-space data due to undersampling. Methods for parallel imaging can be divided into two categories, i.e., image space and $k$-space based methods respectively. The clinical applications of parallel imaging starts from SMASH (simultaneous acquisition of spatial harmonics) [25] and SENSE (sensitivity encoding) [26] algorithms introduced in late 1990s. SMASH is a kind of $k$-space based method, while SENSE belongs to the image-space based methods. Since then, both classes of method have been further improved to utilize the $k$-space data more efficiently [27-31]. A comparison of different approaches can be found in [32-34].

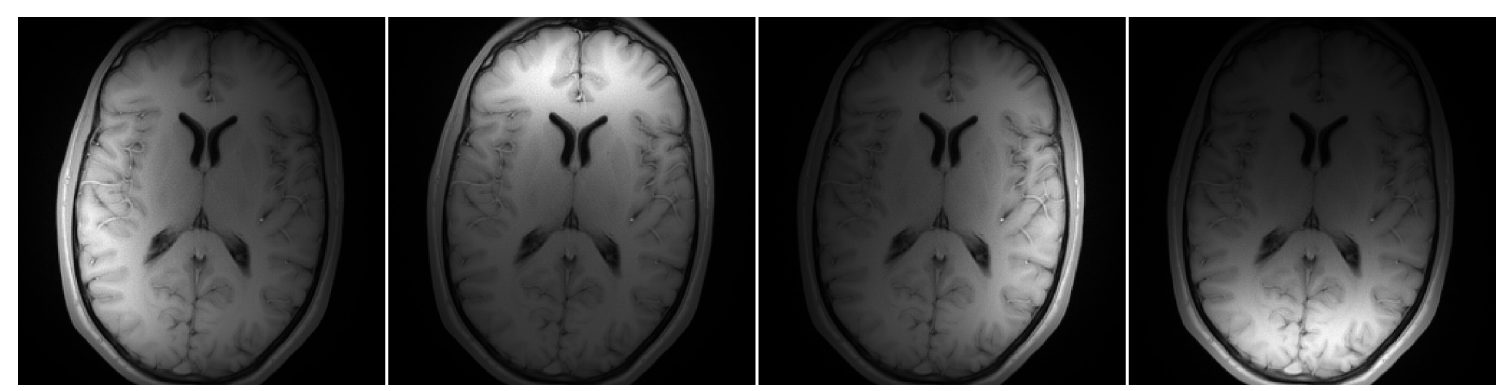

Figure 2.10: Four individual images each calculated from the signal of its respective receive coil exhibit different spatial sensitivity profiles.

The MRI signal obtained from multiple receiver coils is given by:

$$
s_{j}(t)=\int \rho(\vec{r}) c_{j}(\vec{r}) e^{-i 2 \pi \vec{k}(t) \cdot \vec{r}} d \vec{r}+n(t) \quad j=1, \ldots, N
$$

where $\rho$ is the proton density, $c_{j}$ is the complex-valued spatial sensitivity profile of individual receive coil, and $\vec{k}(t)$ is the chosen trajectory. Signal $s_{j}$ is disturbed by noise $n$. After discretization, this process can be considered mathematically as mapping $\rho$ and $c_{j}$ to the sampled $k$-space data $y$ with an operator $F$ :

$$
y=F(x) \quad \text { with } \quad F: x \mapsto\left(\begin{array}{c}
P_{k} \mathcal{F}\left(\rho \cdot c_{1}\right) \\
\vdots \\
P_{k} \mathcal{F}\left(\rho \cdot c_{N}\right)
\end{array}\right)
$$

where $P_{k}$ is the projection onto the trajectory, $\mathcal{F}$ is Fourier transformation, $x$ is the unknown variable. The availability of prior knowledge also determines whether the reconstruction problem will become linear of nonlinear. The forward and inversion model is demonstrated in Figure 2.11.

In SENSE based algorithms, the coil sensitivities are precalculated, mostly using the autocalibration signal (ACS) line methods. For ACS based methods, a small region in the center of $k$-space is sampled at full Nyquist rate. The fully sampled 


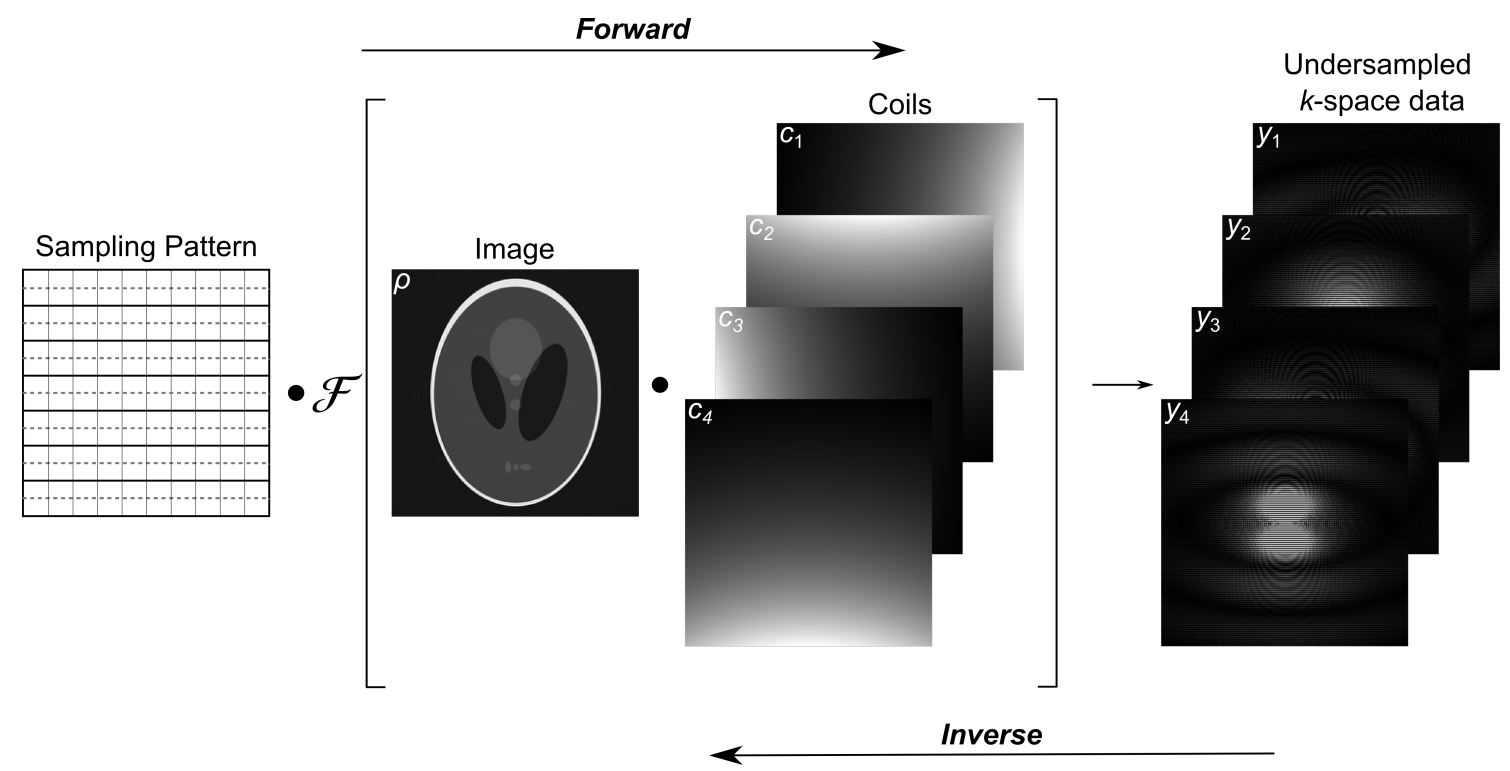

Figure 2.11: Scheme illustration of forward and inverse model in parallel imaging. The forward model maps the Fourier transform $(\mathcal{F})$ of the dot product of image $\rho$ and coil sensitivities $c_{j}$ evaluated on the sampling trajectory to the $k$-space data $y_{j}$ (with two fold Cartesian undersampling). The inverse model is then to estimate image and/or coil sensitivities from the undersampled data.

regions are then filtered and inversely Fourier transformed to create low-resolution images for each coil element. Coil profiles are then taken from low-order polynomials, fitted to the individual images [35]. After estimation of coil sensitivities, the reconstruction of proton density image can be considered as an inverse problem, which can be solved by minimizing the following cost function:

$$
\rho:=\underset{\rho}{\operatorname{argmin}} \sum_{j=1}^{N}\left\|P_{k} \mathcal{F}\left(\rho \cdot c_{j}\right)-y_{j}\right\|_{2}^{2}+\lambda \cdot R(\rho)
$$

with $R(\rho)$ the regularization term for the proton density image. $\lambda$ is the regularization parameter controlling the balance of noise reduction and the preservation of image details. The above formula (2.33) is an extension of equation (2.30) with multiple coils. When a quadratic regularization is used, this problem can then be solved with very efficient algorithms such as the conjugate gradient (CG) method which is known as CG-SENSE. Other non-smooth regularizations such as TV or Wavelet can also be employed. With proper incoherent sampling scheme (e.g., variable-density Poisson-disc sampling or radial trajectories), the combination of parallel imaging and compressed sensing can achieve a higher acceleration factor than individually using CS or parallel imaging [18, 36-38].

On the other hand, $k$-space based parallel imaging algorithms, like SMASH and GRAPPA (generalized autocalibrating partially parallel acquisitions) [29], try to 
complete the missing data in $k$-space before reconstructing the image using FFT. These algorithms are all based on the $k$-space locality principle [39], which assumes that points nearby in $k$-space are strongly correlated. Therefore, missing samples can be approximated as a linear combination of nearby $k$-space samples from all coils. After recovery of missing data from each channel, the coil image can be reconstructed by FFT and followed by employing coil combination methods such as the root of sum of squares (RSS) method. More information about $k$-space based parallel imaging see [34].

There are several ways to combine data from different coils in order to obtain a single reconstructed image. The best unbiased estimation for the image is given by [24, 40]:

$$
\rho_{\mathrm{est}}=\frac{1}{\sum_{j}\left|c_{j}\right|^{2}} \sum_{j} c_{j}^{*} \hat{s}_{j}
$$

with an assumption that the signal noise is independently and identically distributed (i.i.d) Gaussian white noise. Here $c_{j}^{*}$ is the complex conjugate of the coil sensitivities and $\hat{s}_{j}$ is the image for each individual coil. Since the knowledge of coil sensitivities is required, a RSS operation is often used instead:

$$
\rho_{\mathrm{rss}}=\sqrt{\sum_{j}\left|\hat{s}_{j}\right|^{2}}
$$

The above equation (2.35) is an approximation of formula (2.34), where the sensitivity is approximated by $c_{j}^{*}=\hat{s}_{j}^{*}$. Noteworthy, this assumption is only valid for locations with high signal. In the region of low signal intensity, a bias will be introduced [41, 42]. Alternatively, a usage of the more complicated phase-preserving method introduced in [40] will help to avoid this problem.

\subsection{MRI System}

All studies in this work were conducted on a Siemens Prisma whole body human scanner (Siemens AG, Erlangen, Germany) with the main magnetic field $B_{0}=$ 2.89 Tesla ( $\mathrm{T}$ ) and two build-in body coils for RF excitation and signal receiving. The gradient system has a maximum gradient strength of $80 \mathrm{mT} \mathrm{m}^{-1}$. The raster time for gradient switching is $10 \mu \mathrm{s}$ and the maximal slew rate is $200 \mathrm{mT} \mathrm{m}^{-1}$ $\mathrm{ms}^{-1}$. Several receiver coils are provided by the system for signal detection, i.e., a 64-channel head coil, a 18-element thorax coil and the 32-element spine coil. Additionally, a commercial reference phantom (Diagnostic Sonar LTD, Scotland, UK) consisting of 6 compartments with defined $T_{1}$ values surrounded by water is used for quantitative validations in this study (Figure 2.12). 


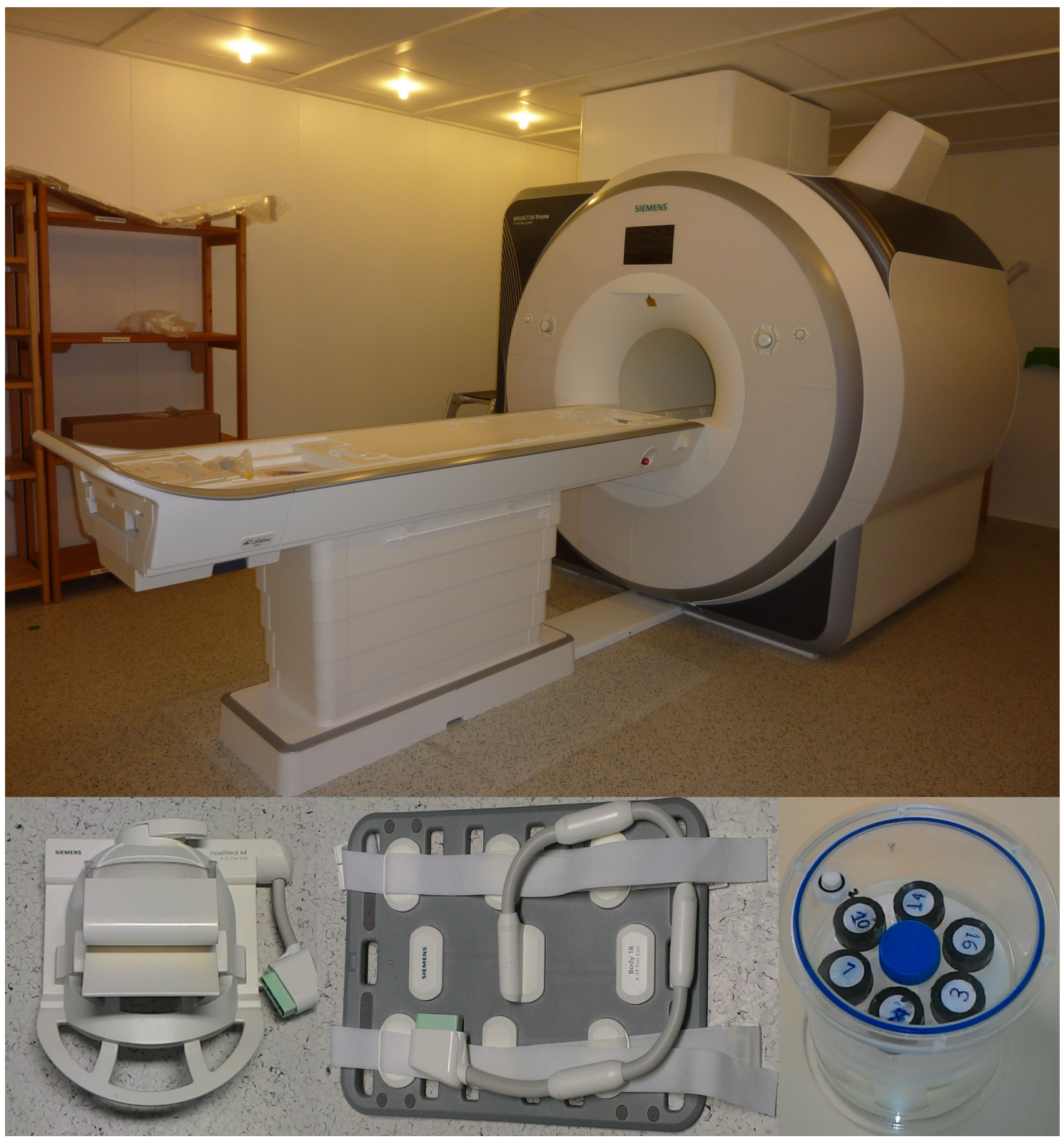

Figure 2.12: (Top) MRI system, (bottom left) 64-channel head coil, (bottom middle) 18-element thorax coil and (bottom right) a commercial $T_{1}$ phantom used in this thesis. 


\section{Real-time MRI}

Real-time MR imaging refers to continuous monitoring of moving objects, such as the human cardiovascular system in real time. To achieve this, specific strategies for image acquisition and reconstruction have to be employed. Earlier applications are based on so-called single-shot gradient-echo sequences, such as echoplanar imaging [43] and spiral imaging [44]. Since they usually require a long readout, images are prone to artifacts caused by off-resonance effects. Instead, a recent developed MRI technique which employs radial MRI and regularized nonlinear inversion does not have this problem and can achieve a much higher spatial-temporal resolution. The chapter introduces the general acquisition and reconstruction methods for this technique.

\subsection{Undersampled Radial FLASH}

The FLASH pulse sequence is a gradient-echo sequence with low flip angle RF pulses and short repetition time [9, 45]. Because low flip angle excitation is used instead of $90^{\circ}$ pulse in spin echo sequences, most of the longitudinal magnetization is remained and an immediate next excitation is then allowed. With a rapid repetition of this sequence, fast imaging is therefore possible. Depending on the response of the transverse magnetization in steady state, there are three types of sequences in the generic FLASH technique, i.e., spoiled, refocused FLASH and balanced steady state free precession (bSSFP). The radial FLASH sequence diagram is shown in Figure 3.1(a). Spoiled FLASH employs RF spoiling or gradient spoiling to destroy the transverse magnetization. For radial spoiled FLASH, besides RF spoiling with random phase increment, no additional spoiler gradient is necessary as the gradient spoiling is achieved through the applications of varying readout gradients from TR to TR. Spoiled FLASH produces images with $T_{1}$ contrast and has been used primarily for real-time MRI applications ranging from speaking, cardiovascular function to quantitative phase-contrast MRI. On the contrary, in refocused FLASH a gradient of the same length but of opposite polarity is applied after readout to keep the residual transverse magnetization. The net phase per TR is constant. Refocused FLASH offers a $T_{2} / T_{1}$ contrast and has been used for the study of temporomandibular joint (TMJ) dysfunctions. Further, to realize a bal- 
(a)
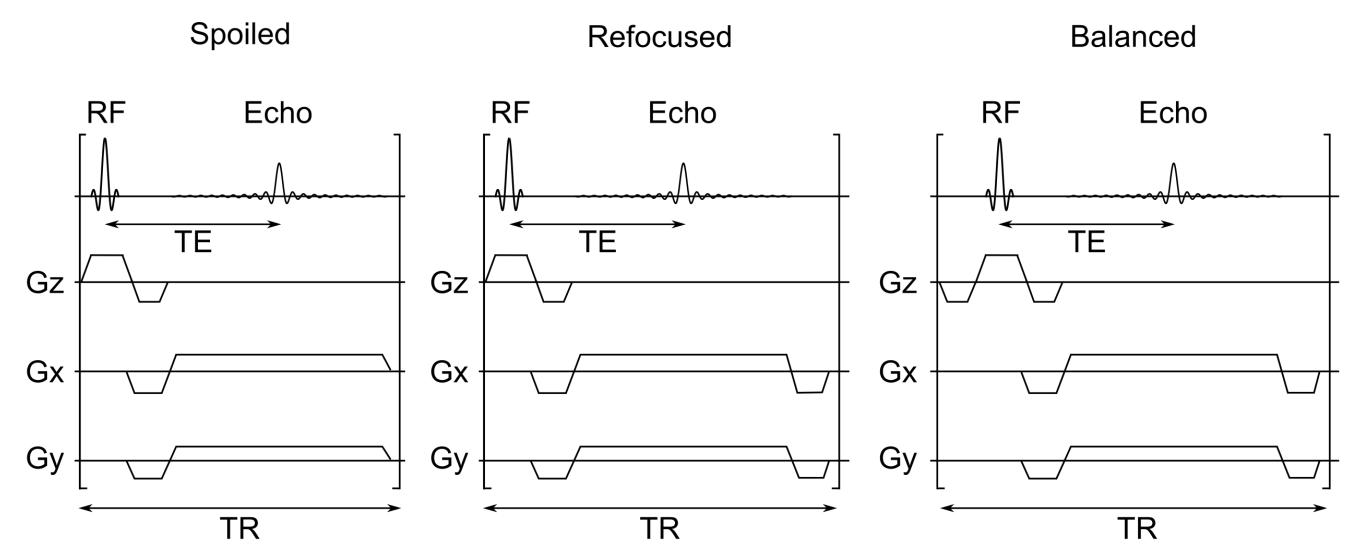

(b)
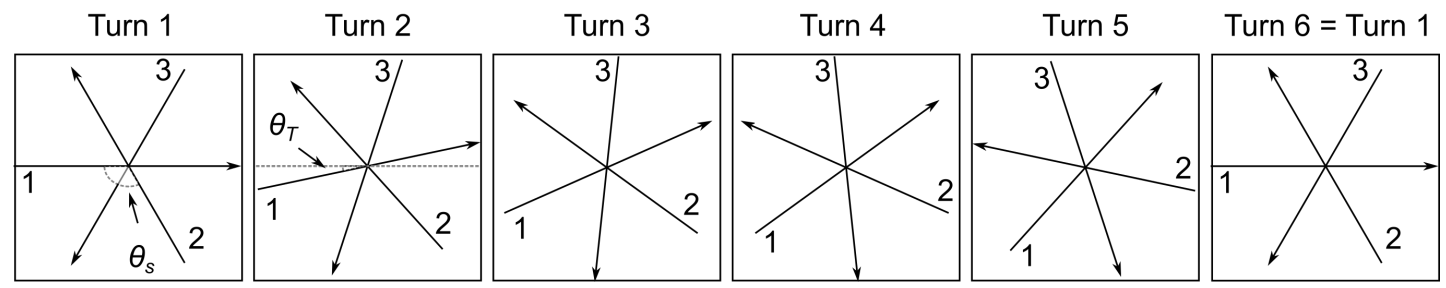

Figure 3.1: Undersampled radial FLASH sequences. (a) Sequence diagram of spoiled, refocused and balanced radial FLASH techniques. (b) An example of undersampled radial spoke distribution in real-time MRI (i.e., 3 spokes per frame with 5 sequential turns).

anced condition which has zero net phase per TR, a dephasing gradient along the slice selection direction with the same strength and direction as the slice selection refocusing gradient is applied before each excitation. The bSSFP sequence offers excellent $T_{2} / T_{1}$ contrast between flowing blood and heart muscle (myocardium), therefore it is widely used in cardiovascular magnetic resonance imaging (CMR) studies. One main drawback of the bSSFP sequence is that it is prone to banding artifacts due to off-resonance effects.

Radial sampling was proposed in the beginning of MRI and regained a lot of interest in the last decade. Radial trajectories offer several advantages over the traditional Cartesian scheme. First, due to the absence of phase encoding and oversampling of $k$-space center, radial is less sensitive to motion which often induces ghosting artifacts in Cartesian sampling. Second, radial sampling is quite tolerant to undersampling. Most object information remains visible even for significant undersampling factors. This is due to the fact that the center of $k$-space is always densely sampled and most of the energy is still kept in the case of undersampling. Third, a readout oversampling which enlarges the circular-supported FOV and therefore helps to remove the aliasing effects can be easily employed without increasing acquisition time. Finally, each spoke carries an equal amount of low and high spatial frequencies, offering a more homogeneous image update in dynamic MRI. As a result of these properties, radial sampling is favorable for 
real-time MRI applications [21].

Radial spokes are acquired in a certain view order to fill the $k$-space. In realtime MRI, the spokes are uniformly distributed in one image (turn) and then are sequentially rotated between consecutive images (turns). The sequential reordering scheme is repeated every $N_{T}$ (e.g., $N_{T}=5$ ) images (turns) as shown in Figure 3.1 (b). Both $N_{T}$ and the number of spokes per frame $\left(N_{s}\right)$ is chosen to be odd integers as a consequence of the total angle coverage set to be $2 \pi$ [21]. Therefore, the angle increment between two successive spokes $\theta_{s}$ is

$$
\theta_{s}=\frac{2 \pi}{N_{s}}
$$

and the angular increment between two consecutive turns $\theta_{T}$ is

$$
\theta_{T}=\frac{2 \pi}{\left(N_{s} \cdot N_{T}\right)}
$$

\subsection{Parallel Imaging as Nonlinear Inverse Problem}

As mentioned in chapter 2, reconstruction methods for autocalibrated parallel imaging typically consist of two steps: determination of coil sensitivities from reference lines followed by linear reconstruction of image content. This process is suboptimal because only a subset of data is used to determine the coil profiles [34]. Therefore, methods that jointly estimate image content and coil sensitivities from the whole available data have been proposed. The first method of this kind is joint SENSE (JSENSE) [46]. With the help of alternating minimization method, image content and coil sensitivities are updated alternatively, both of them are optimized until a joint solution is found. By extending this idea, a more general framework for solving this problem was introduced by formulating parallel imaging as a nonlinear inverse problem [47].

\subsubsection{Algorithms}

The MRI signal equation is understood as a nonlinear operator equation with an operator $F$ which maps image content $\rho$ and coil sensitivity profiles $\left(c_{1}, \ldots, c_{N}\right)^{T}$ to the measured data $y$ :

$$
y=F(x) \text { with } \quad x=\left(\begin{array}{c}
\rho \\
c_{1} \\
\vdots \\
c_{N}
\end{array}\right) \quad \text { and } F: x \mapsto\left(\begin{array}{c}
P_{k} \mathcal{F}\left(\rho \cdot c_{1}\right) \\
\vdots \\
P_{k} \mathcal{F}\left(\rho \cdot c_{N}\right)
\end{array}\right)
$$


where $\mathcal{F}$ is the Fourier transformation, $P_{k}$ is the projection onto the trajectory. The above nonlinear equation is then solved by the iteratively regularized GaussNewton method ( $\overline{\text { IRGNM }}$, , for more details see [48]. In general, this method first linearizes $F(x)$ at $x_{n}$ with $F\left(x_{n}+d x\right) \approx D F\left(x_{n}\right) d x+F\left(x_{n}\right)$, where $n$ is the $n$th Gauss-Newton step. $d x$ is then updated by minimizing the following regularized problem:

$$
\left\|D F\left(x_{n}\right) d x-\left(y-F\left(x_{n}\right)\right)\right\|_{2}^{2}+\alpha_{n}\left\|x_{n}+d x-x_{0}\right\|_{2}^{2}
$$

where the regularization parameter $\alpha_{n}$ is chosen to be of the form $\alpha_{n}=\alpha_{0} q^{n}$ with $q \in(0,1)$. The update $d x$ has an explicit expression:

$$
d x=\left(D F\left(x_{n}\right)^{H} D F\left(x_{n}\right)+\alpha_{n} I\right)^{-1}\left(D F\left(x_{n}\right)^{H}\left(y-F\left(x_{n}\right)\right)+\alpha_{n}\left(x_{n}-x_{0}\right)\right)
$$

which can be solved using very efficient methods, such as the $\mathrm{CG}$ algorithm. Under certain conditions the iterative updates of $x_{n+1}=x_{n}+d x$ converge to a solution.

However, a direct application of IRGNM to the above nonlinear problem would yield an unrealistic solution because the equation is highly underdetermined even for the fully sampled case. This problem is overcome by adding an additional regularization term, which penalizes high frequencies in the spectrum of the coil profiles in order to enforce the intrinsic smoothness of coils. Therefore, the operator and the representation of the coil profiles are transformed with a preconditioning matrix $W$ which contains weighted Fourier coefficients of the coil profiles:

$$
\left(\begin{array}{c}
\rho \\
\hat{c}_{1} \\
\vdots \\
\hat{c}_{N}
\end{array}\right)=\left(\begin{array}{cccc}
I & & & \\
& \left(1+s\|\vec{k}\|^{2}\right)^{l / 2} \mathcal{F} & & \\
& & \ddots & \\
& & & \left(1+s\|\vec{k}\|^{2}\right)^{l / 2} \mathcal{F}
\end{array}\right)\left(\begin{array}{c}
\rho \\
c_{1} \\
\vdots \\
c_{N}
\end{array}\right)
$$

with $I$ the identity, $\|\vec{k}\|$ the distance to $k$-space center, and $s$ and $l$ empirically chosen weighting parameters. The IRGNM is then applied to the transformed but equivalent system of equations:

$$
\begin{aligned}
\hat{x} & =W^{-1} x \\
G \hat{x} & =F W \hat{x}=y
\end{aligned}
$$

such that the unknown $\hat{c}_{j}$ is defined in the frequency domain. The above nonlinear inversion (NLINV) method was proposed for Cartesian MRI initially. To allow for real-time MRI, NLINV is extended to radial sampling by choosing $P_{k}$ to be the projection onto a radial trajectory.

Further, to exploit the prior knowledge that adjacent images have similar con- 
tent, the algorithm is modified to include the temporal regularization with respect to the previous frame:

$$
\alpha_{n}\left\|x_{n}+d x-\beta \cdot x_{\mathrm{prev}}\right\|_{2}^{2}
$$

where $x_{\text {prev }}$ is the previously reconstructed image and $\beta$ is the damping factor which is chosen between 0.7 and 0.9 depending on application [49].

To achieve reasonable reconstruction times, the above iterative algorithms are implemented on multiple graphic processing units (GPU) $[50,51]$ and fully integrated into the reconstruction pipeline of the MRI system.

\subsubsection{Preprocessing}

Prior to NLINV reconstruction, the raw data from multiple coils is firstly corrected for gradient delay errors [52] and then compressed to 10 virtual channels using principle component analysis (PCA). For radial encoding, a convolution based gridding [53] without density compensation is used to move the interpolation into the Cartesian grid. The interpolated data is then normalized such that the $L^{2}$ norm of the data matrix is 100 .

\subsubsection{Postprocessing}

After completion of the reconstruction process, a temporal median filter with a length of the number of turns can be used to remove the residual reconstruction artifacts (streaking) that differ from frame to frame. Further, a modified non-local means denoising algorithm has been developed for spatial filtering [54]. Noteworthy, for quantitative $T_{1}$ mapping, no median filter is used to avoid corruption of the recovery curve which would otherwise result in incorrect $T_{1}$ values. 



\section{Introduction to Quantitative T1 Mapping Techniques}

\subsection{Introduction}

Rapid mapping of the spin-lattice relaxation process with quantitative evaluations of $T_{1}$ relaxation times is of utmost interest in many clinical applications. $T_{1}$ values of myocardial tissue before and after contrast uptake can be used to detect and access various cardiomyopathies. Further, pathology in brain tissue is well reflected in abnormal $T_{1}$ maps, for more applications see [55]. Several methods have been proposed for $T_{1}$ mapping in MRI studies [56, 57]. The following chapter reviews the three most commonly used methods, namely inversion recovery (IR), IR LookLocker ( $(\mathrm{LL})$ and variable flip angle (VFA) based methods. A sequence diagram for the three methods is given in Figure 4.1 .

\subsection{Inversion Recovery T1 mapping}

The IR method is referred to as the gold standard method. This technique originates from the NMR experiments performed in the late 1940s [58, 59]. It comprises inverting the longitudinal magnetization $M_{z}$ and sampling it as it recovers towards equilibrium. The process is described by the Bloch equation

$$
\frac{d M_{z}(t)}{d t}=\frac{M_{0}-M_{z}(t)}{T_{1}} .
$$

There are two main RF pulses in this sequence. First, a $180^{\circ}$ inversion pulse inverts the initial magnetization. $M_{z}$ will then recover to the equilibrium longitudinal magnetization $M_{0}$ with relaxation time $T_{1}$. After an inversion time TI, the second pulse tips the recovered longitudinal magnetization into the transverse plane for measurement. The transversal signal equation of this process at different TI can then be expressed as

$$
S(\mathrm{TI})=S_{0}\left(1-2 \exp \left(-\mathrm{TI} / T_{1}\right)\right)
$$


with $S_{0}$ a complex signal acquired from the equilibrium longitudinal magnetization $M_{0}$. A nonlinear least squares fitting can then be performed to get $T_{1}$ values. The above equation (4.2) assumes a perfect inversion pulse which is rarely the case in practice and TR $\gg T_{1}$ is also needed. This can be overcome by fitting the signal to a more general model proposed in [60]

$$
S(\mathrm{TI})=a+b \exp \left(-\mathrm{TI} / T_{1}\right)
$$

where both $a$ and $b$ are complex variables. This model takes both inversion efficiency and insufficient recovery into consideration. Therefore, it is more accurate and the waiting for the magnetization to full recovery within one TR is not necessary. However, as the number of unknown parameters for fitting is increased, the SNR of the final $T_{1}$ map will then be compromised [61].

The general limitation of the IR method is that it is really inefficient because only one line of $k$-space data is sampled during inversion recovery, the next inversion pulse is applied only after a long longitudinal recovery period. Although methods like [60] explained above have been proposed to shorten the waiting time, the total data acquisition time is still extremely long. One way to overcome this problem is to sample the longitudinal magnetization continuously during the recovery period, which leads to the Look-Locker based method.

(a)

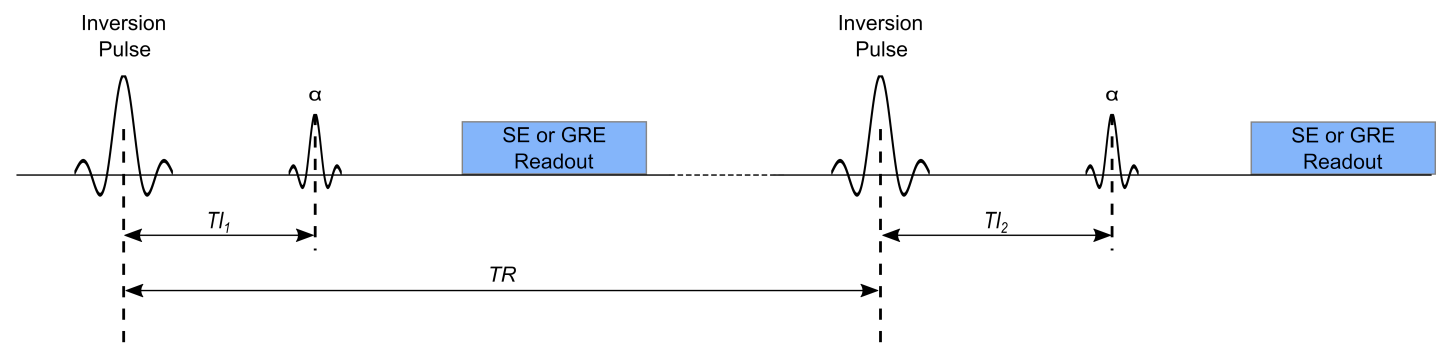

(b)

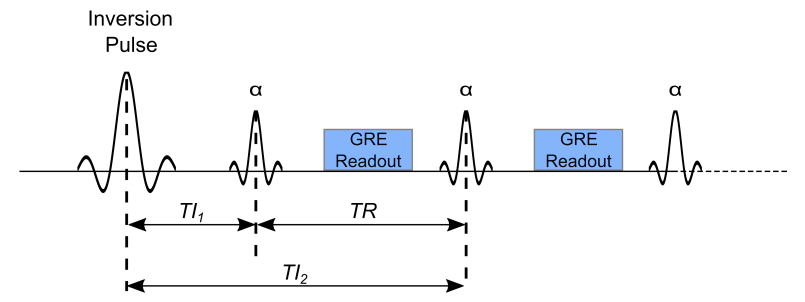

(c)

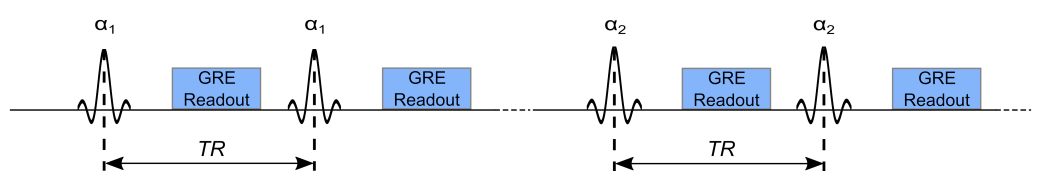

Figure 4.1: $T_{1}$ mapping sequence diagram. (a) Inversion Recovery (b) Inversion Recovery Look-Locker (c) Variable Flip Angle. 


\subsection{Inversion Recovery Look-Locker T1 mapping}

The Look-Locker sequence [62] comprises a magnetization preparation pulse, i.e., inversion or saturation pulse, followed by a series of low flip angle RF excitations with spoiled gradient echo (FLASH) readout (Figure 4.2). By continuously sampling multiple time points along the recovery curve, the Look-Locker method is the most time efficient method for $T_{1}$ mapping [63]. The longitudinal magnetization of this process can be described by

$$
M(t)=M_{\mathrm{ss}}-\left(M_{0}+M_{\mathrm{ss}}\right) \exp \left(-t / T_{1}^{*}\right)
$$

with $M_{\mathrm{ss}}$ the steady state magnetization, $M_{0}$ the equilibrium magnetization and $T_{1}^{*}$ the effective relaxation time given by

$$
\frac{1}{T_{1}^{*}}=\frac{1}{T_{1}}-\frac{\ln [\cos (\alpha)]}{\mathrm{TR}}
$$

where TR is the repetition time and $\alpha$ is the flip angle. Since knowing the exact flip angle $\alpha$ is impossible, the direct computation of $T_{1}$ from equation (4.5) is difficult. Instead, with the three parameters $M_{\mathrm{ss}}, M_{0}, T_{1}^{*}$ obtained from equation (4.4) and the assumption $\mathrm{TR} \ll T_{1}^{*}, T_{1}$, the desired $T_{1}$ then can be calculated according to

$$
T_{1}=\frac{M_{0}}{M_{\mathrm{ss}}} \cdot T_{1}^{*}
$$

Noteworthy, the above formula holds true only for small flip angles $\alpha$ in FLASH readout. Otherwise, the recovery curve will be more disturbed and therefore the contrast by $T_{1}$ difference will vanish for higher flip angles. In addition, the spoiling of the transverse magnetization will become more difficult. It is recommended that the flip angle should be less or equal than $10^{\circ}$ [64].

Although the signal model is derived for spoiled gradient echo readout, other readouts have also been proposed in combination with Look-Locker concept for fast $T_{1}$ mapping, such as EPI [65] and TrueFISP [66]. The main advantage of EPI readout is its speed, a whole image is produced from a single readout train. On the other hand, the EPI readout is very sensitive to field inhomogeneities which will cause image distortions in the final $T_{1}$ maps. For TrueFISP readout, the recovery curve is less perturbed, provided the tissue does not have a short $T_{2}$. Then the relaxation rate $T_{1}$ can be approximated by $T_{1}^{*}$. The problem of this method is that it is very sensitive to the off-resonance effect, which will introduce typical banding artifacts in the final $T_{1}$ maps especially at high field strength (e.g., 3T or higher).

To avoid these artifacts, the main focus of this thesis is to develop fast IR LL $T_{1}$ mapping methods based on FLASH readout. 


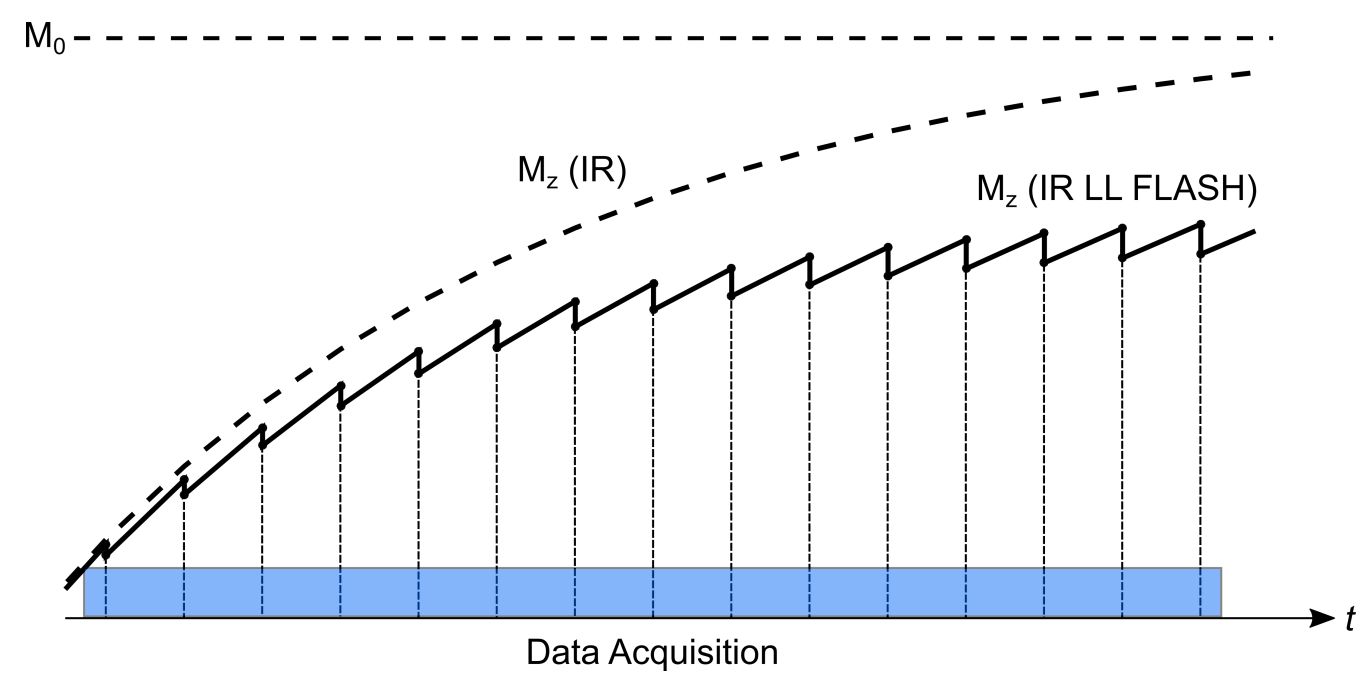

Figure 4.2: The longitudinal magnetization curve of IR (dashed line) and that of IR LL sequence with FLASH readout (solid line). $M_{0}$ is the equilibrium magnetization, $M_{z}$ is the longitudinal magnetization.

\subsection{Variable Flip Angle T1 mapping}

The VFA method is another fast $T_{1}$ mapping technique which allows to acquire 3D $T_{1}$ maps in clinically feasible times [67, 68]. This requires an acquisition of two or more spoiled gradient-echo measurements with constant TR, TE but different flip angles. The steady state signal of a FLASH sequence as a function of the flip angle $\alpha$ is given by:

$$
S(\alpha)=M_{0} \sin (\alpha) \frac{1-e^{-\mathrm{TR} / T_{1}}}{1-\cos (\alpha) e^{-\mathrm{TR} / T_{1}}} e^{-\mathrm{TE} / T_{2}^{*}} .
$$

Since TR, TE are fixed, both proton density $M_{0}$ and $T_{1}$ can be determined from the signals measured at multiple flip angles by performing a nonlinear least-square fit to the measured values of $S(\alpha)$ as a function of $\alpha$.

By reordering equation. (4.7), an easier-to-implement expression can be derived, i.e.,

$$
\frac{S(\alpha)}{\sin (\alpha)}=\frac{S(\alpha)}{\tan (\alpha)} e^{-\mathrm{TR} / T_{1}}+M_{0}\left(1-e^{-\mathrm{TR} / T_{1}}\right) e^{-\mathrm{TE} / T_{2}^{*}} .
$$

The above equation can be considered as a transformation of the points $(\alpha, S(\alpha))$ into a plane where the coordinate pairs are

$$
(x(\alpha), y(\alpha))=\left(\frac{S(\alpha)}{\tan (\alpha)}, \frac{S(\alpha)}{\sin (\alpha)}\right)
$$

with slope $e^{-\mathrm{TR} / T_{1}}$ and ordinate intercept $M_{0}\left(1-e^{-\mathrm{TR} / T_{1}}\right) e^{-\mathrm{TE} / T_{2}^{*}}$. The slope of the straight line and with it $T_{1}$ can be estimated by the linear least squares method. Figure 4.3 demonstrates $T_{1}$ estimation curve by equation (4.7) and equation (4.8) of simulated FLASH signals acquired with 10 different flip angles at a fixed TR of 
$5 \mathrm{~ms}$ [69].
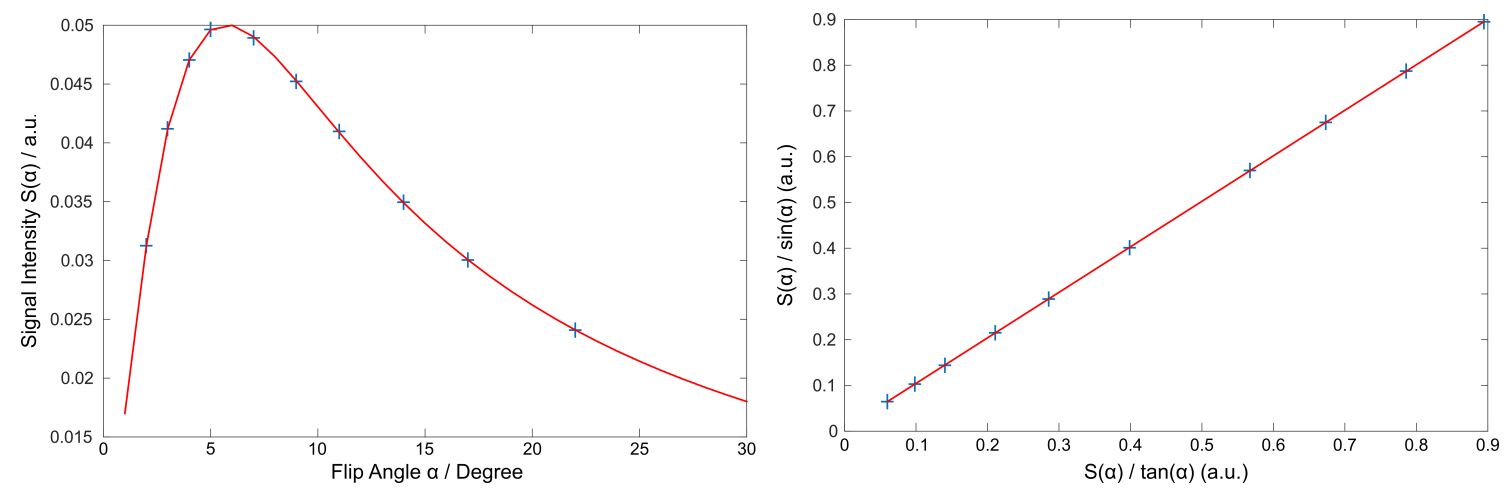

Figure 4.3: $T_{1}$ estimation by two different models in VFA method using ten flip angles [69]. (Left) The result of the original signal fitted to equation (4.7), (right) the fitting result of the transformed signal to the linear equation (4.8). The flip angles are assumed to be perfectly known.

In principle, two images from different flip angle readouts will be enough to determine the $T_{1}$ values, which makes the VFA method very attractive. However, this method normally requires an exact knowledge of the flip angle $\alpha$ for every voxel in the FOV. In practice, the $B_{1}$ pulse amplitude varies across FOV, which will result in artifacts as spatial variations in the final $T_{1}$ maps. This problem is more dominant at field strength of 3T or higher. Therefore, a flip angle $\left(B_{1}\right)$ mapping is essential. Several $B_{1}$ mapping techniques can be employed [70-72]. However, this additional step limits the practical applications of VFA method. 



\section{Single-shot Inversion-recovery Look-Locker T1 Mapping by Real-time MRI}

\subsection{Introduction}

The inversion-recovery Look-Locker method (see chapter 4) is the most time efficient technique for $T_{1}$ mapping [63]. However, to achieve a spatial and temporal resolution that is sufficient for clinical use, segmented acquisitions instead of a single inversion preparation are usually required. To overcome the problem of long data acquisition time caused by the segmented acquisitions, several techniques have been developed. One efficient way for acceleration is parallel imaging. By taking complementary information from multi-coil arrays, acceleration factors of 2-4 could be achieved. Radial sampling is another way for speed-up [73, 74]. With a combination of sliding-window based image reconstruction [75], multiple images with different contrasts can be generated within one single inversion recovery and single-shot $T_{1}$ mapping is allowed. However, with increasing spatial resolution, larger window sizes are needed for reconstruction which will compromise the temporal resolution and affect the $T_{1}$ accuracy. Additionally, a model-based approach which estimates parameter maps directly from undersampled $k$-space data has been proposed [76]. These kind of methods bypass the two steps of image reconstruction and pixel-wise fitting and do not need to compromise between image quality and temporal resolution. However, only a limited spatial resolution has been achieved so far [76].

In this chapter, a single-shot high-resolution IR LL $T_{1}$ mapping method is proposed by taking advantage of the real-time MRI techniques based on highly undersampled radial FLASH acquisitions and iterative image reconstruction. The proposed approach covers the spin-lattice relaxation process after (nonselective) inversion of the longitudinal magnetization by a series of images reconstructed from highly undersampled data. Several acquisition and reconstruction parameters of the proposed method are optimized and validated on numerical simulation, experimental phantom and in vivo studies. A recent proposed non-local means 
(NLM) filter is further applied to the reconstructed images prior to pixel-wise fitting for precision improvement. Finally, an extension of the proposed method to single-shot multi-slice $T_{1}$ mapping is presented.

\subsection{Materials and Methods}

\subsubsection{Single-shot IR LL Radial FLASH}

The single-shot IR LL radial FLASH sequence comprises an initial IR module which consists of a nonselective adiabatic $180^{\circ} \mathrm{RF}$ pulse with a spoiler gradient and followed by a continuous slice-selective radial FLASH readout that monitors the $T_{1}$ relaxation recovery with complementary sets of spokes each covering $360^{\circ}$. Typically, 5 sequential frames of the same slice are acquired with spatially distinct radial spokes which interleave spokes of preceding sets as previously described [77]. Spoiling of residual transverse magnetizations are accomplished by random $\mathrm{RF}$ phases [78]. The diagram of this sequence is shown in Figure 5.1 and the distribution of the spokes can be found in Figure 3.1(b).

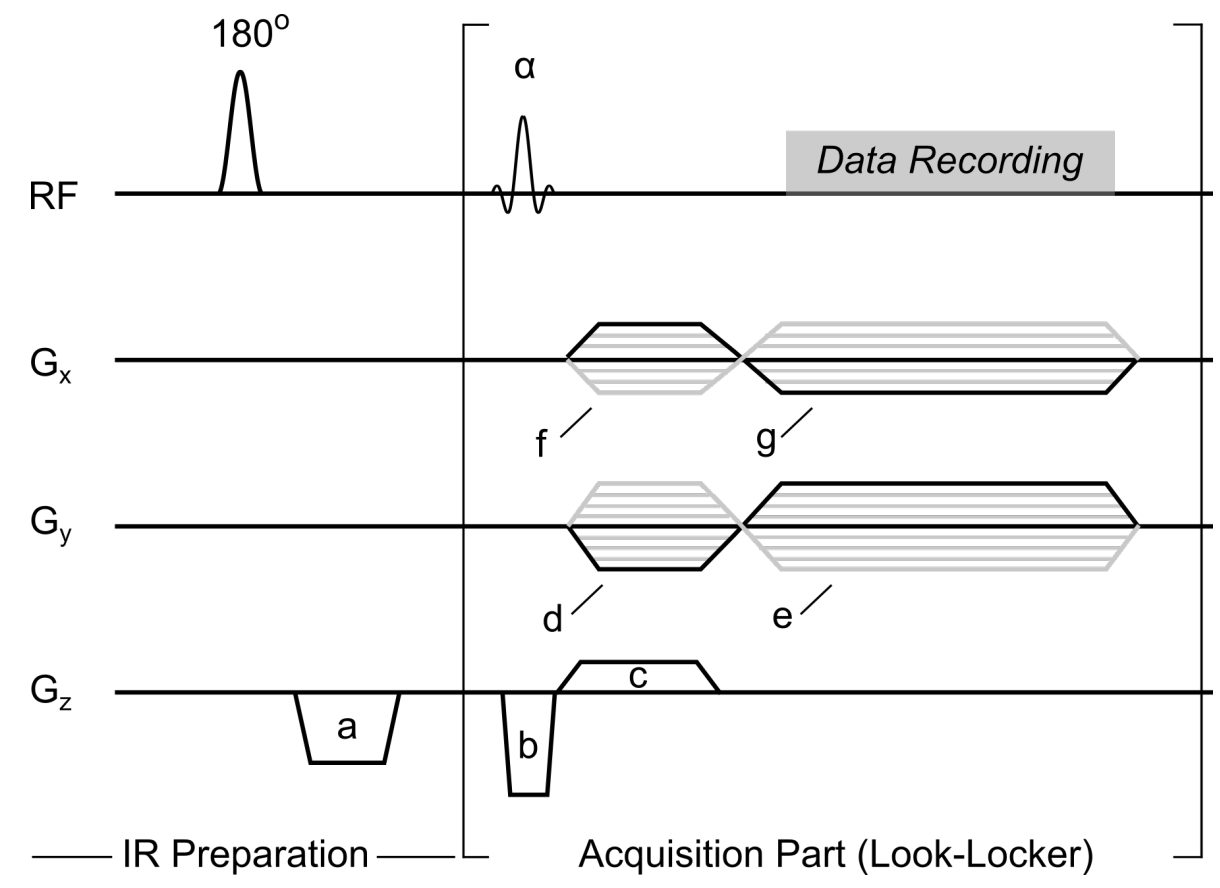

Figure 5.1: Schematic diagram for single-shot inversion recovery Look-Locker radial FLASH MRI. The acquisition part is repeated during inversion recovery. Gradient amplitudes vary for different repetitions (gray lines). (a) Spoiler, (b) slice-selection, (c) rephasing, (d,f) prephasing, (g,e) readout. The $180^{\circ}$ pulse is a non-selective adiabatic inversion pulse (Hyperbolic secant pulse). 


\subsubsection{Image Reconstruction}

The image reconstruction part is based on the NLINV algorithm as introduced in chapter 3. For $T_{1}$ mapping, the reconstruction has been modified and separated into two steps. First, the coil sensitivities are estimated by NLINV from a few frames starting from the relaxation process close to full recovery and proceeding toward the initial inversion. Because this strategy starts with only very mild changes from frame to frame, it helps to achieve high-quality reconstruction of images and coil profiles. In fact, coil sensitivities are estimated from 10 last frames corresponding to 2 times 5 frames with complementary radial encodings. Subsequently, the estimated coil sensitivities are fixed and used for reconstruction of the entire data set. The reconstruction of the individual image then becomes a linear problem:

$$
\hat{x}=\underset{x}{\operatorname{argmin}}\|F(x)-y\|_{2}^{2}+\alpha\left\|x-\beta \cdot x_{\text {prev }}\right\|_{2}^{2} .
$$

The linear forward operator $F$ is given by:

$$
F: x \mapsto\left(\begin{array}{c}
P \mathcal{F}\left\{c_{1} \cdot x\right\} \\
\vdots \\
P \mathcal{F}\left\{c_{N} \cdot x\right\}
\end{array}\right),
$$

where $\mathcal{F}$ is the Fourier transformation, $P$ is the orthogonal projection onto the trajectory, $c_{j}$ is the estimated $j$ th coil sensitivity and $x$ is the current image to be estimated, $x_{\text {prev }}$ is the previously reconstructed frame used for temporal regularization as in real-time MRI, $\alpha$ is the regularization parameter and $\beta$ is the damping factor which controls information from previous frames in the $L^{2}$ regularization term. The above linear problem is then solved by the linear version of the NLINV algorithm [47] in a time-reversed order. For the choice of parameters, the regularization parameter $\alpha$ is initialized with 1 and reduced by a factor of 2 in each Newton step. The number of Newton steps and the damping factor $\beta$ were determined empirically with respect to the influence on accuracy and precision of the obtained $T_{1}$ values.

\subsubsection{T1 Quantitation}

After image reconstruction, $T_{1}$ quantitation was performed by fitting the following model pixel-wise to the magnitude images:

$$
M(t)=M_{s s}-\left(M_{0}+M_{s s}\right) \cdot \exp \left(-t / T_{1}^{*}\right)
$$


where $M_{s s}$ is the steady state magnetization, $M_{0}$ is the equilibrium magnetization and $T_{1}^{*}$ is the effective relaxation time. After fitting, with the assumption TR $\ll$ $T_{1}^{*}, T_{1}$, the desired $T_{1}$ was then calculated according to [64]:

$$
T_{1}=\frac{M_{0}}{M_{s s}} \cdot T_{1}^{*} .
$$

A pixelwise estimation of the parameters $M_{0}, M_{s s}$ and $T_{1}^{*}$ has been achieved by minimizing the cost function

$$
\phi(\boldsymbol{x})=\sum_{t}\left\|M_{t}(\boldsymbol{x})-y_{t}\right\|_{2}^{2}, \quad \text { with } \quad \boldsymbol{x}=\left(\begin{array}{c}
M_{s s} \\
M_{0} \\
T_{1}^{*}
\end{array}\right)
$$

where $t$ is the time index for each frame and $y_{t}$ is the corresponding signal intensity. Numerical computations employed a Levenberg-Marquardt algorithm in MATLAB R2013a (MathWorks, Natick, MA) and successful minimization was defined by reaching a maximum number of 3000 iterations, a relative change in the norm of the parameter update of less than $10^{-4}$, or a stepwise relative difference for the objective function value of less than $10^{-7}$. The ROI analyses were accomplished using the arrayShow tool [79] in MATLAB.

\subsubsection{Numerical Simulations}

To evaluate several acquisition and reconstruction parameters of the proposed method, a numerical phantom which composes of three circular objects in a circular background was built. The $k$-space data of this phantom was derived from the analytical Fourier space representation of an ellipse. Three different $T_{1}$ relaxation times ( $300 \mathrm{~ms}, 800 \mathrm{~ms}, 1500 \mathrm{~ms}$ ) with a background of $2000 \mathrm{~ms}$ were simulated. An array of 4 circular receiver coils was assumed, surrounding the phantom without overlap. The complex sensitivities of these coils were calculated based on the Biot-Savart's law and sinusoidal fitting [80]. Complex white Gaussian noise with a standard deviation of 0.1 was added to the simulated $k$-space data. The inversion recovery Look-Locker radial FLASH sequence was then used to sample the simulated $k$-space data with a base resolution of 192 pixels covering a FOV of $128 \mathrm{~mm}$, $\mathrm{TR} / \mathrm{TE}=2.93 / 2.01 \mathrm{~ms}, \mathrm{FA}=4^{\circ}$, total acquisition time $6 \mathrm{~s}$.

\subsubsection{MRI}

All MRI studies were performed at 3T (Magnetom Prisma, Siemens Healthcare, Erlangen, Germany). Young volunteers (age range 21 - 29 years) without known 
illness were recruited for studies during technical development as well as for preliminary testing and final acquisitions. Written informed consent, according to the recommendations of the local ethics committee, was obtained from all subjects prior to MRI. A commercial reference phantom as described in chapter 2 was employed for validation before subject studies. Phantom and brain studies employed the standard 64-channel head coil, while abdominal scans relied on an 18-element thorax coil in conjunction with 18 elements of the spine coil. Abdominal measurements were performed during a brief breath hold.

Fully sampled reference data sets for phantom and brain studies at $0.75 \times$ $0.75 \mathrm{~mm}^{2}$ spatial resolution were acquired by combing 21 sequential single-shot inversion recovery Look-Locker acquired data sets. The radial view-order for each individual inversion recovery acquisition was varied to yield a uniform distribution of 441 spokes per time point after combination. Image reconstruction was then performed by density compensation, gridding, and inverse FFT of the respective raw data. Individual coil images were combined by a matched filter based technique [40] prior to pixel-wise fitting.

\subsection{Results}

\subsubsection{Numerical Simulations}

\section{Number of Spokes}

For image-space based $T_{1}$ mapping, the choice of number of spokes per frame not only determines the quality of the reconstructed image but also influences the temporal resolution of inversion recovery. The latter is crucial for determination of short $T_{1}$ values. A proper choice then helps to ensure the reconstructed image to be artifact-free while keeping the temporal resolution. To demonstrate this effect, Figure 5.2 (a) shows $T_{1}$ maps estimated from undersampled data ranging from 5 spokes (undersampling factor 60) to 29 spokes per frame (undersampling factor 10) with a step size of 8 . Streaking artifacts are observed when the binning size is reduced to be 13 and become more severe in the extreme case of 5 spokes. $T_{1}$ maps from 21 and 29 spokes are free of streaking artifacts. Figure 5.2 (b) plots the quantitative values of $T_{1}$ maps for the binning size ranging from 13 to 117. Estimated $T_{1}$ values are consistent with the ground truth when the number of spokes is chosen from 13 to 61 . A tendency of overestimation is observed for larger binning sizes, indicating that lower temporal resolution can cause bias in $T_{1}$ estimation. In order to balance image quality and temporal resolution, 13 to 45 spokes per frame are recommended for the purpose of $T_{1}$ mapping. 
(a)

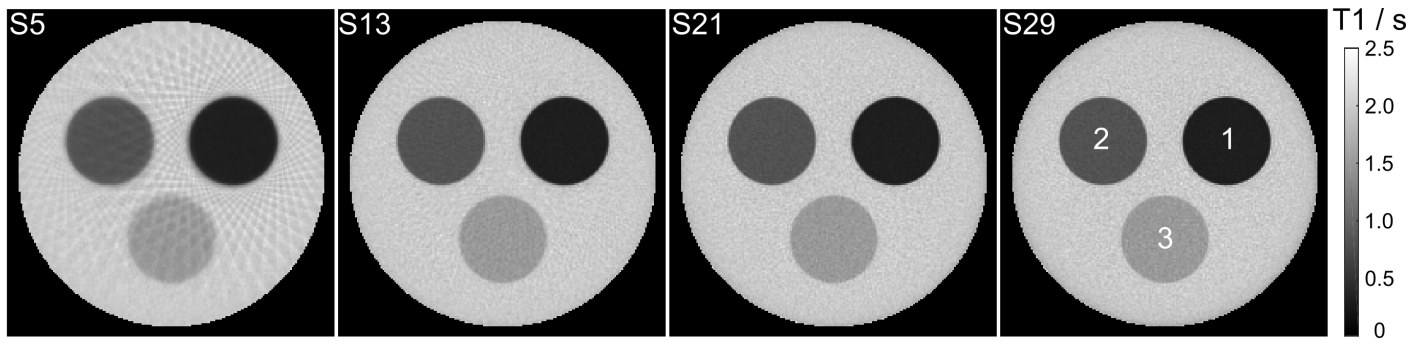

(b)

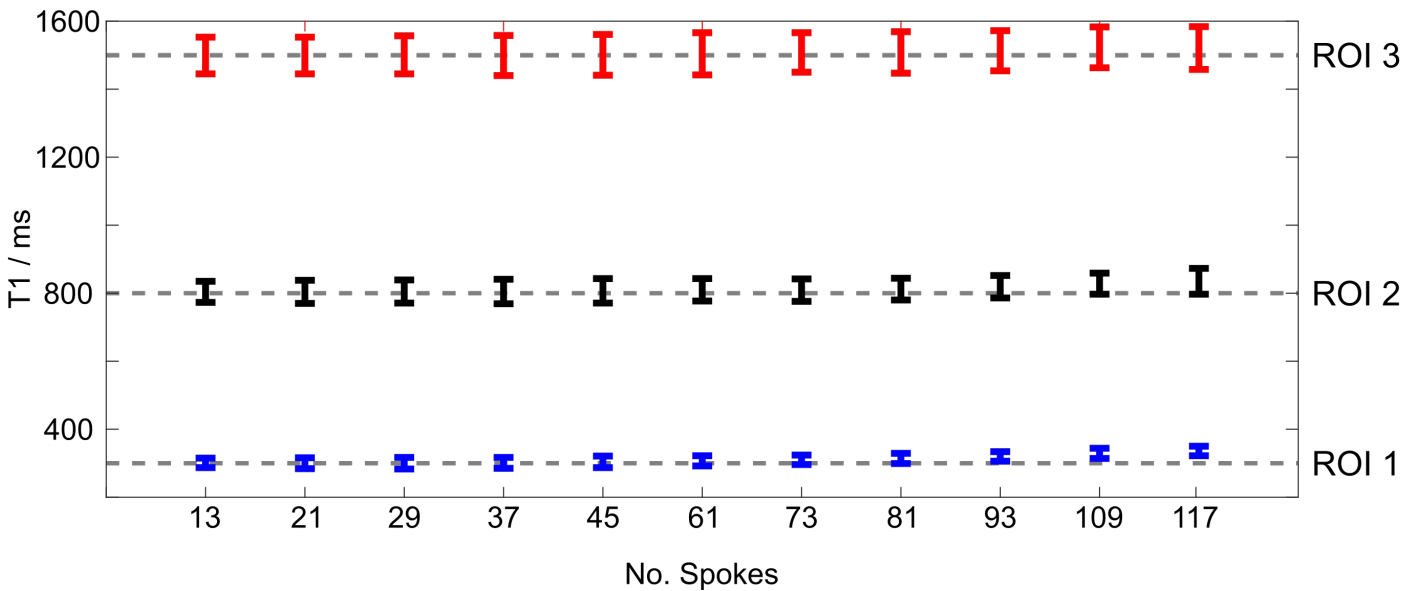

Figure 5.2: (a) $T_{1}$ maps with four different binning sizes. (b) Quantitative ROI analyses of $T_{1}$ maps using binning sizes from 13 to 117 spokes with the proposed method. Dashed lines and error bar represent ground truth and standard deviation respectively.

\section{Reconstruction Parameters}

The two main reconstruction parameters (number of iterations and temporal damping factor) of the proposed method were investigated using the simulated phantom. The number of spokes was set to be 21 per frame. Figure 5.3 shows quantitative $T_{1}$ values at different iterations and variable damping factors. With a lower damping factor, more iterations are needed for the reconstruction to converge to the true solution, i.e., to have a good $T_{1}$ accuracy. A high damping factor accelerates the convergence but at a cost of increasing the standard deviation. In order to accelerate the convergence while keeping a good precision, a damping factor of 0.9 was then chosen for all studies. After fixing the damping factor to be $0.9,6$ or 7 Newton steps should be employed.

Finally, Figure 5.4 shows validated results on the numerical phantom using 21 spokes per frame and reconstructed with a damping factor 0.9 and 7 Newton steps. Figure 5.4 (Left) are four images at different inversion times reconstructed by the proposed method. Fitting curves of three representative pixels are plotted in the middle. The three estimated parameter maps together with the corresponding $T_{1}$ map are presented on the right after pixel-wise fitting. The quantitative results of the corresponding $T_{1}$ map are shown in Table 5.1, which confirm the accuracy of 

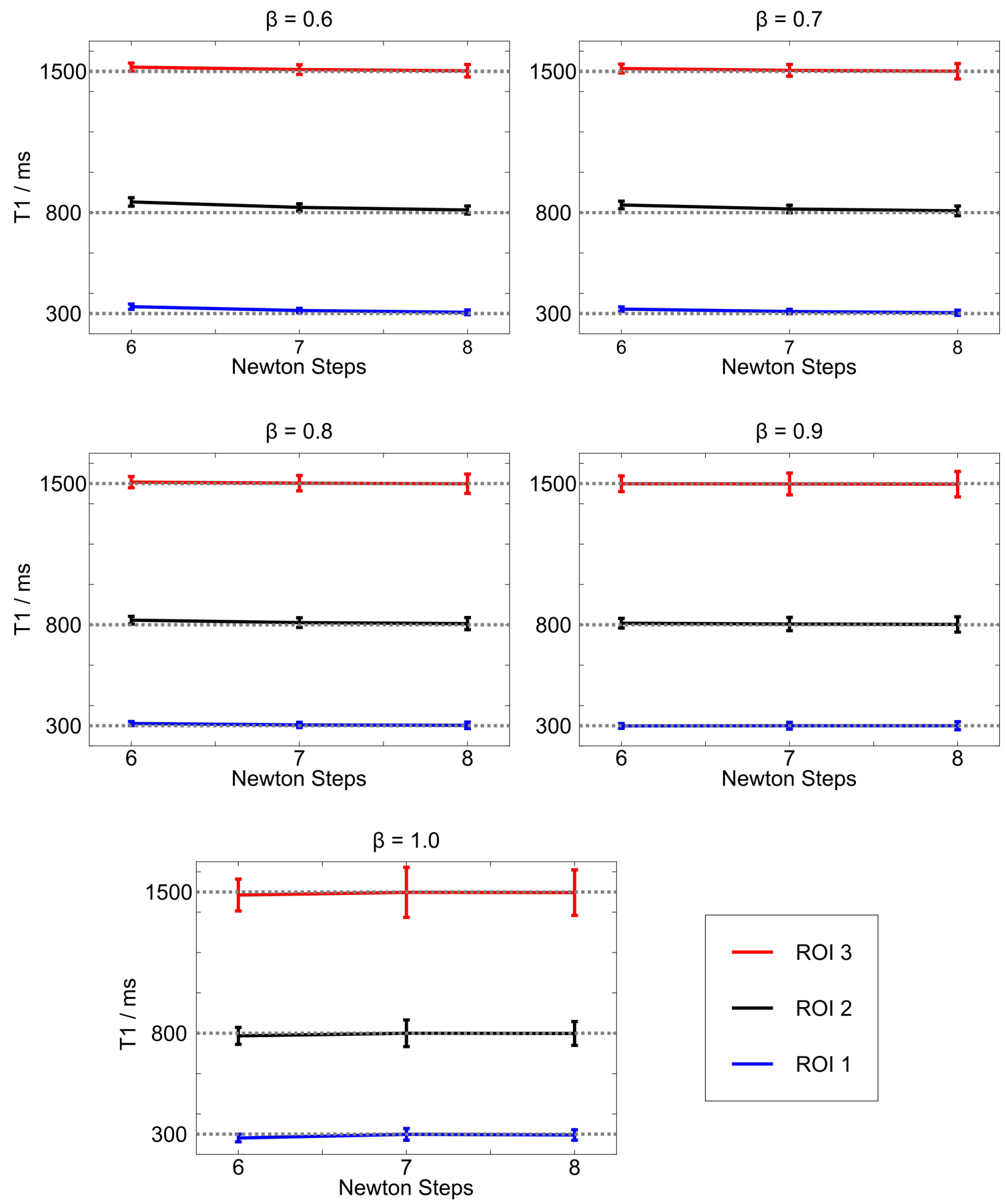

Figure 5.3: Effects of damping factors and Newton steps of the proposed method on the quantitative results for a numerical phantom. Dashed lines and error bar represent ground truth and standard deviation, respectively. Connecting solid lines are drawn to guide the eye. 

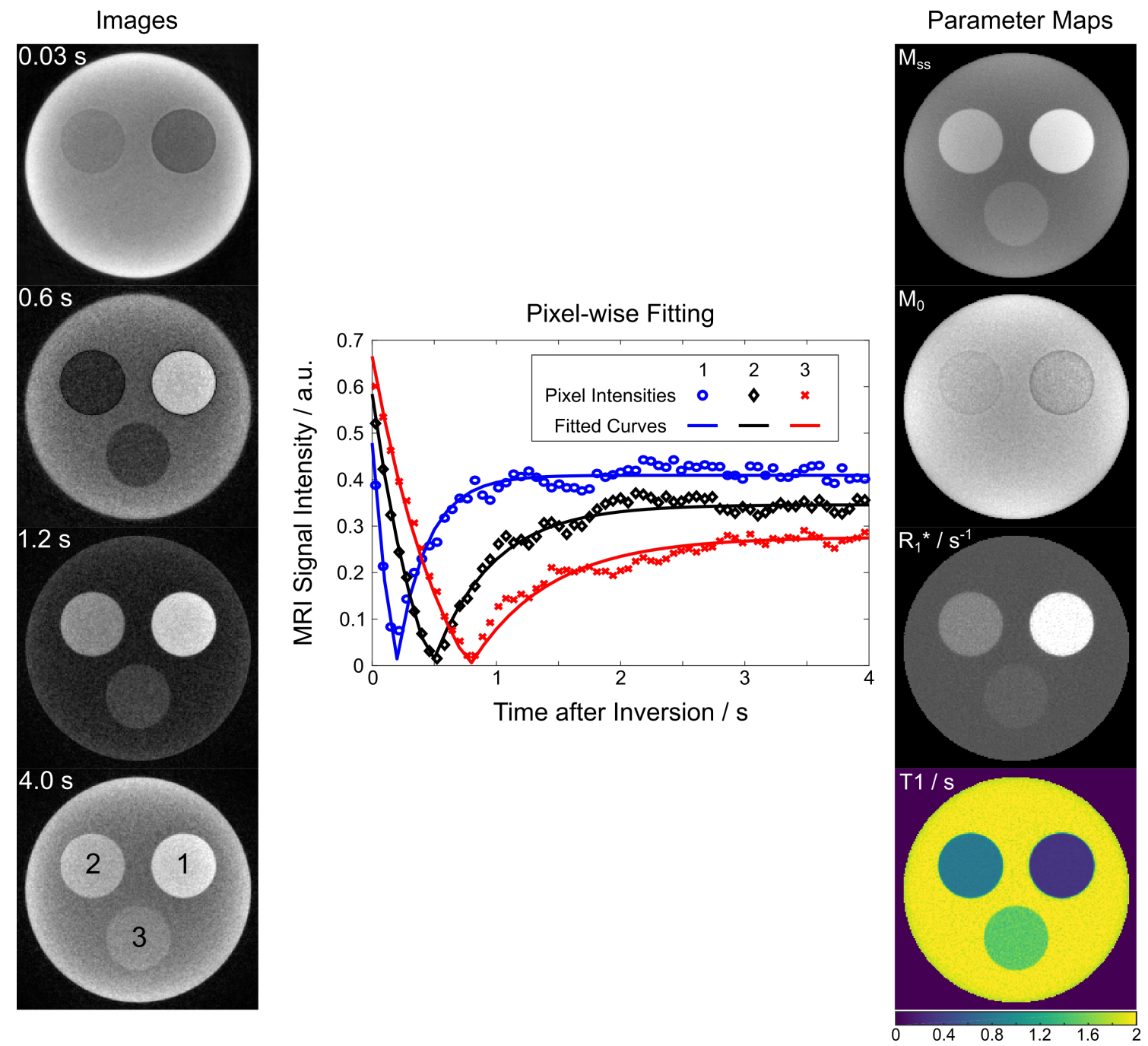

Figure 5.4: Validation of the proposed method in a numerical phantom study. (Left) Four representative images during inversion recovery reconstructed by the proposed method. (Middle) Signal intensity time courses and respective fitting results of the simulated phantom with $T_{1}=300 \mathrm{~ms}$ (blue), $800 \mathrm{~ms}$ (red) and $1500 \mathrm{~ms}$ (black). (Right) The pixel-wise fitted three parameter maps and the corresponding $T_{1}$ map. The quantitative ROI evaluations of the $T_{1}$ map are shown in Table 5.1. Images during inversion recovery were reconstructed using 7 Newton steps with a damping factor of 0.9 . 
Table 5.1: Quantitative ROI analysis of simulated $T_{1}$ maps shown in Figure 5.4.

\begin{tabular}{ccr}
\hline ROI & True $T_{1}$ Value & \multicolumn{1}{c}{ NLINV $^{1}$} \\
\hline 1 & 300 & $300 \pm 16$ \\
2 & 800 & $804 \pm 34$ \\
3 & 1500 & $1499 \pm 54$ \\
Background & 2000 & $1992 \pm 69$ \\
\hline${ }^{1}$ Results represent mean & values \pm \\
\multicolumn{2}{c}{ standard deviations. }
\end{tabular}

the proposed method when compared to the ground truth.

\subsection{2 in vitro Studies}

\section{Reconstruction Parameters}

The number of iterations was investigated on the experimental phantom after fixing the damping factor to be 0.9 . Figure 5.5 shows phantom $T_{1}$ maps and the corresponding quantitative $T_{1}$ values along Newton steps. High number of iterations induces more noise in the maps which is reflected as higher standard deviation in the quantitative results. 6 or 7 Newton steps provides a good balance between accuracy and precision. Generally in this study, 6 Newton steps has been used for the study of in-plane resolution lower or equal than $1 \times 1 \mathrm{~mm}^{2}$. For applications with submillimeter in-plane resolution, 7 Newton steps was employed to ensure the convergence.

\section{Comparison to Fully-sampled Data}

$T_{1}$ map by the proposed method was then compared to the corresponding map from the fully sampled data set as shown in Figure 5.6. Visual inspection reveals that $T_{1}$ map by the proposed method is in a good agreement with the reference but exhibits a slightly increased noise level. Despite the higher standard deviation, the quantitative results in Table 5.2 confirm the accuracy of the proposed method when compared to the fully sampled measurement.

\subsection{3 in vivo Studies}

\section{Number of Spokes}

The binning size was also validated on brain studies. Figure 5.7 shows brain $T_{1}$ maps using different number of spokes ranging from 11 to 21 , which are com- 
(a)
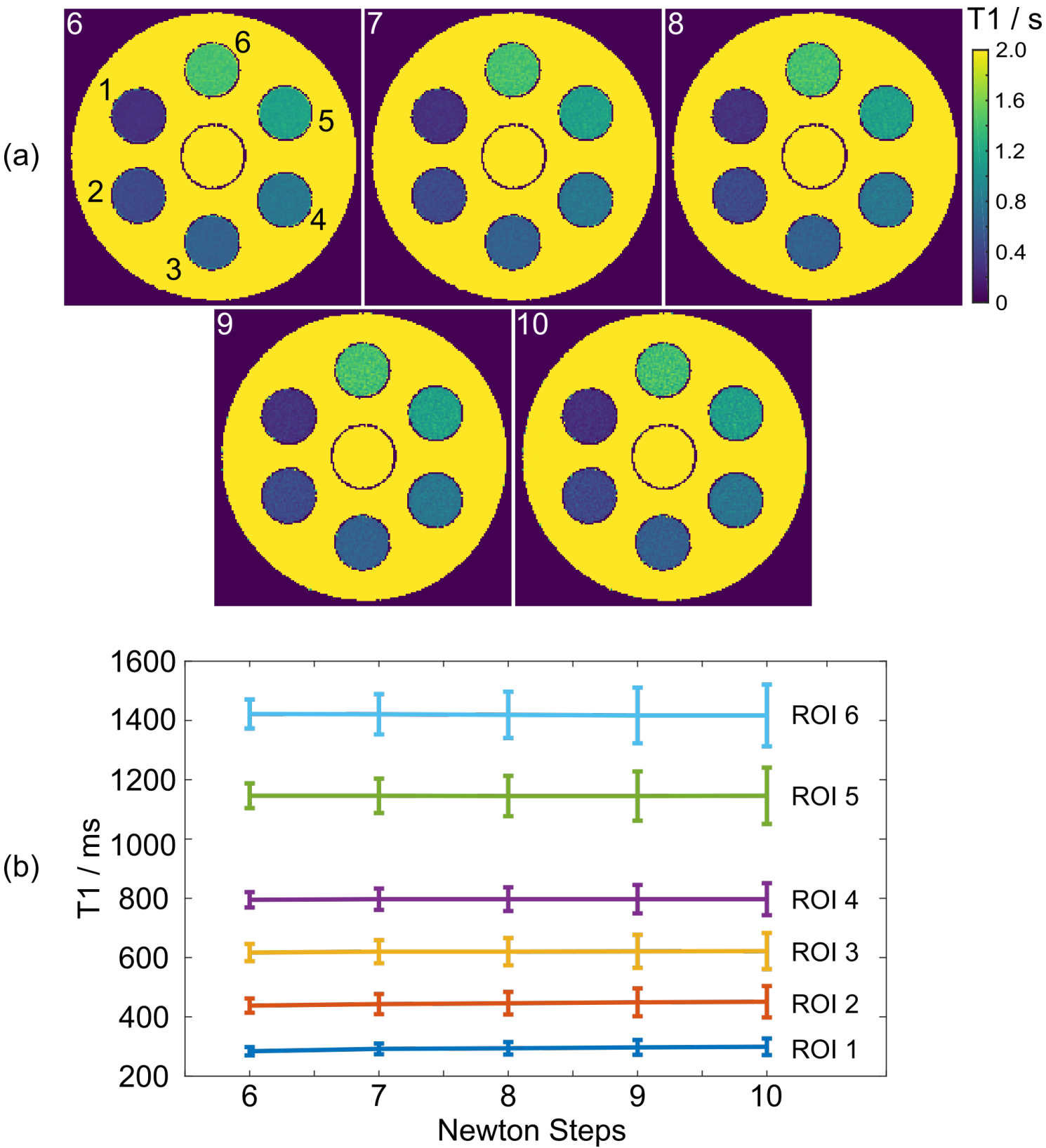

Figure 5.5: Phantom $T_{1}$ maps (a) and the corresponding quantitative ROI evaluations (b) along with Newton steps (6 to 10). Connecting solid lines in (b) are drawn to guide the eye. Measurement parameters: spatial resolution $0.75 \times 0.75 \times 5 \mathrm{~mm}^{3}$, TR/TE $/ \alpha=2.93 / 2.01 \mathrm{~ms} / 4^{\circ}, 21$ spokes/frame. 


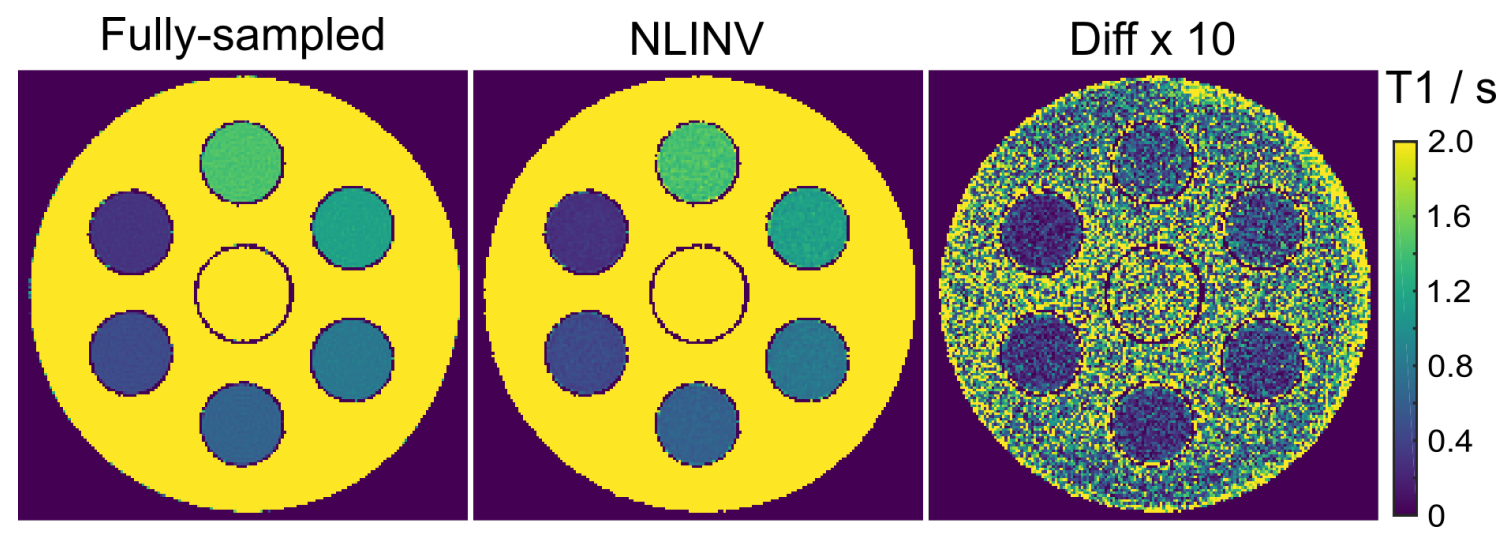

Figure 5.6: Comparison of phantom $T_{1}$ map estimated from a fully sampled data set and that from a single-shot data set by the proposed method. The corresponding $T_{1}$ difference map $(\times 10)$ is shown in the right. Measurement parameters: spatial resolution $0.75 \times 0.75 \times 5 \mathrm{~mm}^{3}$, $\mathrm{TR} / \mathrm{TE} / \alpha=2.93 / 2.01 \mathrm{~ms} / 4^{\circ}, 21$ spokes/frame.

Table 5.2: Quantitative $T_{1}$ values (ms) ${ }^{1}$ of the experimental phantom and brain $T_{1}$ maps $^{2}$.

\begin{tabular}{rcrr}
\hline & Tube & Fully-sampled & \multicolumn{1}{c}{ NLINV } \\
\hline \multirow{4}{*}{ Phantom } & 1 & $308 \pm 17$ & $299 \pm 25$ \\
& 2 & $462 \pm 20$ & $456 \pm 36$ \\
& 3 & $638 \pm 25$ & $628 \pm 33$ \\
& 4 & $810 \pm 27$ & $800 \pm 44$ \\
& 5 & $1160 \pm 31$ & $1152 \pm 57$ \\
Brain & 6 & $1445 \pm 35$ & $1428 \pm 62$ \\
\hline \multirow{6}{*}{ WM } & $751 \pm 42$ & $752 \pm 82$ \\
& GM & $1308 \pm 58$ & $1288 \pm 92$ \\
\hline
\end{tabular}

${ }^{1}$ Results represent mean values \pm standard deviations.

${ }^{2}$ Regions-of-interest are indicated in Figure 5.5 (phantom) and Figure 5.8 (brain). 
Table 5.3: Quantitative ROI analysis of the brain $T_{1}$ maps (ms) shown in Figure

\begin{tabular}{|c|c|c|c|c|c|c|}
\hline & 11 & 13 & 15 & 17 & 19 & 21 \\
\hline WM & $707 \pm 60$ & $711 \pm 71$ & $709 \pm 66$ & $710 \pm 70$ & $711 \pm 68$ & $711 \pm 72$ \\
\hline GM & $1362 \pm 98$ & $1373 \pm 94$ & $1376 \pm 116$ & $1358 \pm 92$ & $1360 \pm 99$ & $1349 \pm 92$ \\
\hline
\end{tabular}

monly employed in real-time MRI applications. There is no visible difference among the $T_{1}$ maps. The quantitative ROI analyzed results of white matter (WM) and gray matter $(\overline{\mathrm{GM}})$ of all the six maps are presented in Table 5.3 , which confirm the consistency among $T_{1}$ maps acquired from the chosen number of spokes. Both the qualitative and quantitative results reveal that the proposed method is tolerant to high undersampling factors (11 spokes corresponds to an undersampling factor of 36) and therefore allows a relative freedom of choice of spokes. Noteworthy, a higher undersampling factor has been achieved in the brain studies than in the simulation studies. This is mainly because there are more physical coils placed around the object which allows a further acceleration.
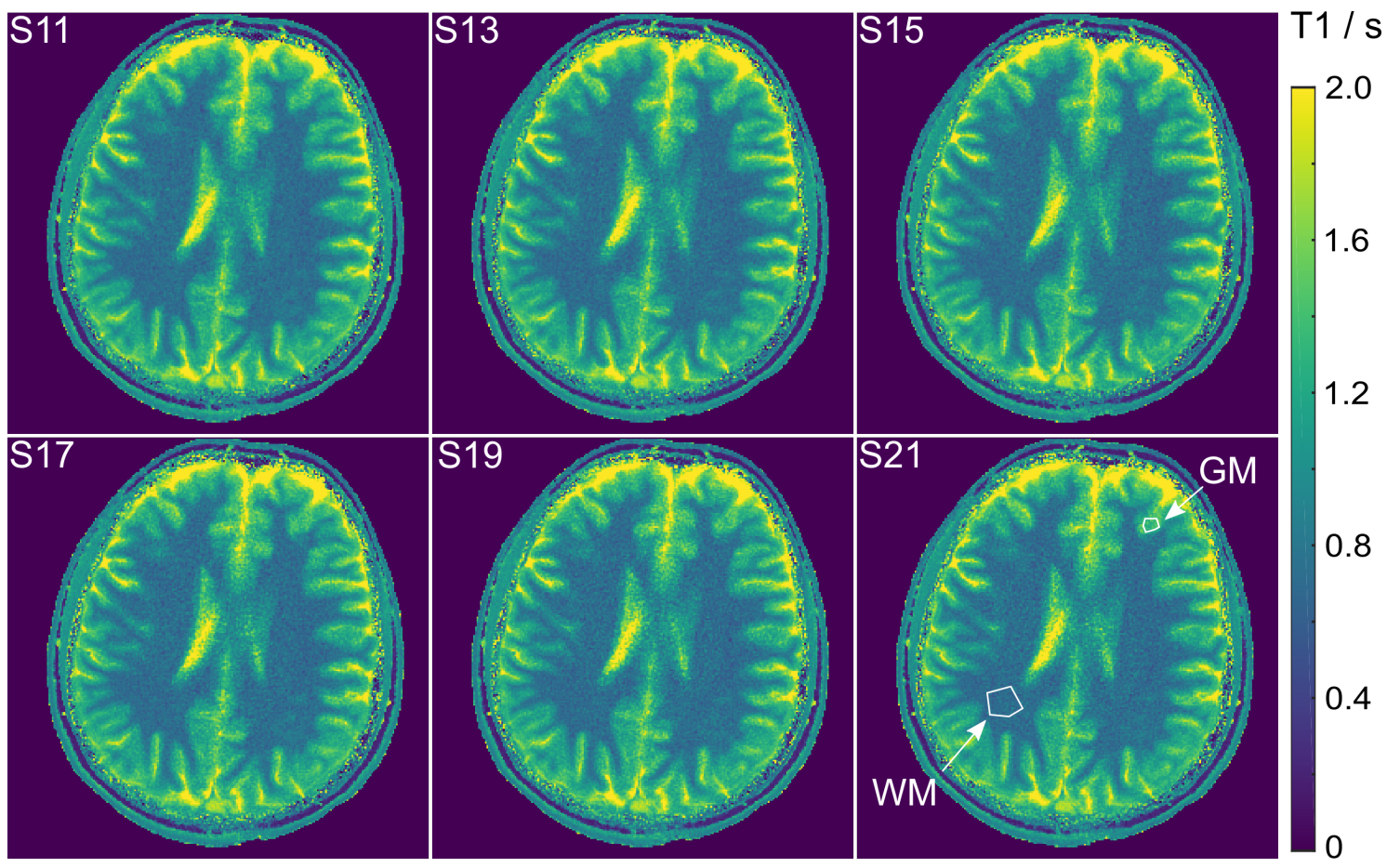

Figure 5.7: Brain $T_{1}$ maps acquired using different number of spokes (11 to 21). White arrows indicate selected regions-of-interest for quantitative analyses of white matter (WM) and gray matter (GM) respectively. Measurement parameters: spatial resolution $0.75 \times 0.75 \times 5 \mathrm{~mm}^{3}$, $\mathrm{TR} / \mathrm{TE} / \alpha=2.57 / 1.68 \mathrm{~ms} / 4^{\circ}$. 


\section{Reconstruction Parameters}

Figure 5.8 shows brain $T_{1}$ maps and the corresponding quantitative evaluations at different Newton steps. Similar to experimental phantom results, high number of iterations introduces more noise in the maps which is then reflected as a higher standard deviation in the quantitative results. 7 Newton steps provides a good balance between $T_{1}$ accuracy and precision for this submillimeter application.

(a)

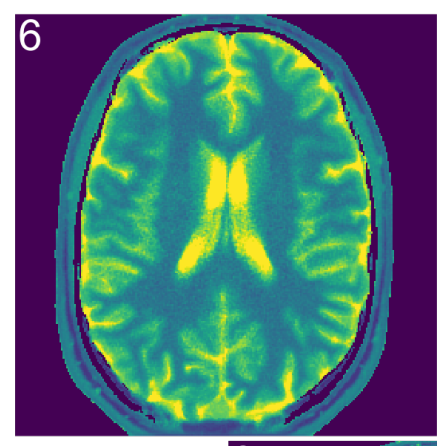

9
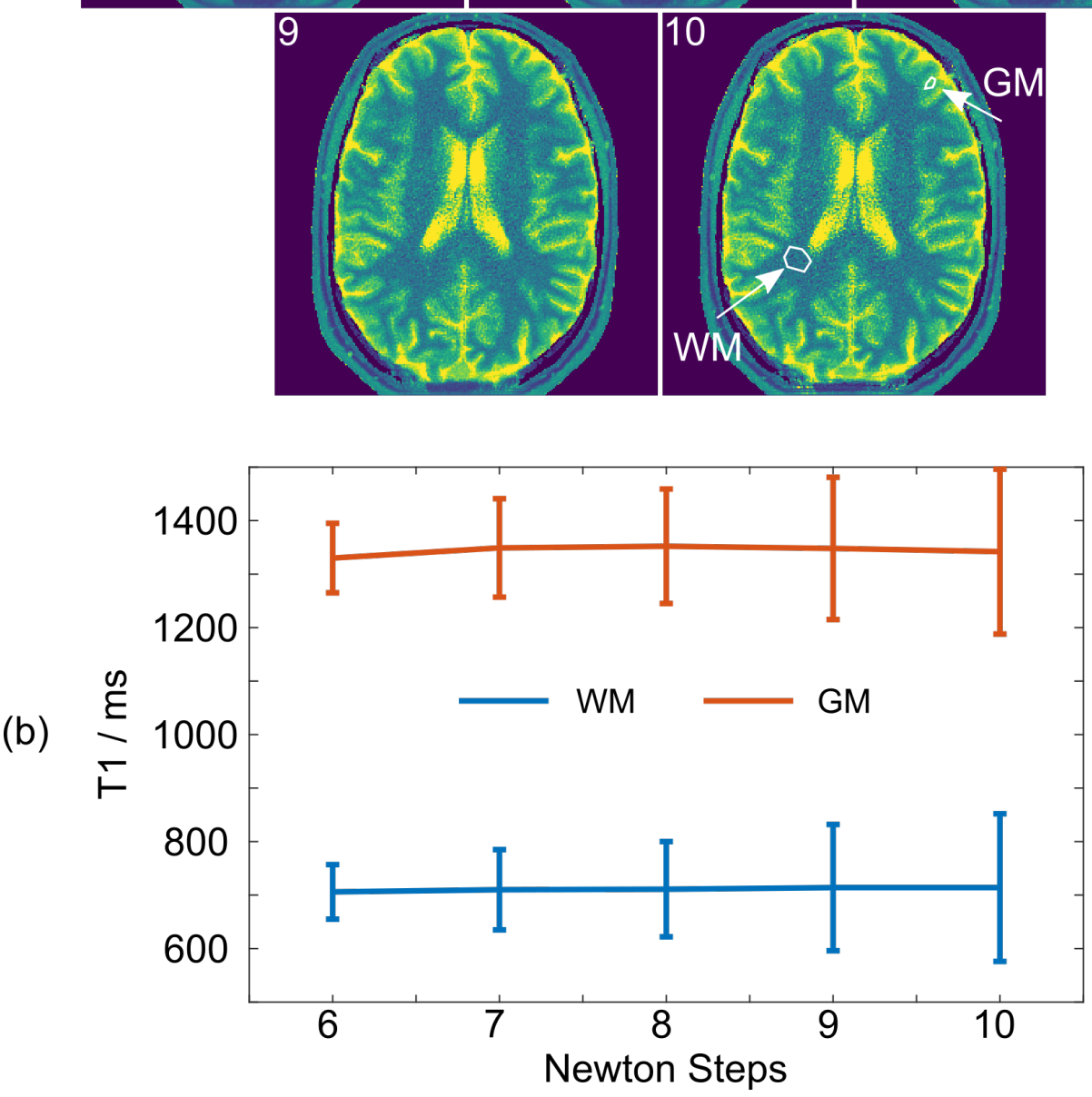

Figure 5.8: Human brain $T_{1}$ maps (a) and the corresponding quantitative results (b) along with Newton steps (6 to 10). White arrows in (a) indicate selected regions-of-interest for quantitative analyses of white matter (WM) and gray matter (GM) respectively. Connecting lines in (b) are drawn to guide the eye. Measurement parameters: spatial resolution $0.75 \times 0.75 \times 5 \mathrm{~mm}^{3}, \mathrm{TR} / \mathrm{TE} / \alpha=2.93 / 2.01 \mathrm{~ms} / 4^{\circ}, 21$ spokes/frame. 


\section{Comparison to Fully-sampled Data}

A comparison of brain $T_{1}$ map estimated from the fully sampled data set and that from a single-shot data set by the proposed method is presented in Figure 5.9. In line with the phantom results, the $T_{1}$ map by the proposed method is in a good agreement with that from the fully sampled data set but exhibits a slight higher noise level. Again, despite the higher standard deviation, the quantitative results of white matter and gray matter shown in Table 5.2 confirm the general accuracy of the proposed method on brain studies.
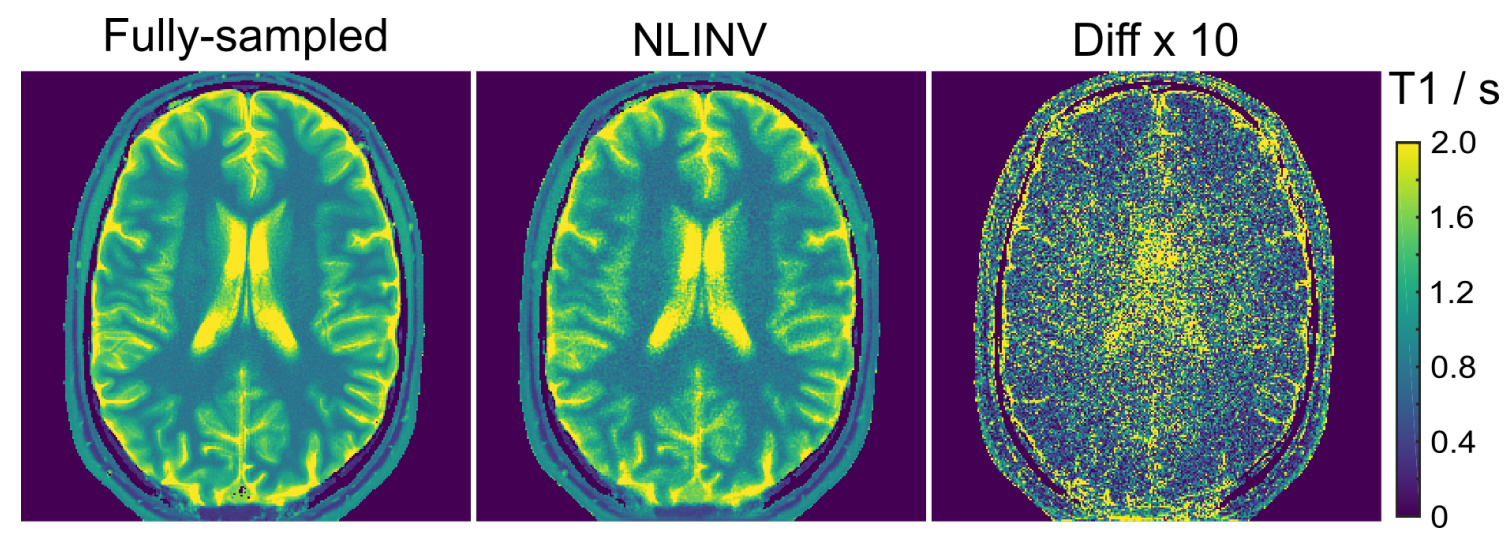

Figure 5.9: Comparison of a brain $T_{1}$ map estimated from a fully sampled data set and that from a single-shot data set by the proposed method. The corresponding $T_{1}$ difference map $(\times 10)$ is shown in the right. Measurement parameters: spatial resolution $0.75 \times 0.75 \times 5 \mathrm{~mm}^{3}$, $\mathrm{TR} / \mathrm{TE} / \alpha=2.93 / 2.01 \mathrm{~ms} / 4^{\circ}, 21$ spokes/frame.

\subsection{Extensions}

\subsubsection{Modified Non-local Means (NLM) Filter}

To improve the precision of $T_{1}$ maps, a recently proposed modified non-local means (NLM) filter [54] was further employed to denoise the reconstructed images prior to pixel-wise fitting. The filter preserves small isolated details and efficiently removes background noise (corresponding to a $60 \%$ SNR improvement) without introducing blur, smearing or patch artifacts. This is accomplished by extending the conventional non-local means algorithm to adapt the influence of the original pixel value according to a simple measure for patch regularity. Detail preservation is improved by a compactly supported weighting kernel which closely approximates the commonly used exponential weight [54]. An example of the denoising effect on reconstructed images and $T_{1}$ map is shown in Figure 5.10. A clear SNR improvement is observed in both images and $T_{1}$ maps. Moreover, when 


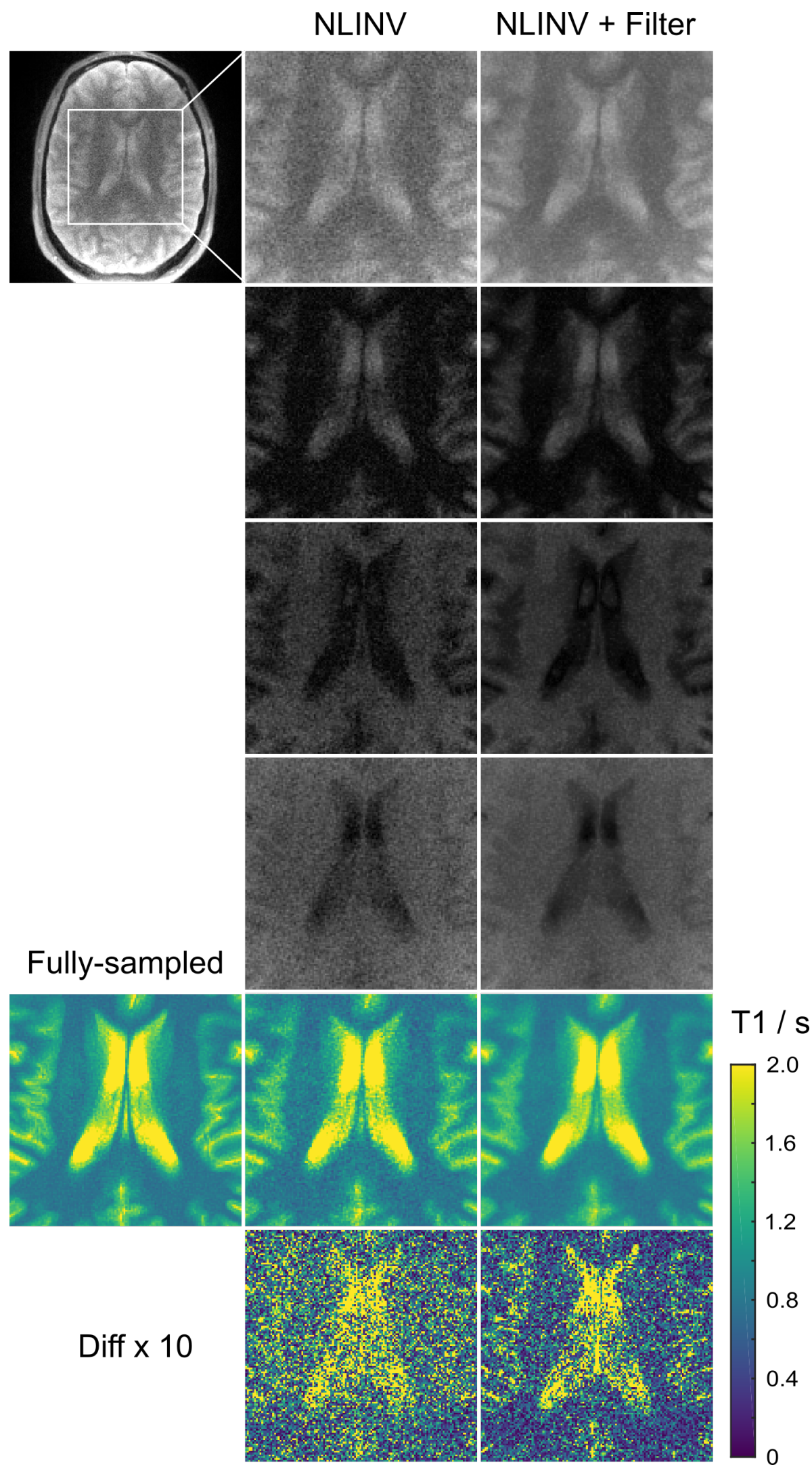

Figure 5.10: Reconstructed images with different contrasts (enlarged, rows 1-4) during inversion recovery together with $T_{1}$ maps (row 5) before and after the application of the denoising filter. The enlarged $T_{1}$ difference maps $(\times 10)$ are shown in the last row. Measurement parameters: spatial resolution $0.75 \times 0.75 \times 5 \mathrm{~mm}^{3}$, TR/TE $/ \alpha=$ $2.93 / 2.01 \mathrm{~ms} / 4^{\circ}, 21$ spokes/frame. 
Table 5.4: Acquisition parameters for single-slice and multi-slice $T_{1}$ mapping.

\begin{tabular}{|c|c|c|c|c|}
\hline & \multicolumn{2}{|c|}{ Brain/Phantom } & \multicolumn{2}{|c|}{ Abdomen } \\
\hline & 1-slice & Multi-slice & 1-slice & Multi-slice \\
\hline Field-of-view / mm² & $192 \times 192$ & $192 \times 192$ & $320 \times 320$ & $320 \times 320$ \\
\hline Image matrix size & $384 \times 384$ & $256 \times 256$ & $320 \times 320$ & $212 \times 212$ \\
\hline Resolution $/ \mathrm{mm}^{2}$ & $0.5 \times 0.5$ & $0.75 \times 0.75$ & $1.0 \times 1.0$ & $1.5 \times 1.5$ \\
\hline Slice thickness / mm & 5 & 5 & 8 & 8 \\
\hline Repetition time (TR) / ms & 3.37 & 2.64 & 2.25 & 1.93 \\
\hline Echo time (TE) / ms & 2.25 & 1.76 & 1.47 & 1.25 \\
\hline Bandwidth / Hz pixel ${ }^{-1}$ & 720 & 1090 & 1420 & 1970 \\
\hline Flip angle / degree & 4 & 4 & 4 & 4 \\
\hline Spokes per frame & 19 & 15 & 15 & 11 \\
\hline Time per frame / ms & 64.0 & 39.6 & 33.8 & 21.2 \\
\hline
\end{tabular}

compared to the $T_{1}$ map from the fully sampled case, the $T_{1}$ map after denoising results in less residual errors.

\subsubsection{Single-shot Multi-slice T1 mapping}

Another extension of the above method is the simultaneous multi-slice $T_{1}$ mapping within one single-shot. There are two main spoke distribution schemes for multislice data acquisition after a single non-selective inversion pulse, i.e., sequential and interleaved modes as depicted in Figure 5.11. The sequential multi-slice acquisition scheme is favored because it is advantageous to keep the temporal footprint of a single frame as small as possible to maintain temporal fidelity. Unlike the interleaved mode where the signal relaxation follows the same model as for the single-slice case (equation (5.3) ) with a larger time spacing, i.e., the product of the number of slices and the repetition time, a particular slice in the sequential based data acquisition mode experiences periods of free relaxation governed by pure $T_{1}$ relaxation and interleaved with periods governed by $T_{1}^{*}$. Therefore, the signal relaxation is not compatible with the previous model in equation (5.3). An analytical expression for this process was then derived in [81] based on a similar structure of multi-slice spin locking [82]. As only $\left(M_{0}, M_{s s}, R_{1}^{*}\right)$ are unknowns in the relaxation formula [81], $T_{1}$ can be calculated according to equation (5.4) after a three parameter pixel-wise fitting. The accuracy of multi-slice $T_{1}$ mapping was then validated by comparison of the estimated $T_{1}$ maps to those obtained from single-slice acquisitions for experimental phantom, human brain and abdomen studies. Further, a long-TR IR fast spin-echo (FSE) sequence with 13 logarithmically spaced inversion times between 50 and $2300 \mathrm{~ms}$ served as a gold standard for 

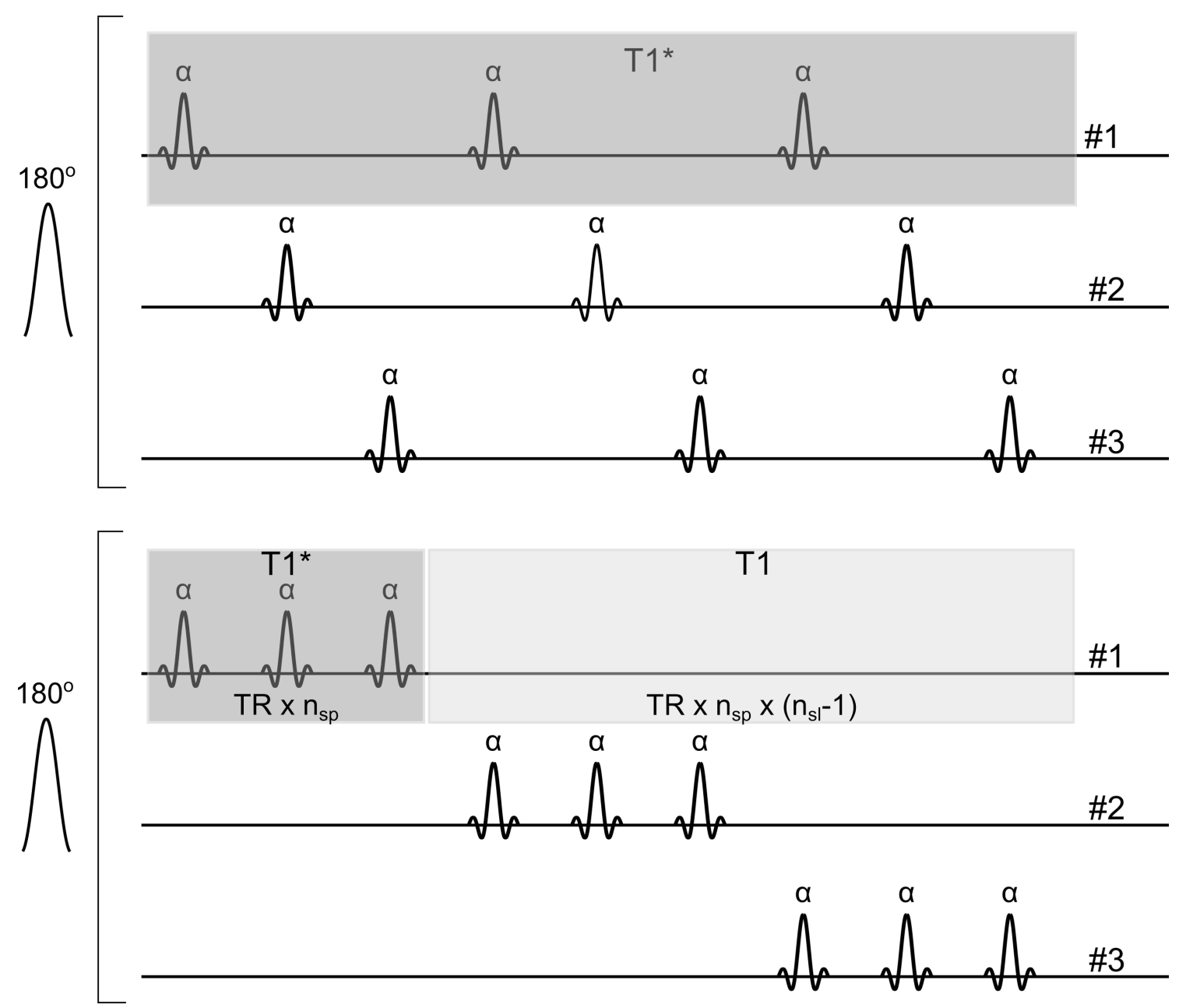

Figure 5.11: Acquisition schemes for spoke-interleaved (top) and spoke-sequential (bottom) three-slice measurements after a non-selective adiabatic inversion pulse. 
Table 5.5: $T_{1}$ relaxation times ${ }^{1}$ of a reference phantom obtained by single-slice and multi-slice $T_{1}$ mapping.

\begin{tabular}{ccccc}
\hline ROI $^{2}$ & 1 Slice & 3 Slices & 5 Slices & IR-FSE $^{3}$ \\
\hline 1 & $0.32 \pm 0.03$ & $0.31 \pm 0.03$ & $0.30 \pm 0.03$ & $0.33 \pm 0.01$ \\
2 & $0.48 \pm 0.04$ & $0.46 \pm 0.04$ & $0.46 \pm 0.04$ & $0.50 \pm 0.01$ \\
3 & $0.66 \pm 0.06$ & $0.65 \pm 0.05$ & $0.64 \pm 0.06$ & $0.68 \pm 0.01$ \\
4 & $0.83 \pm 0.07$ & $0.82 \pm 0.06$ & $0.80 \pm 0.07$ & $0.86 \pm 0.01$ \\
5 & $1.22 \pm 0.07$ & $1.22 \pm 0.06$ & $1.20 \pm 0.07$ & $1.25 \pm 0.01$ \\
6 & $1.49 \pm 0.09$ & $1.48 \pm 0.08$ & $1.47 \pm 0.10$ & $1.50 \pm 0.01$ \\
\hline
\end{tabular}

${ }^{1}$ All values (mean \pm SD) are given in s. Multi-slice data refer to the same section.

${ }^{2}$ Region-of-interest as indicated in Figure 5.12 .

${ }^{3}$ Reference data obtained by a long-TR (7.2 s) inversion recovery fast spin-echo MRI sequence.

$T_{1}$ determination $(\mathrm{TR}=7.2 \mathrm{~s}, \mathrm{TE}=12 \mathrm{~ms}, 6$ echoes, measuring time $=50 \mathrm{~min}$ ) of the experimental phantom study. The acquisition parameters for single-slice and multi-slice acquisitions are summarized in Table 5.4 .

Figure 5.12 shows proton density and $T_{1}$ maps for a $T_{1}$ reference phantom. The results were obtained for a single-slice acquisition at $0.5 \mathrm{~mm}$ in-plane resolution as well as for a 3-slice and 5-slice acquisition at $0.75 \mathrm{~mm}$ in-plane resolution. In all cases the maps correspond to the same section. Visual inspection reveals almost identical image quality and color-coded $T_{1}$ values apart from a slightly enhanced noise level for 5 -slice acquisitions. Corresponding single-pixel intensity time courses and fitting results are shown in Figure 5.13 for three selected tubes with $T_{1}$ values of $0.33 \mathrm{~s}, 0.68 \mathrm{~s}$ and $1.25 \mathrm{~s}$ (tubes 1,3 and 5 in Figure 5.12). The numerical results summarized in Table 5.5 reveal accurate $T_{1}$ determinations for both small and large $T_{1}$ values when compared to a reference measurement using an IR fast spin-echo (FSE) MRI sequence at long TR (7.2 s). Multi-slice acquisitions show a mild tendency towards lower $T_{1}$ relaxation times with increasing number of sections, while all values remain within one standard deviation.

In close analogy to the phantom data, Figure 5.14 shows proton density and $T_{1}$ maps for a single-slice ( $0.5 \mathrm{~mm}$ in-plane resolution), 3-slice and 5 -slice acquisition ( $0.75 \mathrm{~mm}$, same section selected) of the human brain. Apart from minor increased noise level for a larger number of slices, all maps are of similar quality. This finding is confirmed by the numerical results in Table 5.6 for brain (frontal and occipital white and gray matter), liver, and kidney (cortex and medulla) studies. The present results are then compared to some most recent literature findings [83-85]. As it turned out, all $T_{1}$ values are close to published data. Finally, Figure 5.15 shows all simultaneously acquired $T_{1}$ maps of a transverse 5 -slice acquisition 


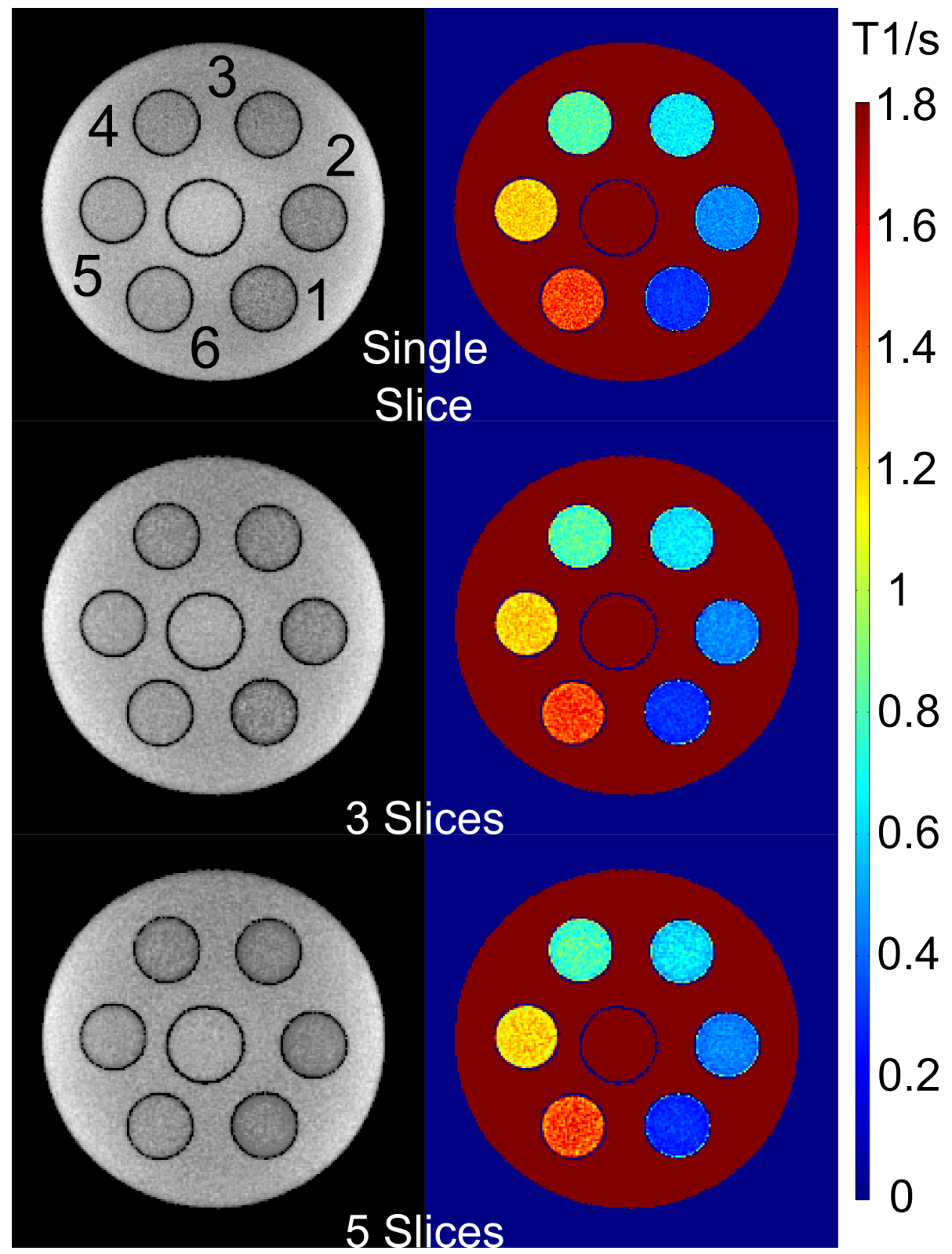

Figure 5.12: Single-shot proton density and $T_{1}$ maps obtained for a $T_{1}$ reference phantom. (Top) Single-slice acquisition at $0.5 \mathrm{~mm}$ in-plane resolution, (middle) 3-slice and (bottom) 5-slice acquisition at $0.75 \mathrm{~mm}$ in-plane resolution (same section). For experimental details see Table 5.4. numerical results for tubes 1 to 6 are given in Table 5.5 . 
Table 5.6: $T_{1}$ relaxation times of the human brain, liver and kidney obtained by single-slice and multi-slice $T_{1}$ mapping.

\begin{tabular}{lcccc}
\hline Tissue & 1 Slice & 3 Slices & 5 Slices & Literature \\
\hline Frontal WM & $0.73 \pm 0.11$ & $0.73 \pm 0.10$ & $0.74 \pm 0.12$ & $0.80-0.88[83]$ \\
Occipital WM & $0.78 \pm 0.08$ & $0.78 \pm 0.09$ & $0.78 \pm 0.08$ & \\
Frontal GM & $1.36 \pm 0.14$ & $1.39 \pm 0.10$ & $1.38 \pm 0.13$ & $1.35-1.50[$ [83] \\
Occipital GM & $1.49 \pm 0.12$ & $1.48 \pm 0.11$ & $1.48 \pm 0.07$ & \\
Liver & $0.68 \pm 0.05$ & $0.68 \pm 0.05$ & $0.67 \pm 0.04$ & $0.76 \pm 0.15[$ [84] \\
Kidney cortex & $1.30 \pm 0.08$ & $1.26 \pm 0.07$ & $1.27 \pm 0.08$ & $1.38 \pm 0.10[[85]$ \\
Kidney medulla & $1.75 \pm 0.09$ & $1.75 \pm 0.08$ & $1.77 \pm 0.05$ & $1.65 \pm 0.09$ [85] \\
\hline
\end{tabular}

All values (mean \pm SD) are given in s. Multi-slice data refer to the same section.

of the liver at $1.5 \mathrm{~mm}$ in-plane resolution.

\subsection{Discussion}

A reliable and robust single-shot $T_{1}$ mapping technique based on radial undersampling and iterative image reconstruction has been developed. Different acquisition parameters and the choice of reconstruction parameters have been validated on simulation, experimental phantom, human brain and abdomen studies. The proposed method can achieve a pronounced high undersampling factor and offers a relative freedom of user-selective choices of spatial and temporal resolution. The benefit of a high temporal resolution also enables an extension for simultaneous multi-slice $T_{1}$ mapping up to 5 sections. Further, a modified non-local means (NLM) filter recently developed for real-time MRI can be used for the improvement of $T_{1}$ precision. Accuracy of the proposed method has been confirmed by comparisons to ground truth for simulation studies, to $T_{1}$ maps from fully-sampled and the IR FSE sampled data sets for phantom studies and to $T_{1}$ maps from fullysampled data set for brain studies respectively. Preliminary in vivo applications to human brain, liver and kidney are in agreement with literature values.

Although a number of approaches have been described using undersampled radial acquisition schemes in conjunction with sliding-window and parallel imaging reconstructions [73, 74, 86] or model-based approaches [76, 87], so far they only allow for single-slice applications. Moreover, respective studies of a phantom or human brain resulted in much lower spatial resolution of $1 \times 1 \times 8 \mathrm{~mm}^{3}=8 \mathrm{~mm}^{3}$ [73], $1.8 \times 1.8 \times 5 \mathrm{~mm}^{3}=16.2 \mathrm{~mm}^{3}$ [86], $1.56 \times 1.56 \times 10 \mathrm{~mm}^{3}=24.3 \mathrm{~mm}^{3}$ [74] and $1.56 \times 1.56 \times 4 \mathrm{~mm}^{3}=9.75 \mathrm{~mm}^{3}$ [87] than achieved here for $T_{1}$ mapping at voxel sizes of $0.5 \times 0.5 \times 5 \mathrm{~mm}^{3}=1.25 \mathrm{~mm}^{3}$ (single-slice) and $0.75 \times 0.75 \times 5 \mathrm{~mm}^{3}=$ $2.81 \mathrm{~mm}^{3}$ (multi-slice). 

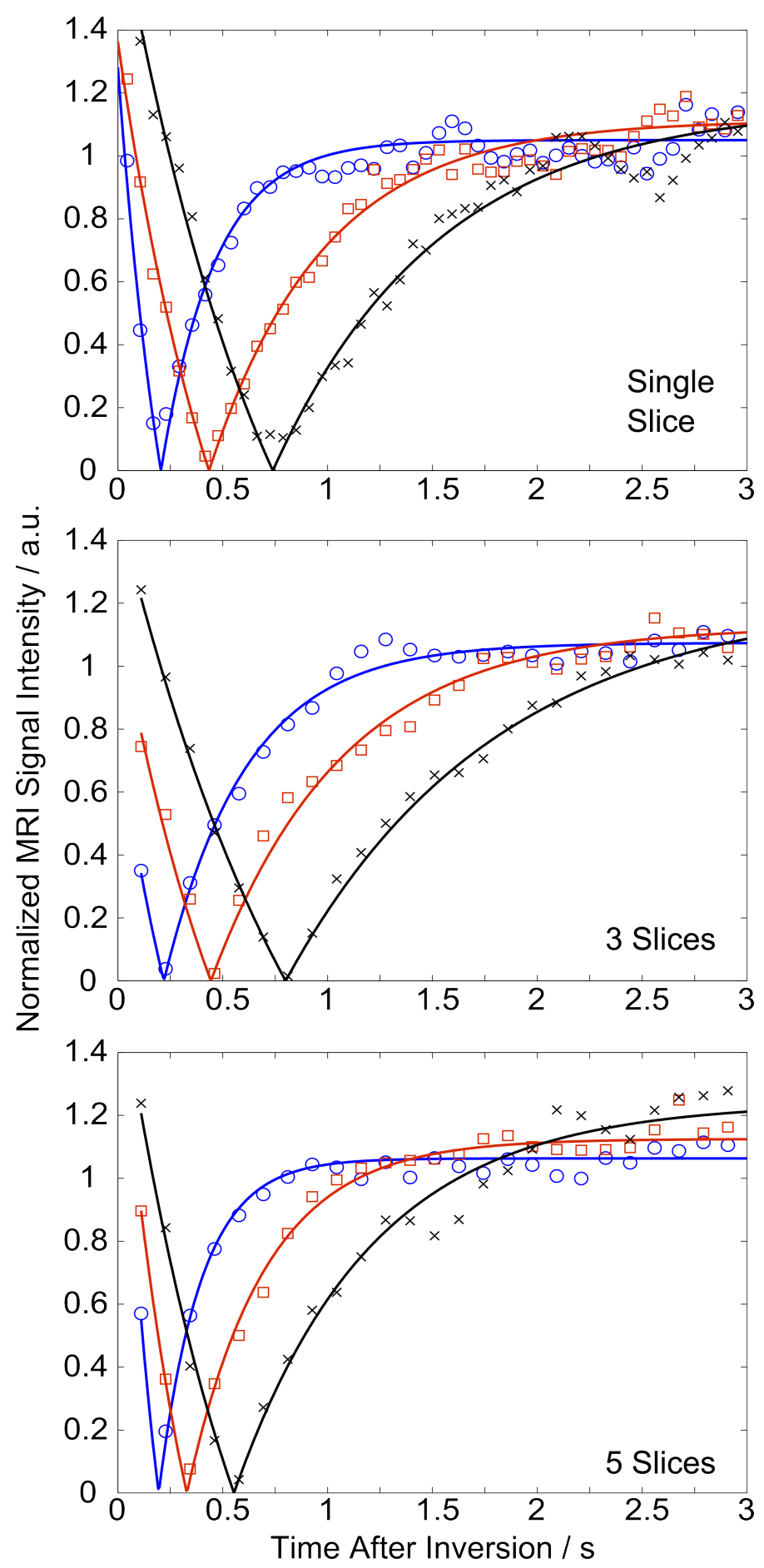

Figure 5.13: Normalized MRI signal intensity time courses and respective fitting results for single pixels of the $T_{1}$ reference phantom (tubes 1,3 and 5 in Figure 5.12 with $T_{1}=0.33 \mathrm{~s}$ (blue), $0.68 \mathrm{~s}$ (red), and $1.25 \mathrm{~s}$ (black). (Top) Single-slice acquisition at $0.5 \mathrm{~mm}$ in-plane resolution, (middle) 3-slice and (bottom) 5-slice acquisition at $0.75 \mathrm{~mm}$ in-plane resolution (same section). 

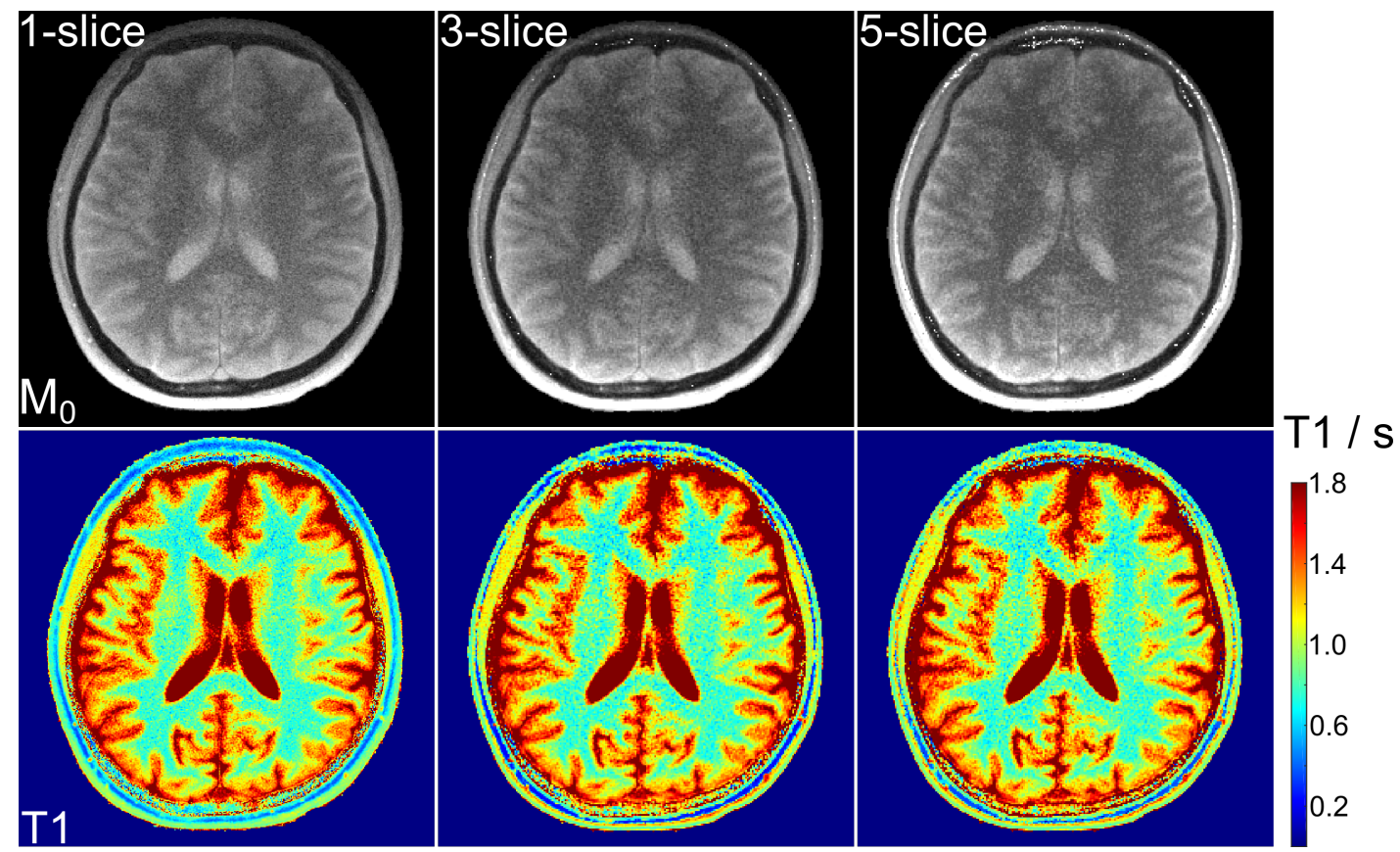

Figure 5.14: Single-shot proton density and $T_{1}$ maps obtained for human brain (healthy subject). (Left) Single-slice acquisition at $0.5 \mathrm{~mm}$ in-plane resolution, (middle) 3-slice and (Right) 5-slice acquisition at 0.75 $\mathrm{mm}$ in-plane resolution (same section). For experimental details see Table 5.4 .

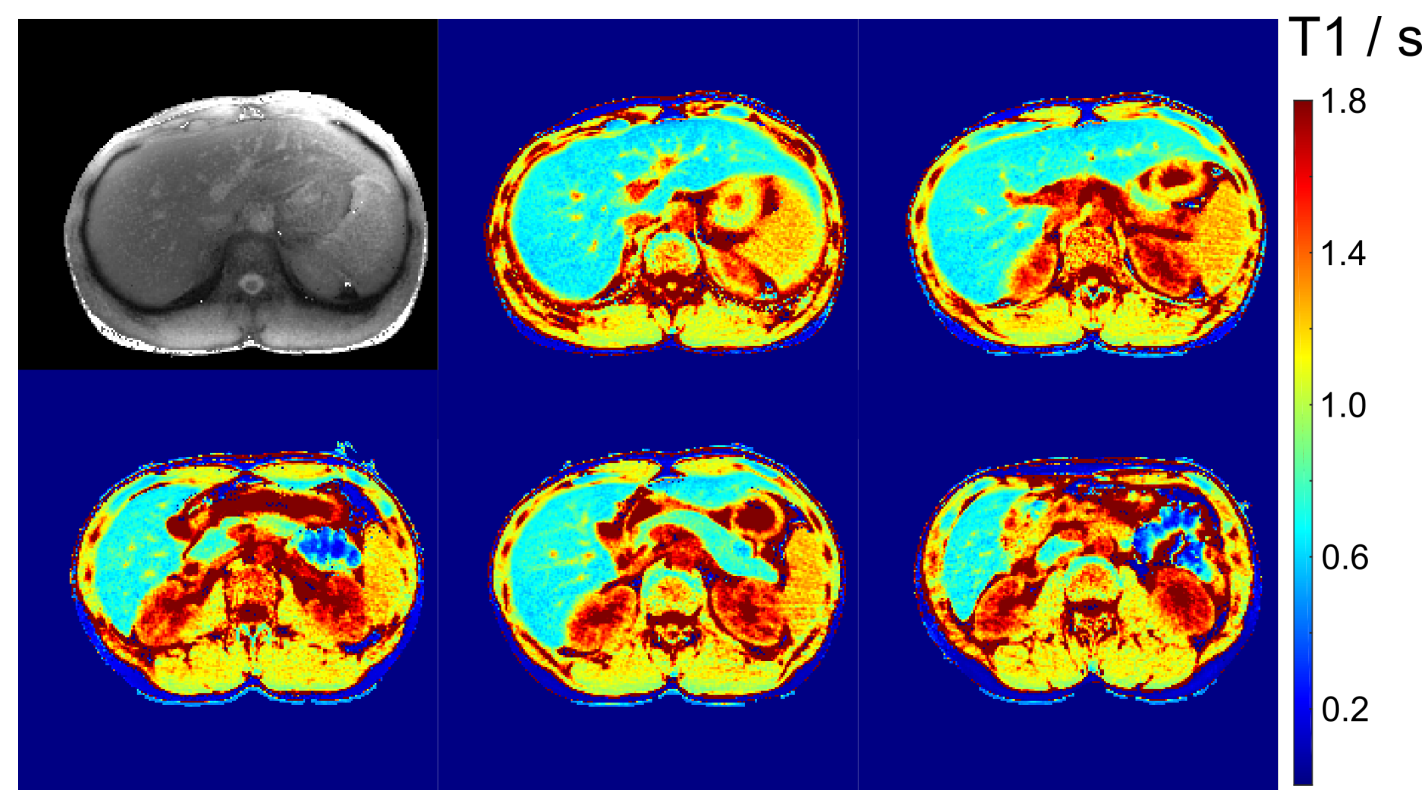

Figure 5.15: (Top left) Single-shot proton density (first section) and (top middle to bottom right) simultaneous multi-slice $T_{1}$ maps at $1.5 \mathrm{~mm}$ in-plane resolution obtained for human abdomen studies. For experimental details see Table 5.4 . 


\subsection{Summary}

In summary, a novel single-shot Look-Locker $T_{1}$ mapping technique based on radial undersampling and NLINV based image reconstruction is proposed. The application to different organ systems demonstrates considerable potential, but certainly awaits larger clinical trials. Moreover, the entire $T_{1}$ mapping procedure including image reconstruction together with denoising and pixel-wise fitting has been integrated into the pipeline of a commercial MRI system. The technical solution allows fast evaluations of $T_{1}$ maps with a minimal delay of several seconds after acquisition and is an important step towards routine clinical applications. 



\section{Single-shot Myocardial T1 Mapping by Real-time MRI}

\subsection{Introduction}

Tissue characterization by native myocardial $T_{1}$ mapping as well as quantitation of perfusion and extracellular volume after contrast administration are essential ingredients of CMR investigations and commonly performed by inversion-recovery (IR) methods using FLASH [64, 88], EPI [89] or SSFP [66] according to the Look-Locker technique [62]. To date, respective applications commonly rely on a modified Look-Locker inversion (MOLLI) experiment [90] or manifold derivatives therefrom - for a recent review of possibilities and limitations see [91]. In fact, despite widespread usage, most approaches still suffer from practical restrictions such as limited spatial resolution and/or compromised $T_{1}$ accuracy, so that further technical improvements are warranted.

Following the recommendations of the $T_{1}$ mapping Consensus Statement of the Society for Cardiovascular Magnetic Resonance and CMR Working Group of the European Society of Cardiology [92], the basic requirements and clinical needs for cardiac $T_{1}$ mapping comprise (i) speed, i.e., single-shot applications with measuring times of a few seconds only, (ii) $T_{1}$ accuracy, i.e., validated $T_{1}$ values with small standard deviations and without dependency on heart rate, (iii) sufficiently high spatial resolution, i.e., about $1 \mathrm{~mm}$ in-plane resolution, and (iv) practical robustness, i.e., no motion sensitivity and no image artifacts due to susceptibility problems, SSFP bandings or radial streakings.

This chapter extends the basic $T_{1}$ mapping approach presented in chapter 5 . It is based on a single-slice acquisition during a brief breathhold (typically 3 s only) which combines a single-shot IR-FLASH technique with pronounced radial undersampling of individual frames, iterative reconstruction as previously described [81, 93], and fitting of a diastolic $T_{1}$ map after deletion of systolic (i.e., motionaffected) frames. The entire procedure is fully automatic and only requires triggering of the initial inversion pulse to early diastole. The results comprise both an image series representing the entire IR experiment and a color-coded $T_{1}$ map where pixel intensities directly refer to $T_{1}$ values in millisecond. The proposed 
$T_{1}$ mapping method complements previous real-time MRI acquisitions of cardiac function and flow [49, 77, 94-96] which together bear the potential to develop a comprehensive real-time CMR examination.

\subsection{Methods}

All measurements were performed on a human MRI system operating at $3 \mathrm{~T}$ (Magnetom Prisma fit, Siemens Healthcare, Erlangen, Germany). Phantom measurements employed the 64-channel head coil, while human heart studies were performed with use of the 18-element thorax coil in combination with 12 elements of the 32-element spine coil. Six young subjects ( 2 female, 4 male, age range 24 to 27 years) with no known illness (heart rate about 50 to $55 \mathrm{bpm}$ ) were recruited among the students of the local university. Written informed consent, according to the recommendations of the local ethics committee, was obtained from all subjects prior to MRI.

According to the $T_{1}$ mapping Consensus Statement [92] experimental validations of the proposed technique were performed at different simulated heart rates with use of a commercial reference phantom (Diagnostic Sonar LTD, Scotland, UK) consisting of 6 compartments with defined $T_{1}$ values surrounded by water. As suggested a long-TR IR fast spin-echo (FSE) sequence with 13 logarithmically spaced inversion times between 50 and 2300 ms served for $T_{1}$ determination (TR $=7.2 \mathrm{~s}, \mathrm{TE}=12 \mathrm{~ms}, 6$ echoes, measuring time $=50 \mathrm{~min}$ ).

The procedures for myocardial $T_{1}$ mapping described below (i.e., data acquisition, image reconstruction and $T_{1}$ fitting) were implemented as an easy-to-use protocol on the MRI system by taking advantage of a bypass computer (sysGen/TYAN Octuple-GPU, Sysgen, Bremen, Germany) previously developed for real-time MRI [49] and equipped with 8 graphical processing units (GeForce GTX, TITAN, NVIDIA, Santa Clara, CA). This bypass computer could be fully integrated into the reconstruction pipeline of the commercial MRI system (Magnetom Prisma fit, Siemens Healthcare, Erlangen, Germany) by a single network connection. If the system software is compatible the implementation takes less than an hour including installation of ready-to-use measuring protocols for cardiac $T_{1}$ mapping and other real-time CMR applications.

\subsubsection{MRI Acquisition and Reconstruction}

The chosen acquisition scheme for myocardial $T_{1}$ mapping is illustrated in Figure 6.1. In order to achieve maximum robustness and $T_{1}$ accuracy, a previously developed IR FLASH sequence [81] was applied as a single-slice technique us- 


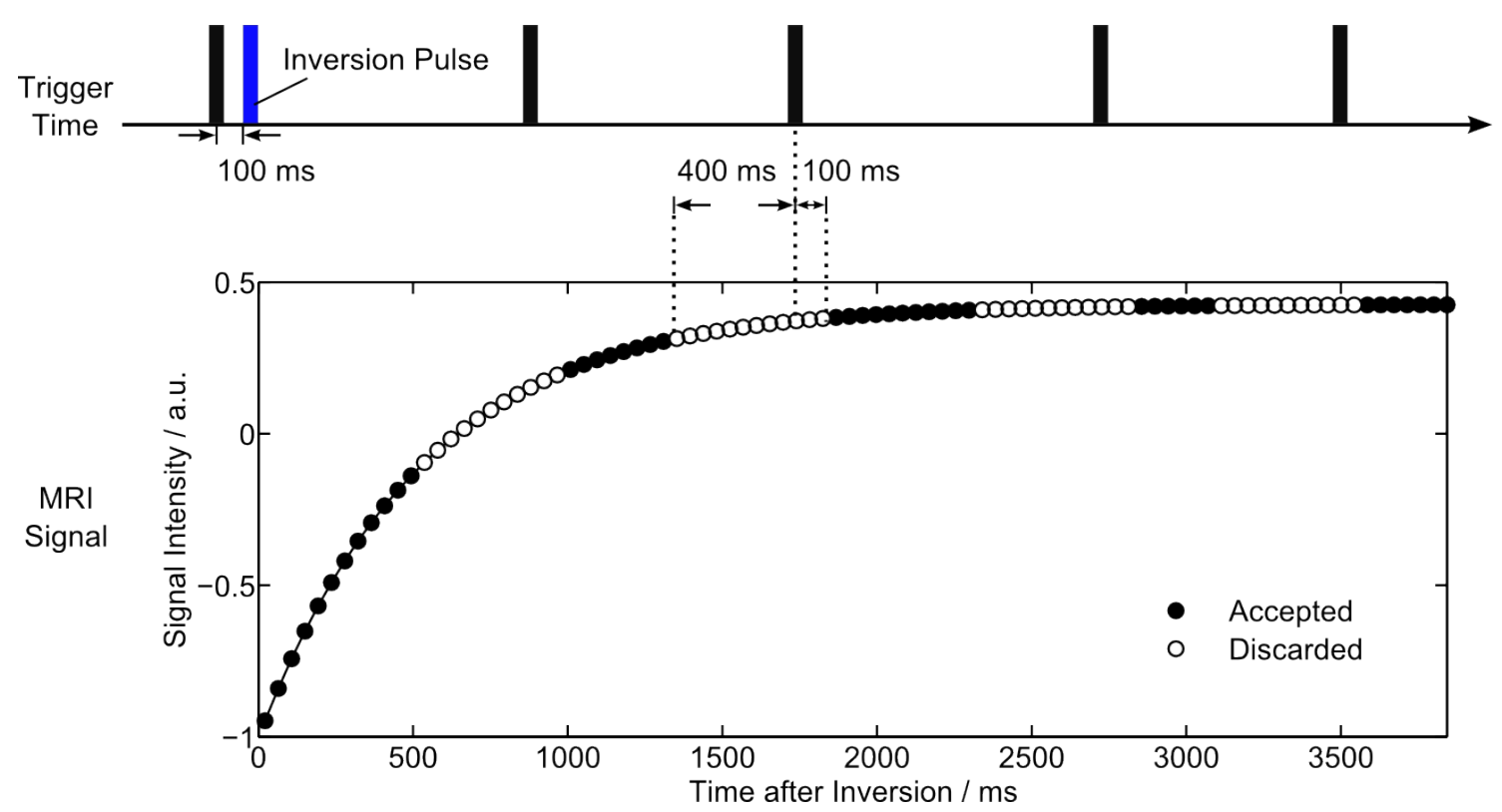

Figure 6.1: Myocardial $T_{1}$ mapping using single-shot IR-FLASH with radial undersampling. Finger pulse trigger $=$ black bar.

ing a non-selective adiabatic $180^{\circ}$ inversion pulse triggered to early diastole. The present study employed a simple and robust finger pulse trigger and a $100 \mathrm{~ms}$ delay to inversion. Although the method yields similar accuracy for a slice-selective inversion pulse when applied to stationary tissue (data not shown), myocardial $T_{1}$ mapping exclusively used a nonselective inversion pulse to minimize the effects of through-plane motion and myocardial perfusion.

Continuous image readout after inversion was based on a radial FLASH sequence with pronounced undersampling. Time-efficient spoiling of residual transverse magnetizations was accomplished by random RF phases [78]. Myocardial $T_{1}$ maps were then acquired at a nominal in-plane resolution of $1.0 \times 1.0 \mathrm{~mm}^{2}$ and $6 \mathrm{~mm}$ section thickness using a FOV $=256 \times 256 \mathrm{~mm}^{2}$ in combination with a resolution of 512 complex data points per radial spoke (using twofold oversampling). All spokes were homogeneously distributed over $360^{\circ}$, while 5 successive frames used complementary sets of spokes in interleaved order. Other parameters were $\mathrm{TR}=2.26 \mathrm{~ms}, \mathrm{TE}=1.47 \mathrm{~ms}$, and flip angle $4^{\circ}$. The number of spokes per frame varied from 27 to 23 and finally 19 spokes yielding a temporal resolution of 61 $\mathrm{ms}, 52 \mathrm{~ms}$ and $43 \mathrm{~ms}$, respectively. The total acquisition time was initially chosen to be $8 \mathrm{~s}$, but later reduced to $4 \mathrm{~s}$ and $3 \mathrm{~s}$.

Image reconstruction has previously been described for the case of non-cardiac $T_{1}$ mapping [81, 93] and employs the same iteratively regularized NLINV algorithm as developed for real-time MRI, for details see [49]. Apart from an advanced gradient-delay correction [95] and data compression to 10 virtual channels based on a principal component analysis, the method takes advantage of some degree 
of spatial smoothness of coil sensitivities as well as of temporal regularization to the preceding frame. However, this latter term of the underlying cost function is downsized relative to the data consistency term by a factor of two during each iteration. The reconstruction ensures high temporal fidelity as demonstrated for a motion phantom rotating at defined speed [97] and therefore does not compromise the resolution of contrast changes during inversion recovery.

The actual reconstruction process started immediately after the end of data acquisition, first by a reverse NLINV reconstruction [49] of the last 10 frames to obtain high-quality coil sensitivity maps using 6 Newton steps. Subsequently, the entire image series was reconstructed in reverse order by fixing the coil sensitivities to those obtained by NLINV. The resulting linear inverse problem was solved by the iteratively regularized conjugate gradient method.

Prior to $T_{1}$ fitting the images were spatially filtered by a recently developed modified non-local means algorithm [54] which has been briefly introduced in section 5.4.1 for precision improvement.

Temporal median filtering was applied pixelwise and only used for the purpose of displaying image series, whereas no temporal filter was used for $T_{1}$ mapping. The median filter extended over 5 frames to match the number of frames with different sets of spokes, e.g. see [49, 97]. As illustrated in Figure 6.1, the influence of systolic motion on the fitting of a diastolic $T_{1}$ map was minimized by automatically deleting images over a period of $500 \mathrm{~ms}$ starting from $400 \mathrm{~ms}$ prior to each finger pulse trigger signal.

\subsubsection{T1 Quantitation}

After reconstruction, spatial filtering and systolic deletion the remaining complex images were fitted to the complex signal model

$$
M(t)=M_{0}\left(\gamma-(1+\gamma) e^{-t / T_{1}^{*}}\right)
$$

where $M_{0}$ is the initial complex signal after inversion, $\mathrm{t}$ is the central time point (i.e., radial spoke) of each frame during inversion recovery, $\gamma$ is the ratio between the steady-state signal $M_{s s}$ and $M_{0}$, and $T_{1}^{*}$ is the shortened apparent $T_{1}$ due to multiple low flip-angle RF excitations. The same phase is assumed for $M_{s s}$ and $M_{0}$, which leads to four unknown real-valued parameters: $\Re\left\{M_{0}\right\}, \Im\left\{M_{0}\right\}, \gamma$ and $T_{1}^{*}$. A pixelwise estimation was performed using the Trust-Region algorithm (Chapter 4.1 and 4.3 in [23]) based on the Dlib $\mathrm{C}++$ library [98]. The algorithm performs 
an unconstrained minimization of the cost function defined by

$$
\frac{1}{2} \sum_{t}\left(\Re\{M(t)-y(t)\}^{2}+\Im\{M(t)-y(t)\}^{2}\right)
$$

where $y(t)$ is the corresponding pixel intensity at time $t$ during inversion recovery. The iterative optimization was stopped if the relative difference of the objective function values between successive iterations was less than $10^{-5} \cdot T_{1}$ was then calculated by [99]:

$$
T_{1}=T_{1}^{*} / \gamma+2 \delta t
$$

with $\delta t$ the delay time between the center of inversion pulse and the start of data acquisition. In the present implementation this period covered half of the inversion pulse $(5 \mathrm{~ms})$ and a following spoiler gradient $(10 \mathrm{~ms})$. For the assessment of myocardial $T_{1}$ values in the septal wall, the regions of interest were carefully selected to exclude the blood pool. These analyses were accomplished using the arrayShow tool [79] in MATLAB (MathWorks, Natik, MA).

Table 6.1: $T_{1}$ relaxation times for a reference phantom and simulated heart rates. ${ }^{1}$

\begin{tabular}{ccccccc}
\hline$T_{1} / \mathrm{ms}^{2}$ & $331 \pm 11$ & $494 \pm 22$ & $676 \pm 19$ & $857 \pm 25$ & $1225 \pm 20$ & $1501 \pm 23$ \\
$T_{2} / \mathrm{ms}^{3}$ & $101 \pm 2$ & $46 \pm 2$ & $81 \pm 2$ & $132 \pm 5$ & $138 \pm 5$ & $166 \pm 5$ \\
\hline Heart rate $^{4}$ & & & & & & \\
\hline 0 & $315 \pm 13$ & $476 \pm 18$ & $660 \pm 25$ & $850 \pm 28$ & $1227 \pm 34$ & $1511 \pm 42$ \\
40 & $315 \pm 13$ & $475 \pm 18$ & $659 \pm 26$ & $850 \pm 28$ & $1226 \pm 34$ & $1513 \pm 43$ \\
60 & $314 \pm 13$ & $475 \pm 18$ & $661 \pm 27$ & $852 \pm 30$ & $1230 \pm 36$ & $1513 \pm 44$ \\
80 & $316 \pm 13$ & $476 \pm 20$ & $663 \pm 29$ & $853 \pm 30$ & $1230 \pm 37$ & $1516 \pm 46$ \\
100 & $317 \pm 16$ & $479 \pm 23$ & $665 \pm 31$ & $853 \pm 35$ & $1236 \pm 38$ & $1517 \pm 50$ \\
\hline
\end{tabular}

${ }^{1}$ Single-shot IR FLASH was performed at $43 \mathrm{~ms}$ resolution (19 spokes) for a duration of $3 \mathrm{~s}$.

${ }^{2} T_{1}$ values for a long-TR IR-FSE sequence.

${ }^{3} T_{2}$ values according to Ref. [28].

${ }^{4}$ Simulated heart rates (in beats per minute) correspond to the deletion of a $500 \mathrm{~ms}$ period ("systole") in each cardiac cycle. No images are deleted for zero heart rate.

\subsection{Results}

Table 6.1 summarizes $T_{1}$ relaxation times for a reference phantom. The data were acquired with the radial IR-FLASH method proposed for myocardial $T_{1}$ mapping (43 ms resolution, $3 \mathrm{~s}$ duration) at different simulated heart rates ranging from 40 to $100 \mathrm{bpm}$. Zero heart rate refers to $T_{1}$ fitting without deletion of any frames. A comparison with $T_{1}$ relaxation times obtained by a long-TR IR-FSE technique 
reveals excellent agreement for most (long) values, while two tubes with shorter values are slightly underestimated (maximum deviation 5\%). Figures 6.2 and 6.3 demonstrate myocardial $T_{1}$ maps for different numbers of spokes per frame, i.e. different temporal resolution, and different durations of the IR-FLASH measurement, respectively. In all cases, visual inspection reveals no detectable difference. This qualitative finding is confirmed by the quantitative analysis in Table 6.3. The effect of filtering prior to $T_{1}$ fitting is demonstrated in Figure 6.4 comparing raw images and $T_{1}$ maps with and without application of a spatial filter. Figure 6.5 shows three $T_{1}$ maps for a single subject in a basal, mid-ventricular and apical short-axis section, while Figure 6.6 summarizes the mid-ventricular $T_{1}$ maps of all 6 subjects. The quantitative results for all 6 subjects are summarized in Table 6.3 (septal $T_{1}$ values in a basal, mid-ventricular and apical section) and Table 6.4 (segmental $T_{1}$ values in a mid-ventricular section), respectively.

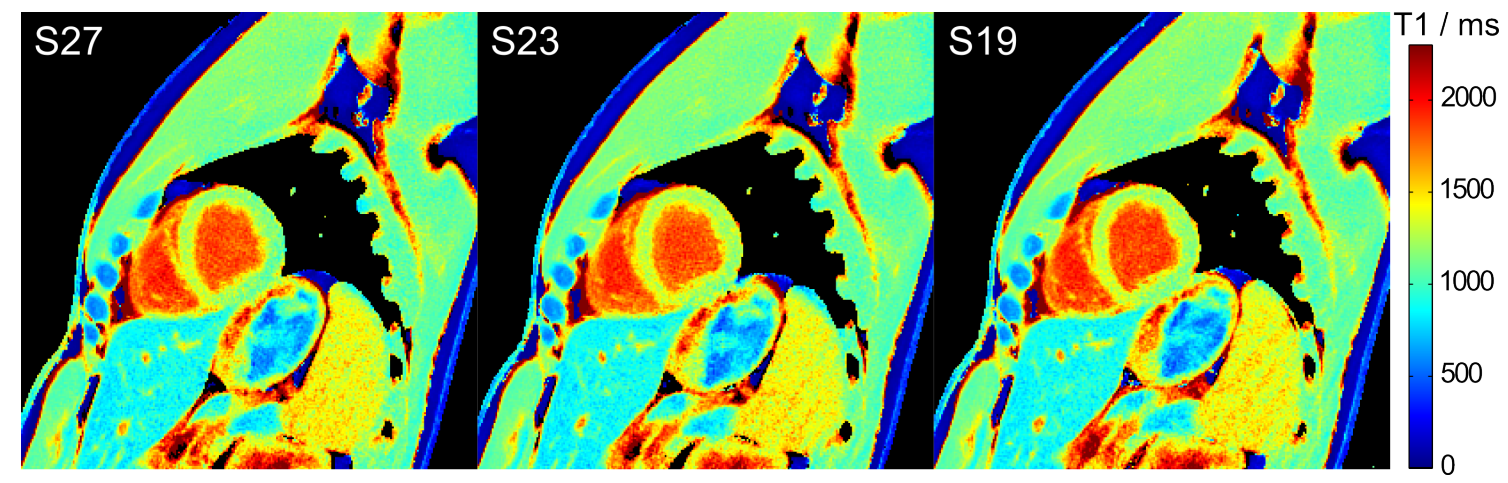

Figure 6.2: Myocardial $T_{1}$ maps for different temporal resolutions. Single-shot IRFLASH was performed with 27, 23 and 19 spokes per frame (i.e., acquisition times of 63,52 and $43 \mathrm{~ms}$ ) for a duration of $8 \mathrm{~s}$.

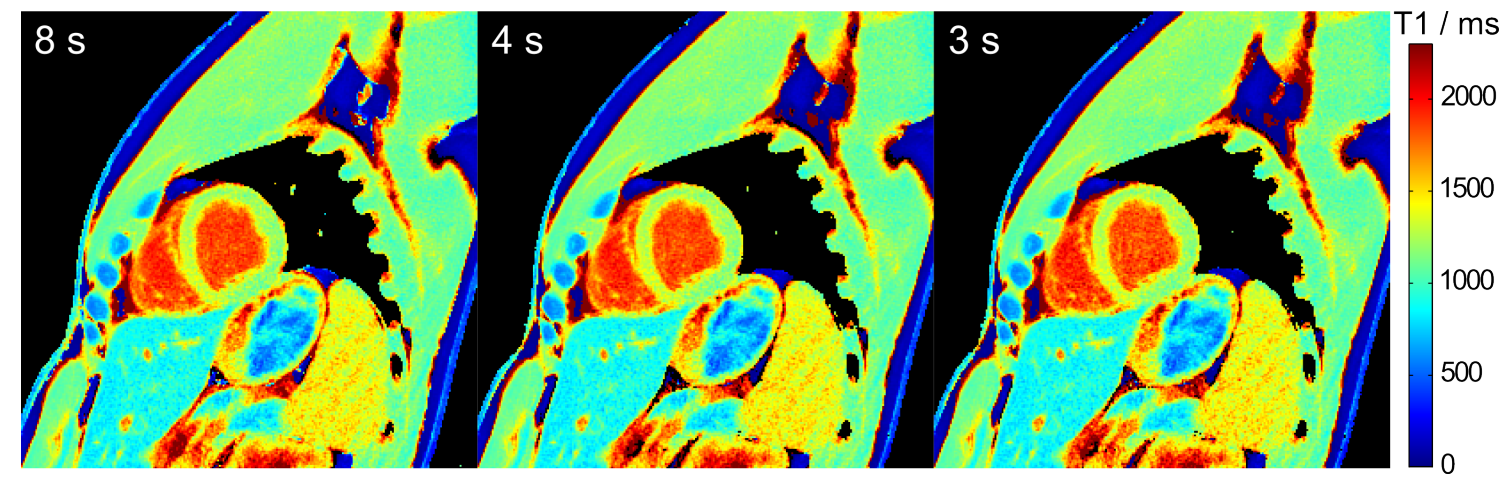

Figure 6.3: Myocardial $T_{1}$ maps for different measurement durations. Single-shot IR-FLASH was performed at $43 \mathrm{~ms}$ temporal resolution (19 spokes) for durations of $8 \mathrm{~s}, 4 \mathrm{~s}$, and $3 \mathrm{~s}$. 
Table 6.2: Mean $T_{1}$ values ${ }^{1}$ of the mid-ventricular septal wall for different temporal resolutions ${ }^{2}$ and durations ${ }^{3}$.

\begin{tabular}{cccc}
\hline $\begin{array}{c}\text { Spokes per frame } \\
\text { Resolution / ms }\end{array}$ & 27 & 23 & 19 \\
\hline IR FLASH & & 52 & 43 \\
\hline $8 \mathrm{~s}$ & $1253 \pm 29$ & $1254 \pm 29$ & $1253 \pm 31$ \\
$4 \mathrm{~s}$ & $1252 \pm 30$ & $1254 \pm 30$ & $1253 \pm 31$ \\
$3 \mathrm{~s}$ & $1254 \pm 32$ & $1257 \pm 33$ & $1256 \pm 33$ \\
\hline
\end{tabular}

${ }^{1}$ Averaged across subjects (mean $\pm S D, n=6$ ).

${ }^{2}$ Number of spokes and acquisition time per frame.

${ }^{3}$ Measuring time of single-shot IR-FLASH.

Table 6.3: $T_{1}$ relaxation times of the septal wall.

\begin{tabular}{|c|c|c|c|}
\hline Subject & $\begin{array}{c}\text { Basal } \\
T_{1} / \sharp \text { Frames }^{1}\end{array}$ & $\begin{array}{c}\text { Mid-ventricular } \\
T_{1} / \sharp \text { Frames }\end{array}$ & $\begin{array}{c}\text { Apical } \\
T_{1} / \sharp \text { Frames }\end{array}$ \\
\hline 1 & $1250 \pm 69 / 48$ & $1256 \pm 64 / 48$ & $1298 \pm 72 / 38$ \\
\hline 2 & $1237 \pm 60 / 48$ & $1263 \pm 54 / 47$ & $1291 \pm 58 / 47$ \\
\hline 3 & $1270 \pm 74 / 35$ & $1287 \pm 69 / 35$ & $1332 \pm 60 / 38$ \\
\hline 4 & $1277 \pm 71 / 47$ & $1295 \pm 61 / 49$ & $1298 \pm 50 / 47$ \\
\hline 5 & $1215 \pm 68 / 49$ & $1209 \pm 51 / 47$ & $1256 \pm 48 / 47$ \\
\hline 6 & $1227 \pm 66 / 49$ & $1227 \pm 51 / 47$ & $1253 \pm 46 / 48$ \\
\hline Mean $^{2}$ & $1246 \pm 24$ & $1256 \pm 33$ & $1288 \pm 30$ \\
\hline Ref.[100] & 1157 & 1159 & 1181 \\
\hline Ref.[101] & & 1315 & \\
\hline Ref.[102] & & 1286 & \\
\hline
\end{tabular}

${ }^{1} T_{1}$ (in ms, mean \pm SD in a ROI covering most of the septal wall) for single-shot IR-FLASH at $43 \mathrm{~ms}$ resolution (19 spokes) and $3 \mathrm{~s}$ duration. $\sharp$ Frames refers to the number of images retained after deletion of systolic frames.

${ }^{2}$ Mean \pm SD across subjects. 
(a)

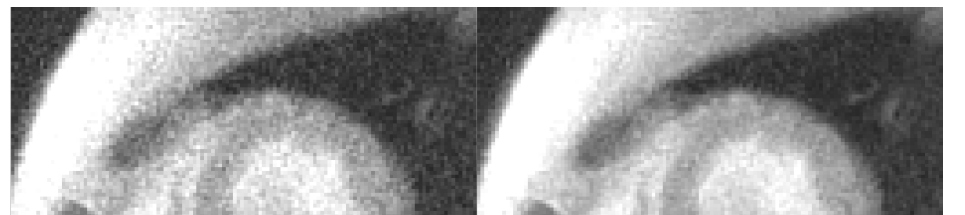

(b)

(c)

(d)

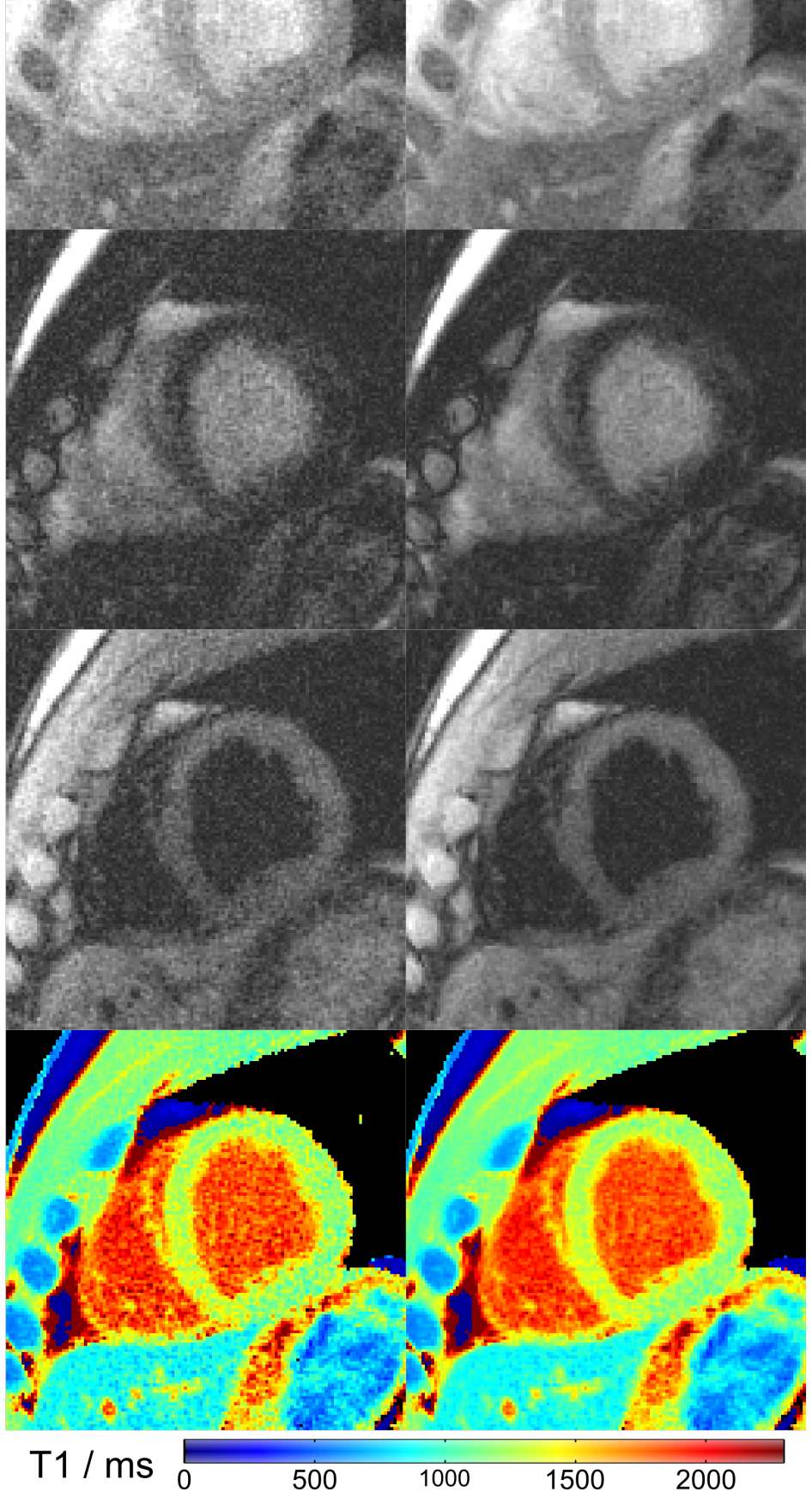

Figure 6.4: Cardiac images (a-c) and $T_{1}$ maps (d) (left) without and (right) with spatial filtering. Single-shot IR-FLASH was performed at $43 \mathrm{~ms}$ temporal resolution (19 spokes) for a duration of $3 \mathrm{~s}$. The images (magnified views) refer to an early time point after inversion (a), nulling of myocardial tissue (b), and nulling of blood signal (c), respectively. 


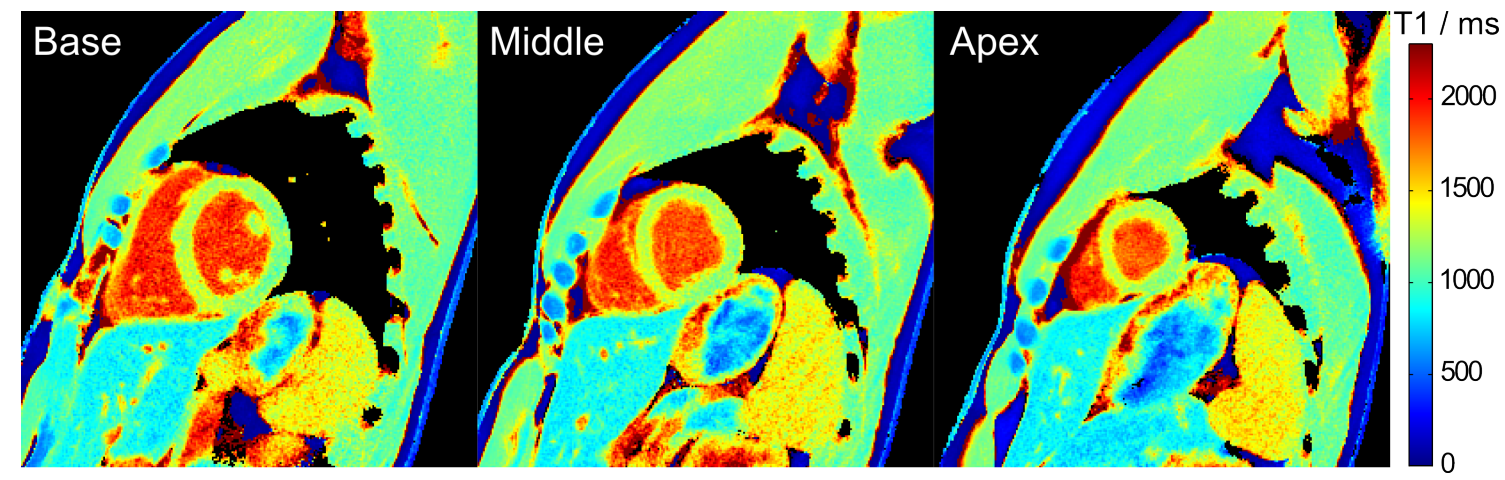

Figure 6.5: Myocardial $T_{1}$ maps of a basal, mid-ventricular and apical section. Single-shot IR-FLASH was performed at $43 \mathrm{~ms}$ resolution (19 spokes) for a duration of $3 \mathrm{~s}$.

Table 6.4: Regional myocardial $T_{1}$ relaxation times ${ }^{1}$.

\begin{tabular}{ccccc}
\hline Subject & Anterior & Septal & Inferior & Lateral \\
\hline 1 & $1295 \pm 70$ & $1261 \pm 70$ & $1223 \pm 95$ & $1218 \pm 70$ \\
2 & $1191 \pm 60$ & $1259 \pm 56$ & $1245 \pm 91$ & $1157 \pm 64$ \\
3 & $1212 \pm 62$ & $1291 \pm 68$ & $1270 \pm 89$ & $1238 \pm 69$ \\
4 & $1224 \pm 64$ & $1304 \pm 64$ & $1230 \pm 92$ & $1217 \pm 68$ \\
5 & $1169 \pm 56$ & $1206 \pm 59$ & $1155 \pm 79$ & $1166 \pm 57$ \\
6 & $1173 \pm 56$ & $1234 \pm 58$ & $1171 \pm 66$ & $1156 \pm 69$ \\
\hline Mean $^{2}$ & $1211 \pm 47$ & $1259 \pm 36$ & $1288 \pm 30$ & $1192 \pm 36$ \\
\hline
\end{tabular}

${ }^{1} T_{1}$ (in ms, mean \pm SD per standardized ROI in a midventricular section) for single-shot IR-FLASH at $43 \mathrm{~ms}$ resolution (19 spokes) and $3 \mathrm{~s}$ duration.

${ }^{2}$ Mean \pm SD across subjects. 


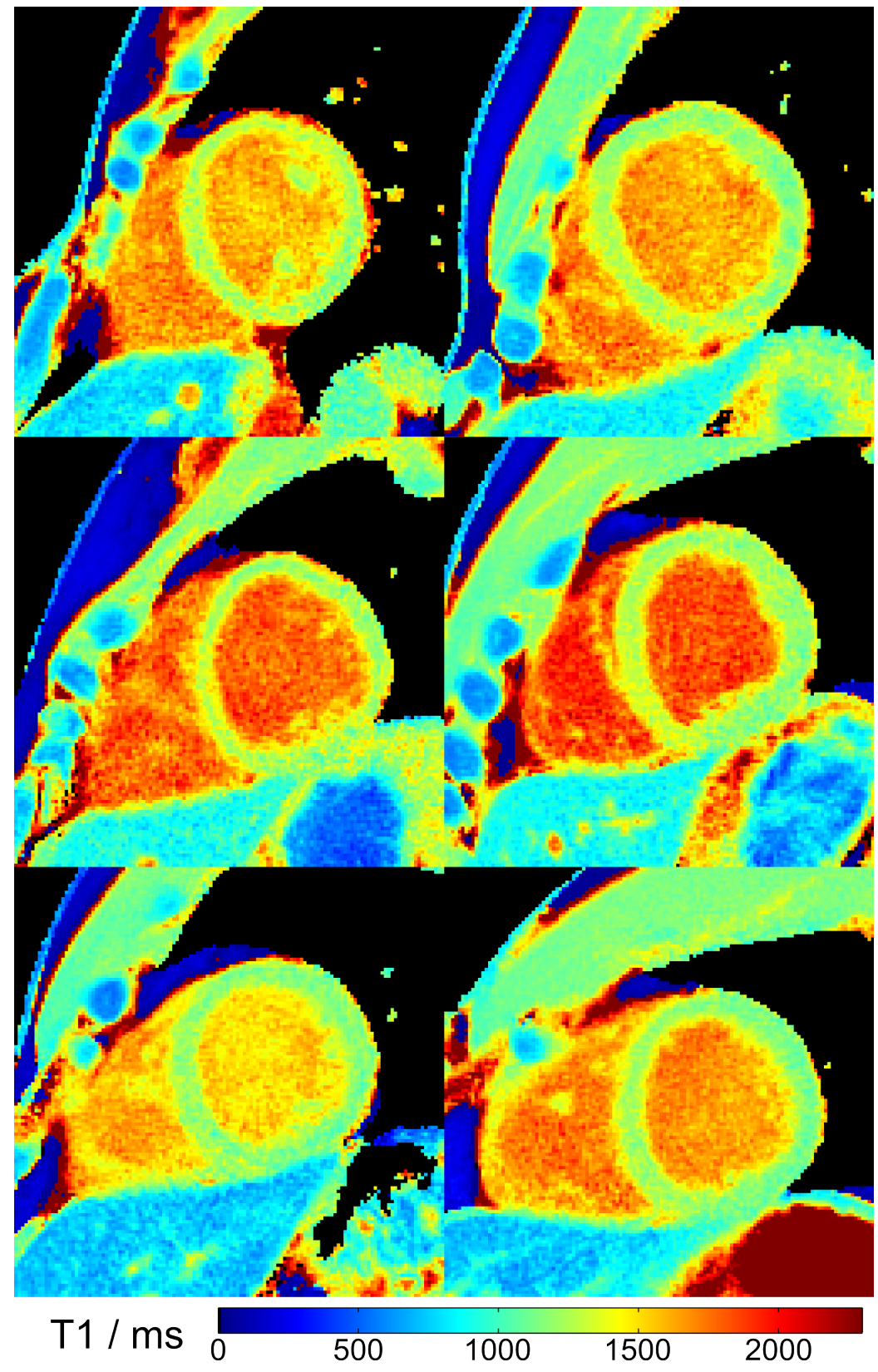

Figure 6.6: Myocardial $T_{1}$ maps of all 6 subjects (mid-ventricular section, magnified view). Single-shot IR-FLASH was performed at $43 \mathrm{~ms}$ resolution (19 spokes) for a duration of $3 \mathrm{~s}$. 


\subsection{Discussion}

This chapter describes a novel method for myocardial $T_{1}$ mapping which offers accuracy, high spatial resolution, practical robustness and speed. The results indicate that myocardial $T_{1}$ mapping may be performed at a nominal resolution of $1.0 \mathrm{~mm}$ with a temporal resolution of $43 \mathrm{~ms}$ per frame using a short IR-FLASH measurement of only $3 \mathrm{~s}$.

$T_{1}$ accuracy was confirmed in a phantom study providing reference $T_{1}$ values for a long-TR IR-FSE sequence. A slight underestimation of $T_{1}$ relaxation times for two tubes with values of about 500 and $675 \mathrm{~ms}$ coincides with the occurrence of the shortest $T_{2}$ relaxation times of 46 and $81 \mathrm{~ms}$, respectively (compare Table 6.1). The slight deviation of $T_{1}$ values may therefore be due to a partial failure of the "reference" IR-FSE acquisition which extends to a maximum echo time of $72 \mathrm{~ms}$ and thus may affect the image definition of signal contributions with short $T_{2}$ relaxation times. Similar effects are to be expected for IR methods with a SSFP readout module, because such sequences require relatively long $T_{2}$ relaxation times to build up sufficiently strong transverse coherences.

Apart from $T_{1}$ accuracy, the results in Table 6.1 confirm the independence of $T_{1}$ quantitation on the heart rate up to $100 \mathrm{bpm}$ which effectively refers to the independence of $T_{1}$ on the number of fitted images after elimination of "systolic" frames. This advantageous behavior reflects the fact that the highly undersampled radial FLASH readout ensures a sufficiently large number of frames for a proper sampling of the IR signal time course. $T_{1}$ precision was also demonstrated to be high both in vitro and in vivo by small SD values of $3-5 \%$ of the mean for phantom measurements and $4-8 \%$ for septal $T_{1}$ values (compare Tables 6.1 to 6.4). In addition, the comparison in Figure 6.4 indicates that the achieved $T_{1}$ mapping quality not necessarily depends on the use of filtering. Nevertheless, while highquality $T_{1}$ maps may be obtained by fitting unfiltered images, the use of a newly modified non-local means filter [54] further improves the SNR of $T_{1}$ maps without the expense of blurring.

Although myocardial $T_{1}$ relaxation times found here were in general agreement with literature values, comparisons to previous results are compromised by numerous technical differences or even inadequacies. As an example, the current values $(1246-1288 \mathrm{~ms})$ are in the range of those reported in [102] (1286 ms), but slightly higher than in [100] (1157 - 1181 ms) and lower than in [101] (1315 ms), who all used similar MOLLI sequences. Of course, all techniques including the one proposed here suffer from some general limitations of the Lock-Locker approach which often are due to the presence of residual motion both in plane and through plane. For example, diastolic circulation of blood within the ventricles leads to im- 
age intensities which violate the expected IR signal model and preclude a reliable fitting of blood $T_{1}$ relaxation times. Even myocardial movements may play a role during early diastolic phases. However, this mainly becomes a problem for motionsensitive $T_{1}$ such as SSFP sequences, whereas short-TE FLASH sequences as used here and recently proposed by others [103, 104] are much less affected. This is because SSFP sequences inherently rely on the establishment of phase coherence over multiple repetition intervals which is commonly precluded (i.e., spoiled) in the presence of motion, whereas FLASH sequences interrogate a pool of longitudinal magnetization with independent low-flip angle excitations that give rise to spin-density weighted images. In fact, when exploiting the additional motion robustness of radial encodings in the present implementation, preliminary trials of myocardial $T_{1}$ mapping during free breathing showed little if any qualitative and quantitative difference to breathhold scans (data not shown). Thus, the proposed method seems to be robust enough to even work in patients who are unable to perform any breathing protocol.

Another factor contributing to myocardial $T_{1}$ values is the different access to high spatial resolution and the concomitant consideration of partial volume effects. Such problems have been reported for thin myocardial walls [91] including the assessment of fibrosis in the peri-infarct zone as well as for the right ventricle [105]. These $T_{1}$ mapping studies using MOLLI techniques were performed at $1.4 \times 1.9 \times 8.0 \mathrm{~mm}^{3}$ for low heart rates and $1.9 \times 2.3 \times 8.0 \mathrm{~mm}^{3}$ at high heart rates [91]. A higher resolution of $1.2 \times 1.2 \times 4 \mathrm{~mm}^{3}$ was only achieved with the use of a segmented readout module after inversion which therefore required repetitive acquisitions and very long measuring times of $2.5 \mathrm{~min}$ per $T_{1}$ map [105]. Another recent work using IR-FLASH employed a sliding-window reconstruction [104] at $1.17 \times 1.17 \times 8 \mathrm{~mm}^{3}$ resolution, which was achieved by twofold zero-padding, i.e. an interpolation of the acquired resolution. To the best of our knowledge, the method proposed here is the first technique for myocardial $T_{1}$ mapping which offers $1.0 \times 1.0 \times 6.0 \mathrm{~mm}^{3}$ resolution within a measuring time of only $3 \mathrm{~s}$.

The most important limitation of this study is the small sample size. This is because the work represents a new technical development which requires basic validation with use of a $T_{1}$ reference phantom and a group of normal subjects. Obviously, this precedes any evaluation of the clinical utility of the proposed $T_{1}$ mapping in a large cohort of patients. Moreover, at this stage, widespread clinical applications are hampered by the fact that the technical solution requires dedicated software and hardware which so far is only available for MRI systems of the same manufacturer as used here. A remaining temporary restriction is the computational time needed for image reconstruction and $T_{1}$ fitting which currently takes about $13 \mathrm{~s}$ per $T_{1}$ map. Nevertheless, this may not necessarily block the clinical 
workflow, because a delayed reconstruction does not interfere with continuous acquisitions.

\subsection{Summary}

Myocardial $T_{1}$ mapping based on single-shot IR-FLASH with radial undersampling and iterative reconstruction as well as $T_{1}$ fitting with automated deletion of systolic frames meets most current clinical challenges. The proposed method warrants extensive clinical trials as it promises significant advantages for CMR studies which rely on native or post-contrast $T_{1}$ quantitation. 



\section{Model-based Reconstruction for Single-shot Inversion-recovery Look-Locker T1 Mapping with Sparsity Constraints}

Apart from techniques introduced in chapter 5 for acceleration of inversion-recovery Look-Locker $T_{1}$ mapping, other methods making use of model information have also been proposed for speed-up of parameter mapping in general. One way is to include the underlying model into the image reconstruction process explicitly with some constraints (e.g., sparsity, low-rank or joint sparsity and low-rank) in the parameter dimension [106-111]. After images with different contrasts being reconstructed, a pixelwise fitting is then followed to obtain parameter maps. The other way is to estimate parameter maps directly from undersampled $k$-space data by model-based reconstruction, bypassing the image reconstruction step completely [112-117]. Specifically with respect to single-shot Look-Locker based parameter mapping, it has been proposed to iteratively fit the parameters of the monoexponential model to undersampled radial $k$-space data [76, 87] or to jointly estimate three parameter maps as a solution of a nonlinear inverse problem [? ].

In the following chapter, the general concept in [47, 113] is expanded by formulating the direct parameter estimation from $k$-space as a solution of a nonlinear inversion problem. Both parameter maps and coil sensitivities are simultaneously estimated from the whole inversion recovery radially acquired data. Sparsity constraints are applied to the parameter maps to improve the conditioning of the inverse problem [115]. The above nonlinear inversion problem is then solved by the iteratively regularized Gauss-Newton method (IRGNM) within which the fast iterative shrinkage-thresholding algorithm (FISTA) is employed to solve the $\ell_{1}$ regularized subproblem. The accuracy of the proposed $T_{1}$ mapping method is determined for simulated data and experimentally validated in phantom, human brain and abdomen studies. A comparison to the method presented in chapter 5 is also given. 


\subsection{Theory}

\subsubsection{Signal Equation}

The inversion recovery Look-Locker based sequence consists of a magnetization preparation pulse, i.e., inversion pulse, followed by a series of low flip angle RF excitations with spoiled gradient echo readout. The MRI signal during this process obtained from multiple receiver coils can be described by

$$
s_{j}(t)=\int M(\vec{r}) c_{j}(\vec{r}) e^{-i \vec{r} \cdot \vec{k}(t)} d \vec{r}
$$

with $c_{j}(\vec{r})$ the $j$ th coil, $\vec{r}$ the position in image space, $\vec{k}(t)$ the chosen $k$-space trajectory and $M(\vec{r})$ the magnetization signal at time $t_{k}$ after inversion [62, 64] :

$$
M_{t_{k}}(\vec{r})=M_{s s}(\vec{r})-\left(M_{s s}(\vec{r})+M_{0}(\vec{r})\right) \cdot e^{-t_{k} \cdot R_{1}^{*}(\vec{r})}
$$

where $M_{s s}$ represents the steady-state magnetization, $M_{0}$ is the equilibrium magnetization, $t_{k}$ the inversion time and $R_{1}^{*}$ the effective relaxation rate (i.e., $R_{1}^{*}=$ $1 / T_{1}^{*}$. With an estimation of three parameters $M_{s s}, M_{0}, R_{1}^{*}$ from equation (7.2) and an assumption $\mathrm{TR} \ll T_{1}^{*}, T_{1}$, the desired $T_{1}$ can be calculated according to [62]:

$$
T_{1}=\frac{M_{0}}{M_{s s} \cdot R_{1}^{*}}
$$

\subsubsection{Model-based Reconstruction}

Here, the estimation of both parameter maps and coil sensitivities from $k$-space is formulated as a nonlinear inverse problem, i.e., signal equation (7.1) is understood as a nonlinear equation with an operator $F$ which maps parameters $\left(M_{s s}, M_{0}, R_{1}^{*}\right)^{T}$ and coil sensitivity profiles $\left(c_{1} \ldots c_{N}\right)^{T}$ to the measured $k$-space data $y$ :

$$
F(x)=y \quad \text { with } \quad x=\left(\begin{array}{c}
M_{s s} \\
M_{0} \\
R_{1}^{*} \\
c_{1} \\
\vdots \\
c_{N}
\end{array}\right)
$$


and the forward operator $F$ given by:

$$
F: x \mapsto\left(\begin{array}{c}
P_{1} \mathcal{F}\left\{c_{1} \cdot M\left(M_{s s}, M_{0}, R_{1}^{*}, t_{1}\right)\right\} \\
\vdots \\
P_{1} \mathcal{F}\left\{c_{N} \cdot M\left(M_{s s}, M_{0}, R_{1}^{*}, t_{1}\right)\right\} \\
P_{2} \mathcal{F}\left\{c_{1} \cdot M\left(M_{s s}, M_{0}, R_{1}^{*}, t_{2}\right)\right\} \\
\vdots \\
P_{n} \mathcal{F}\left\{c_{N} \cdot M\left(M_{s s}, M_{0}, R_{1}^{*}, t_{n}\right)\right\}
\end{array}\right)
$$

where $\mathcal{F}$ is the Fourier transformation, $P$ is the orthogonal projection onto the trajectory, $M$ is the model prescribed in equation (7.2) and $n$ is the number of $k$-space frames during inversion recovery. Because the joint reconstruction of parameter maps and coil sensitivities is an ill-posed problem, an optimization of the least square residual $\|F(x)-y\|_{2}^{2}$ would yield an unrealistic solution. To overcome this, additional a priori information (i.e., regularization terms) needs to be incorporated leading to the following optimization problem:

$$
\hat{x}=\underset{x}{\operatorname{argmin}}\|F(x)-y\|_{2}^{2}+\alpha R\left(x_{\mathbf{p}}\right)+\beta Q\left(x_{c}\right) .
$$

Here, $x_{\mathbf{p}}$ represents the three parameter maps, i.e., $x_{\mathbf{p}}=\left(M_{s s}, M_{0}, R_{1}^{*}\right)^{T}, x_{c}$ represents the coils, i.e., $x_{c}=\left(c_{1}, \ldots, c_{N}\right)^{T} . R(\cdot)$ and $Q(\cdot)$ are regularizations on parameter maps and coil sensitivities respectively. $\alpha$ and $\beta$ are regularization parameters. Equation (7.6) is then solved by iteratively regularized Gauss-Newton method (IRGNM] [48, 118], which has been previously introduced for NLINV [47]. The IRGNM iteratively updates an estimate of the solution based on the linearization of the above nonlinear problem:

$$
x_{n+1}=\underset{x}{\operatorname{argmin}}\left\|D F\left(x_{n}\right)\left(x-x_{n}\right)+F\left(x_{n}\right)-y\right\|_{2}^{2}+\alpha_{n} R\left(x_{\mathbf{p}}\right)+\beta_{n} Q\left(x_{c}\right)
$$

where $D F\left(x_{n}\right)$ is the Fréchet derivative (or Jacobian) of $F$ at the point $x_{n}$ and regularization parameters $\alpha_{n}, \beta_{n}$ are reduced in each iteration step, i.e., $\alpha_{n}=\alpha_{0} \cdot q_{\alpha}^{n}$ and $\beta_{n}=\beta_{0} \cdot q_{\beta}^{n}$ with $q_{\alpha}, q_{\beta} \in(0,1)$. Under certain conditions the iterative updates of $x_{n+1}$ will converge to a solution.

\subsubsection{Regularization}

The model-based reconstruction adopts the $L^{2}$-norm of weighted Fourier coefficients of sensitivities in [47] to enforce the intrinsic smoothness of coil sensitivities. For the parameter maps Tikhonov regularizations can be employed with which very efficient optimization algorithms such as the CG method can be used. 
However, as $\alpha_{n}$ is decreasing during the iterations, the problem in equation (7.7) will become increasingly ill-conditioned. This leads to noise amplification in the later Gauss-Newton steps, which can be counteracted by replacing the $L^{2}$ penalty with other regularizations, e.g., total variation (TV) or total generalized variation (TGV) [119]. One drawback of this type of regularization is that it usually entails staircase artifacts which is not favorable to the clinicians. Hence a wavelet-based $\ell_{1}$ regularization is used instead to penalize the parameter maps. By defining $w_{r p}$ the wavelet coefficient of the $p$ th parameter map at the spatial position $r$, the $\ell_{1}$ regularization term can be expressed as:

$$
\alpha_{n} R\left(x_{\mathbf{p}}\right)=\sum_{p} \alpha_{n, p} \sum_{r}\left|w_{r p}\right|=\sum_{p} \sum_{r} \sqrt{\left|\alpha_{n, p} w_{r p}\right|^{2}}
$$

with $\alpha_{n, p}$ the regularization parameter for the $p$ th parameter map at the $n$th GaussNewton step. Further, because the three parameter maps share the same object, it is very likely that edges in the three maps appear in the same spatial position, which means the wavelet coefficients have a similar distribution. Therefore, a joint sparsity model [120] is employed, i.e.,

$$
\alpha_{n} R\left(x_{\mathbf{p}}\right)=\alpha_{n} \sum_{r} \sqrt{\sum_{p}\left|w_{r p}\right|^{2}} .
$$

By doing so, small coefficients in one map will be protected from being suppressed by nonlinear thresholding if a large coefficient is present in one of the other maps at the same position. In order to apply joint thresholding, all the parameters need to have a similar scaling which is achieved by normalizing each map with respect to its $L^{2}$ norm prior to wavelet transformation.

In addition, as the radial trajectory does not sample the $k$-space corners, a $k$ space filter [27] is added to the sampling pattern $P$ to penalize signals in the undefined corners of $k$-space.

\subsubsection{Optimization}

Combining the regularization terms with equation (7.7), the subproblem we want to minimize in each Gauss-Newton step reads:

$$
x_{n+1}=\underset{x}{\operatorname{argmin}}\left\|D F\left(x_{n}\right)\left(x-x_{n}\right)+F\left(x_{n}\right)-y\right\|_{2}^{2}+\beta_{n} \sum_{j=1}^{N}\left\|\hat{c}_{j}\right\|_{2}^{2}+\alpha_{n} \sum_{r} \sqrt{\sum_{p}\left|w_{r p}\right|^{2}}
$$

where $\hat{c}_{j}$ is the weighted Fourier coefficients of the $j$ th coil [47], i.e., $\hat{c}_{j}=(1+$ $\left.s\|\vec{k}\|^{2}\right)^{l / 2} \mathcal{F} c_{j}$ with $s, l$ scalar constants. The first two terms of the above equa- 
tion are a smooth and convex function of $x$, the $\ell_{1}$ regularization term is a nonsmooth but convex function. Equation (7.10) is solved using the fast iterative shrinkage-thresholding algorithm (FISTA) [121]. Bound constraints for the physical parameters (i.e., $R_{1}^{*}>0$ ) are employed within FISTA. The pseudo-codes of the algorithms are shown in Algorithms 1 and 2 . To apply the above algorithms to the model-based reconstruction the derivative of the operator and the adjoint of the derivative are also needed to be implemented, both of which are provided in the appendix.

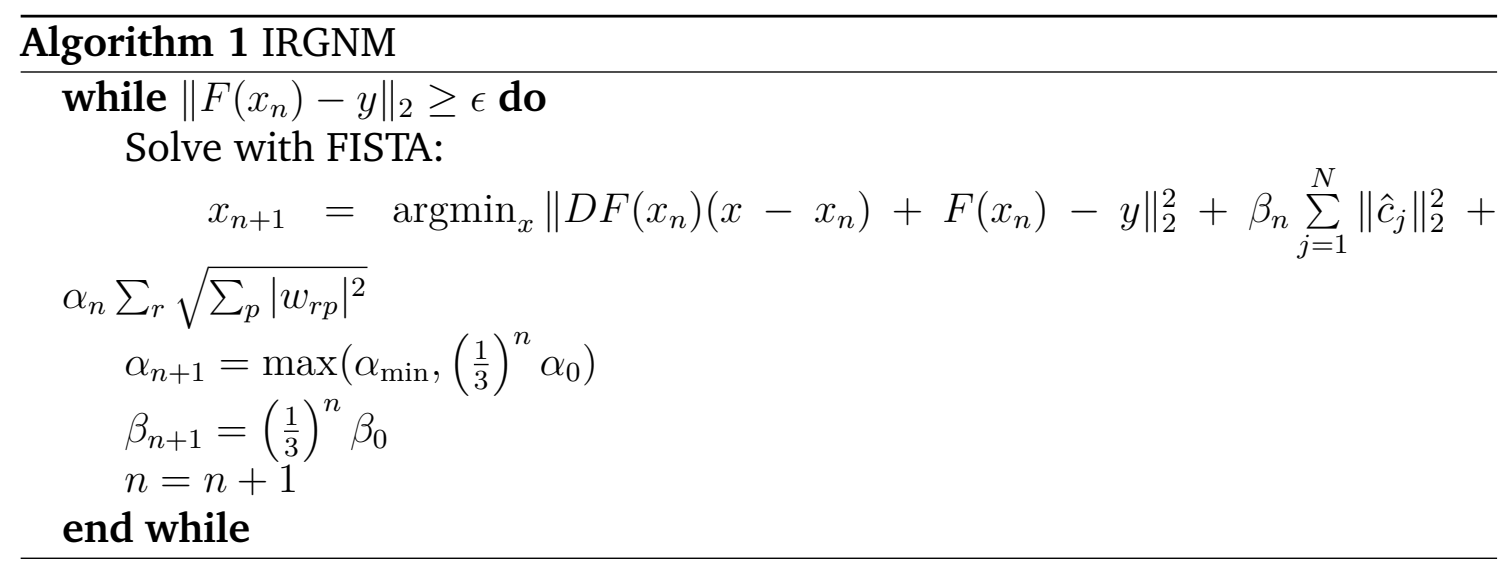

By setting

$$
\begin{aligned}
& g(x)=\left\|D F\left(x_{n}\right)\left(x-x_{n}\right)+F\left(x_{n}\right)-y\right\|_{2}^{2}+\beta_{n} \sum_{j=1}^{N}\left\|\hat{c}_{j}\right\|_{2}^{2} \text { and } \\
& h(x)=\alpha_{n} \sum_{r} \sqrt{\sum_{p}\left|w_{r p}\right|^{2}}
\end{aligned}
$$

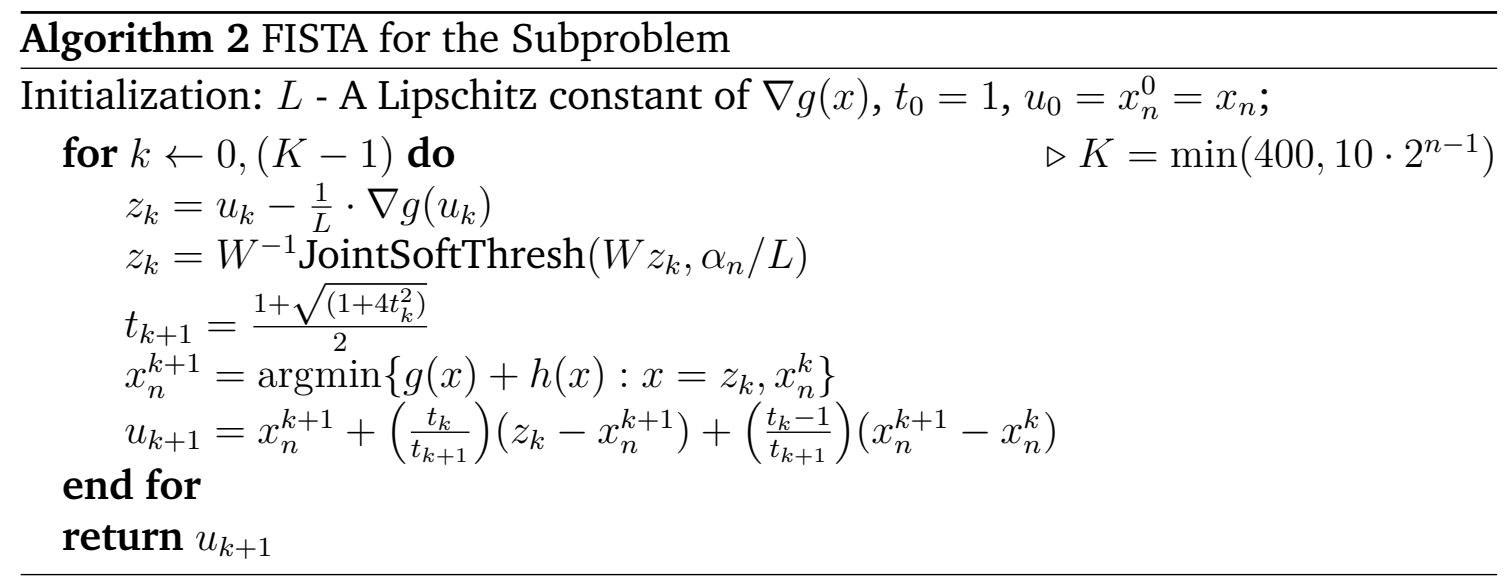




\subsection{Materials and Methods}

\subsubsection{Data Acquisition}

A small golden-angle $\left(\sim 68.75^{\circ}\right)$ sampling scheme is employed here to sample the data continuously during inversion recovery. Spoiling of residual transverse magnetizations was accomplished by random RF phases [78]. Golden-angle based sampling strategy covers the entire $k$-space without repeating any angles and allows for arbitrary binnings of spokes to form one $k$-space frame [73]. Although no intermediate image is needed to be reconstructed using model-based reconstructions, binning spokes from neighborhoods with a proper size helps to reduce the computation time as long as the temporal fidelity is not compromised [64]. Therefore, a small number of spokes (e.g. 21 to 25) is binned together to form one $k$-space frame. The sequence diagram is given in Figure 7.1.

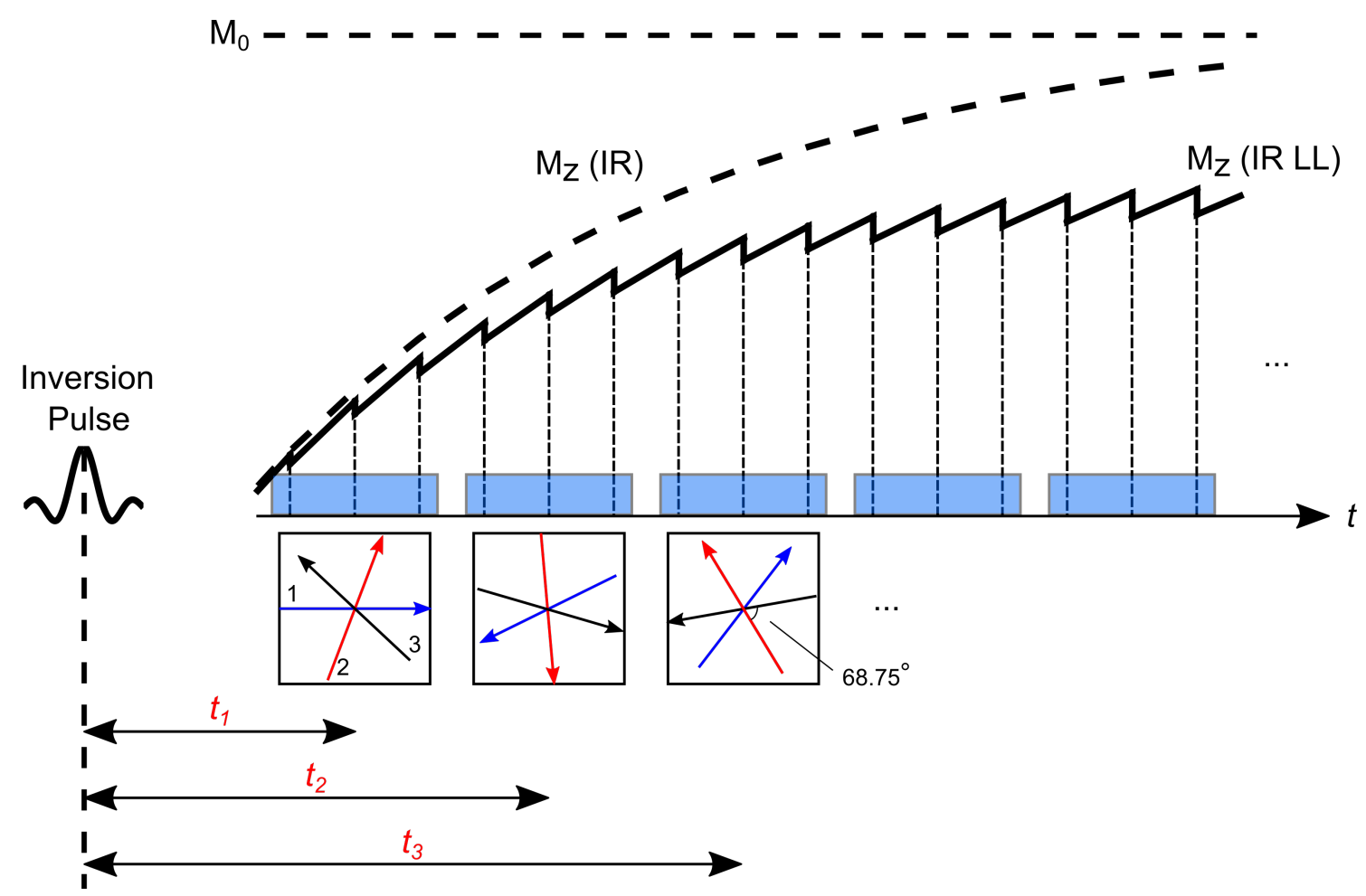

Figure 7.1: Data acquisition scheme of a single-shot IR LL sequence with a small golden-angle readout. $t_{1}, t_{2}, t_{3}$ are the first three inversion times.

\subsubsection{Numerical Simulations}

To evaluate the accuracy of the proposed approach, a numerical phantom composed of three circular objects and one circular background was used. The $k$-space data was derived from the analytical Fourier space representation of an ellipse. Three different $T_{1}$ relaxation times (300 ms, $800 \mathrm{~ms}, 1500 \mathrm{~ms}$ ) with a background 
compartment of $2000 \mathrm{~ms}$ were simulated for the phantom. An array of 4 circular receiver coils was assumed, surrounding the phantom without overlap. The complex sensitivities of these coils were calculated based on Biot-Savart's law and sinusoidal fitting [80]. Complex white Gaussian noise with a standard deviation of 0.1 was added to the simulated $k$-space data. The inversion recovery Look-Locker radial FLASH sequence employing the small golden-angle readout was then used to sample the simulated $k$-space data with a base resolution of 192 pixels covering a FOV of $128 \mathrm{~mm}, \mathrm{TR} / \mathrm{TE}=2.93 / 2.01 \mathrm{~ms}, \mathrm{FA}=4^{\circ}$, total acquisition time $4 \mathrm{~s}$.

\subsubsection{MRI}

All MRI measurements were performed on a human MRI system operating at 3T (Magnetom Prisma fit, Siemens Healthcare, Erlangen, Germany). Phantom and brain studies were conducted with a standard 64-channel head coil, while abdominal scans were performed with an 18-element thorax coil in conjunction with 18 elements of the 32-element spine coil. Subjects without known illness were recruited among the students of the local university and written informed consent, according to the recommendations of the local ethics committee, was obtained from all subjects prior to MRI. The proposed method was validated experimentally with a commercial reference phantom (Diagnostic Sonar LTD, Scotland, UK) consisting of 6 compartments with defined $T_{1}$ values surrounded by water. The sequence protocols for phantom, brain and abdomen studies are summarized in Table 7.1. Abdominal measurements were performed during a brief breath hold.

Fully sampled reference data sets for phantom and brain studies were acquired by combining 21 sequential single-shot IR LL acquired data sets. The radial vieworder for each individual inversion recovery acquisition was varied to yield a uniform distribution of 441 spokes per time point after combination.

\subsubsection{Parameter Choice for Reconstruction}

\section{Scaling}

The convergence speed of the proposed method depends largely on the relative scaling of partial derivatives of the cost function with respect to each component in $x$. The scaling also influences the choice of regularization parameters. In this problem, after normalizing the raw data with respect to the norm of the data acquired at the first inversion time, two additional scalars $L_{M_{0}}$ and $L_{R_{1}^{*}}$ were in- 
Table 7.1: MRI parameters for phantom, human brain and abdomen acquisitions.

\begin{tabular}{lll}
\hline & Phantom/Brain & Abdomen \\
\hline Field-of-view / mm ${ }^{2}$ & $192 \times 192$ & $320 \times 320$ \\
Image matrix size & $256 \times 256$ & $320 \times 320$ \\
Resolution / mm ${ }^{2}$ & $0.75 \times 0.75$ & $1 \times 1$ \\
Slice thickness / mm & 5 & 6 \\
Repetition time (TR) / ms & 2.93 & 2.4 \\
Echo time (TE) / ms & 2.01 & 1.62 \\
Bandwidth / Hz pixel $^{-1}$ & 1030 & 1420 \\
Flip angle / degree & 4 & 4 \\
Spokes per ( $k$-space) frame & 21 & 25 \\
Total acquisition time / s & 4 & 4 \\
\hline
\end{tabular}

troduced to balance the partial derivatives of the three parameters:

$$
\begin{aligned}
M_{0} & =L_{M_{0}} \cdot \hat{M}_{0} \\
R_{1}^{*} & =L_{R_{1}^{*}} \cdot \hat{R}_{1}^{*}
\end{aligned}
$$

with $\hat{M}_{0}$ and $\hat{R}_{1}^{*}$ the parameters actually optimized during reconstruction. $L_{M_{0}}, L_{R_{1}^{*}}$ should be calculated to satisfy $\left\|d \hat{M}_{0}\right\| \approx\left\|d M_{s s}\right\| \approx\left\|d \hat{R}_{1}^{*}\right\|$. Practically, a choice of $L_{M_{0}}=2$ and $L_{R_{1}^{*}}=1$ roughly kept the partial derivatives of the parameters balanced after normalizing the raw data. $L_{M_{0}}$ and $L_{R_{1}^{*}}$ were then set to be 2 and 1 respectively for all the reconstructions in this work.

\section{Initialization}

For Newton-type algorithms, initialization is very important for convergence. In practice this seems to be no problem in the proposed method. With a reasonable choice of $\hat{R}_{1}^{*}$ with $1.5, M_{s s}$ and $\hat{M}_{0}$ with 1 and all coils 0 initially, good convergence were observed in all tested cases.

\section{Regularization Parameters}

Regularization parameters need to be tuned in order to balance the preservation of fine details and the residual noise. In this study, both $\alpha_{0}$ and $\beta_{0}$ were chosen to be 1 and reduced by a factor of 3 (i.e., $q_{\alpha}=q_{\beta}=3$ ) in each Gauss-Newton step. Similar to [119], a minimum value of $\alpha$ was introduced to further control the noise in the later Gauss-Newton steps, i.e., $\alpha_{n+1}=\max \left(\alpha_{\min }, q_{\alpha} \alpha_{n}\right) . \quad \alpha_{\min }$ was tuned from 0.001 to 0.004 (step size 0.001). An optimal value for $\alpha_{\min }$ was determined by experienced radiologists to get good SNR without compromising 
the quantitative accuracy meanwhile. The parameters $s$ and $l$ were chosen to be $440 \mathrm{~mm}^{2}$ and 16 respectively.

\subsubsection{Implementation}

All data processing was done offline. The entire dataset from multiple coils was first corrected for gradient delay errors [52] and then compressed to 10 virtual channels using principle component analysis. A convolution based gridding [53] without density compensation was used to interpolate the radial samples onto a Cartesian grid on which all successive iterative computations were performed. The preprocessing steps were done using MATLAB (MathWorks, Natik, MA) and the main iterative optimization part was implemented in C/CUDA (GeForce GTX TITAN, NVIDIA, Santa Clara, CA). The wavelet transform (Daubechies 4) from the BART toolbox [122] was used for the sparsity regularization. A randomized shifting [123] was employed to reduce the blocky artifacts caused by the lack of translation invariance properties of orthogonal wavelets. The number of inner iterations was initialized with $N_{0}=10$ and increased to $N_{k+1}=\min \left(400,2 N_{k}\right)$ in the $(k+1)$ th Gauss-Newton step. The Lipschitz constant used in FISTA was calculated using the power method with 30 iterations.

\subsection{Results}

\subsubsection{Validation Studies}

Figure 7.2 shows parameter maps $M_{s s}, M_{0}$ and $R_{1}^{*}$ together with the corresponding $T_{1}$ maps for the numerical phantom estimated by model-based reconstruction with $L^{2}$ and $\ell_{1}$ regularizations as well as NLINV plus pixel-wise fitting respectively (rows 1-4). The bottom row shows corresponding line profiles of the $R_{1}$ maps. When compared to the other two methods, the $\ell_{1}$ regularized model-based reconstruction produces parameter maps with a significant reduction of noise. The flat line profile on the $R_{1}$ map reveals that the $T_{1}$ map by $\ell_{1}$ regularized modelbased reconstruction is closest to the true solution. In addition, the model-based reconstruction methods, in accordance with the ground truth, provide a more homogeneous $M_{0}$ map than NLINV (second row in Figure 7.2). A ROI analysis of the $T_{1}$ maps is shown in Table 7.2. Both proposed model-based reconstructions and NLINV have a good $T_{1}$ accuracy when compared to the ground truth. The best precision is achieved by the $\ell_{1}$ regularized model-based reconstruction.

Figure 7.3 shows experimental phantom $T_{1}$ maps from a fully-sampled dataset with a single virtual coil and multiple virtual coils using gridding plus inverse FFT, 
Model-based (L2)

Model-based (L1)

NLINV
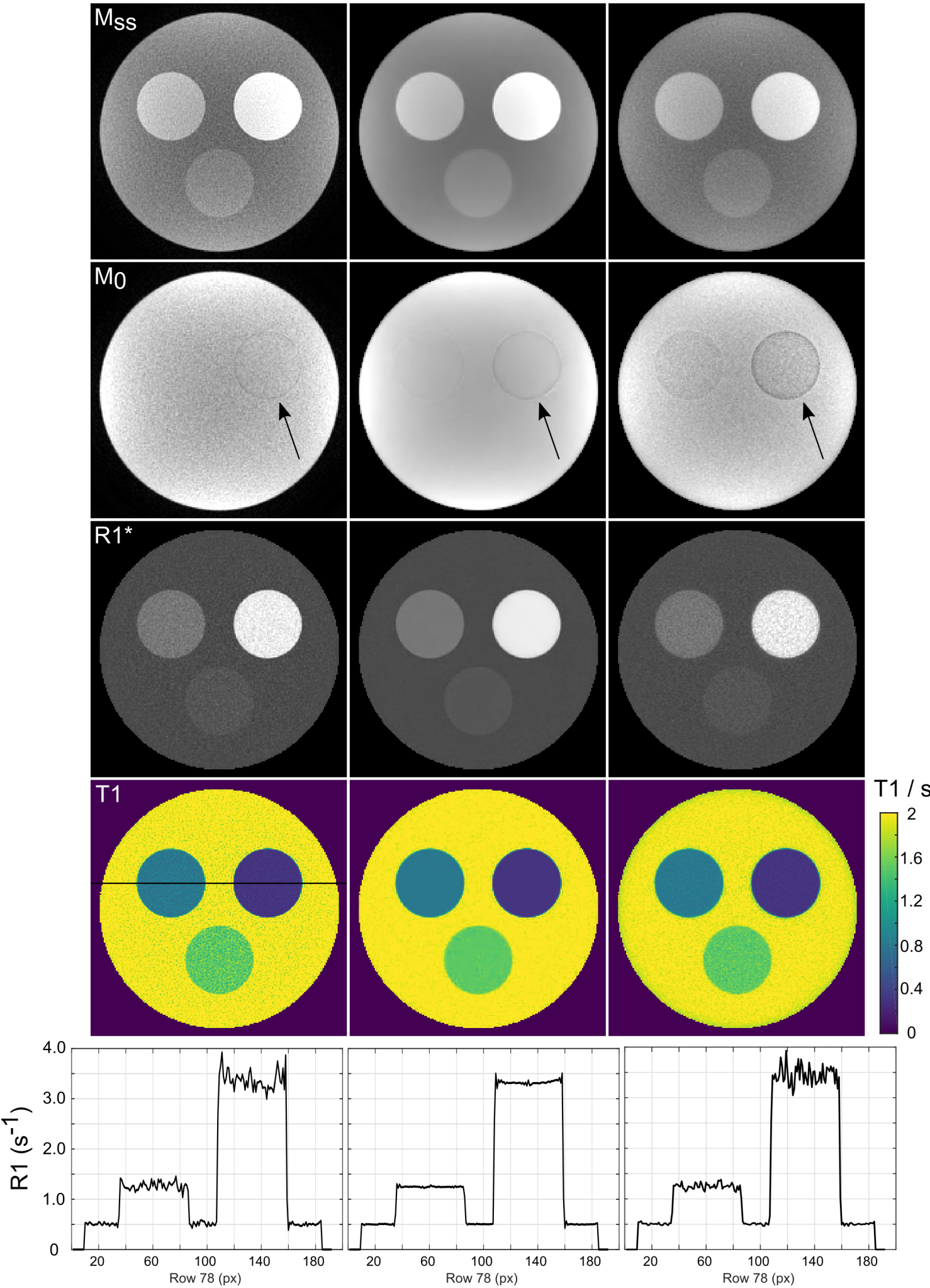

Figure 7.2: Validation of the proposed method in a numerical phantom study. Rows 1-3 show the parameter maps $M_{s s}, M_{0}$, and $R_{1}^{*}$ obtained by model-based reconstruction with $L^{2}, \ell_{1}$ regularization and NLINV reconstruction plus pixel-wise fitting. Row 4 shows the corresponding $T_{1}$ maps. Line profiles of the $R_{1}$ maps are shown in the last Row. Modelbased reconstruction methods provide more homogeneous $M_{0}$ maps than NLINV (arrows). The regularization parameter for the $\ell_{1}$ regularized reconstruction was $\alpha_{\min }=0.003$. 


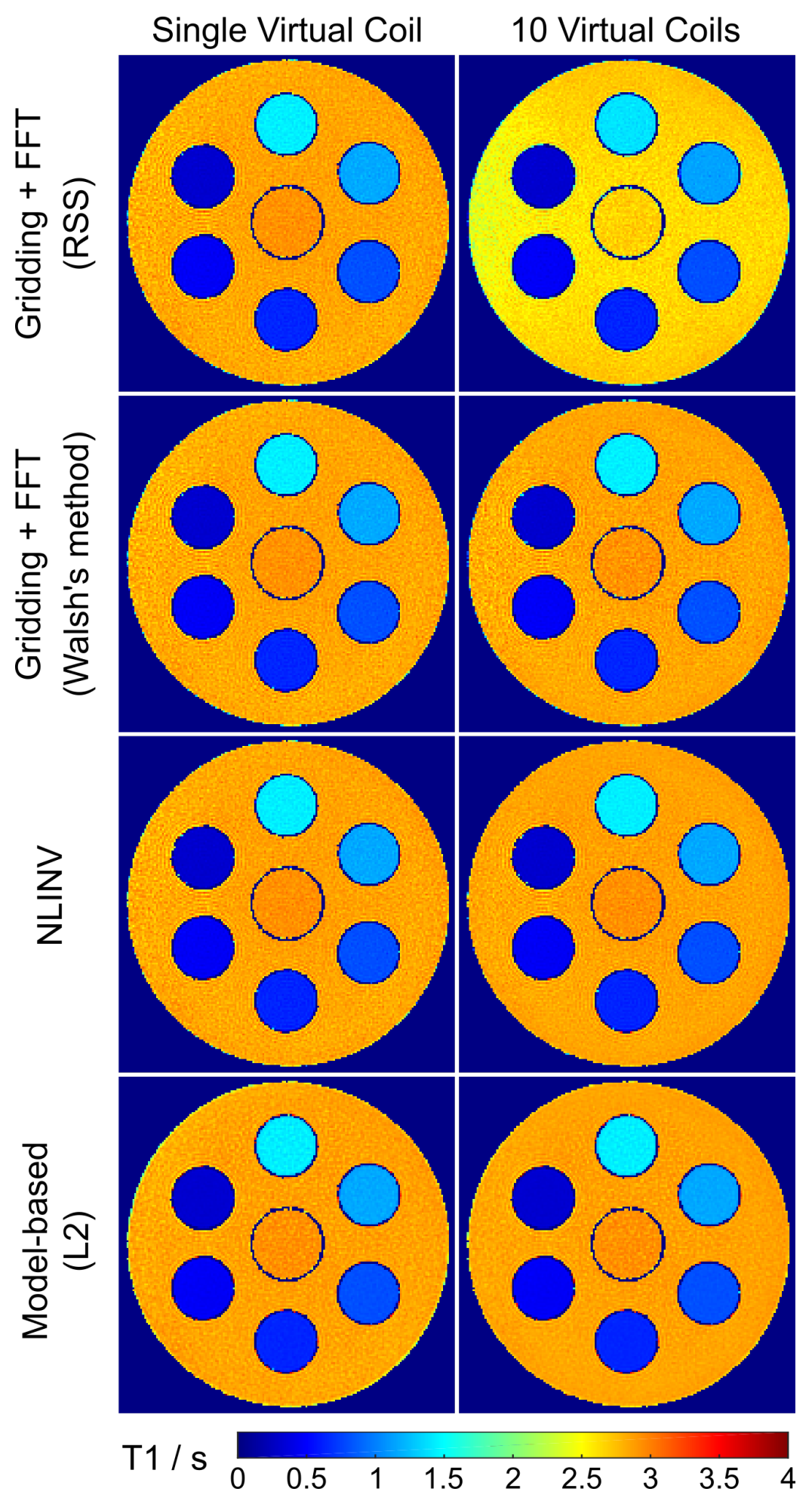

Figure 7.3: Phantom $T_{1}$ maps estimated by different methods from a fully-sampled dataset with a single virtual coil (left) and multiple virtual coils (right). The visible color change in the color-coded $T_{1}$ map of the first row indicates a bias caused by the RSS operation. The other three methods do not have this problem. 
Fully-sampled

Model-based (L2)
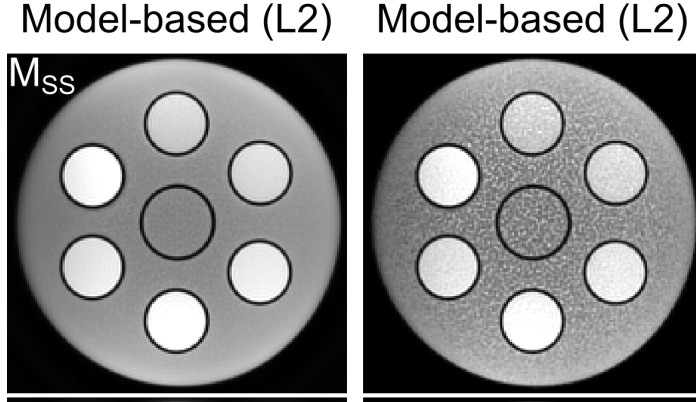

Single-shot
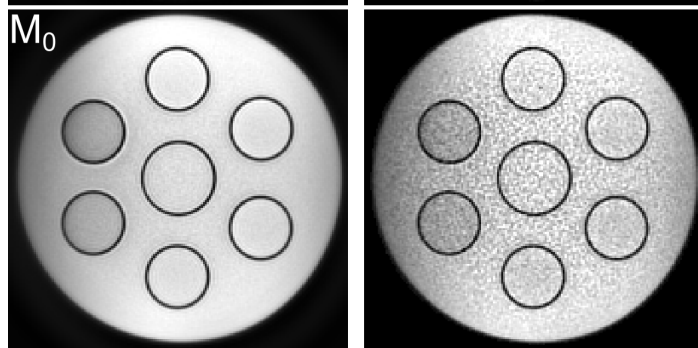

Model-based (L1)

NLINV
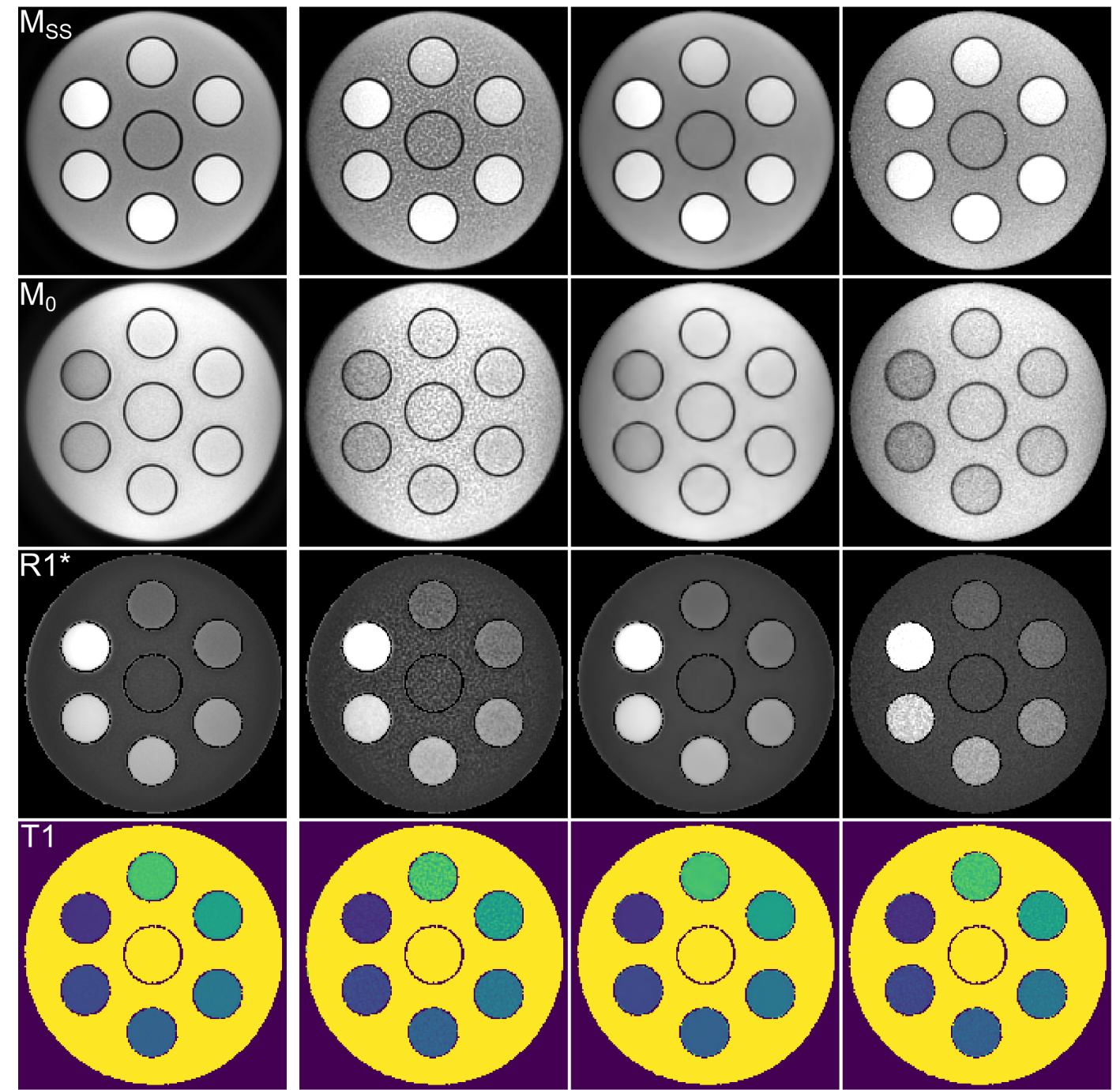

$\mathrm{T} 1 / \mathrm{s}$
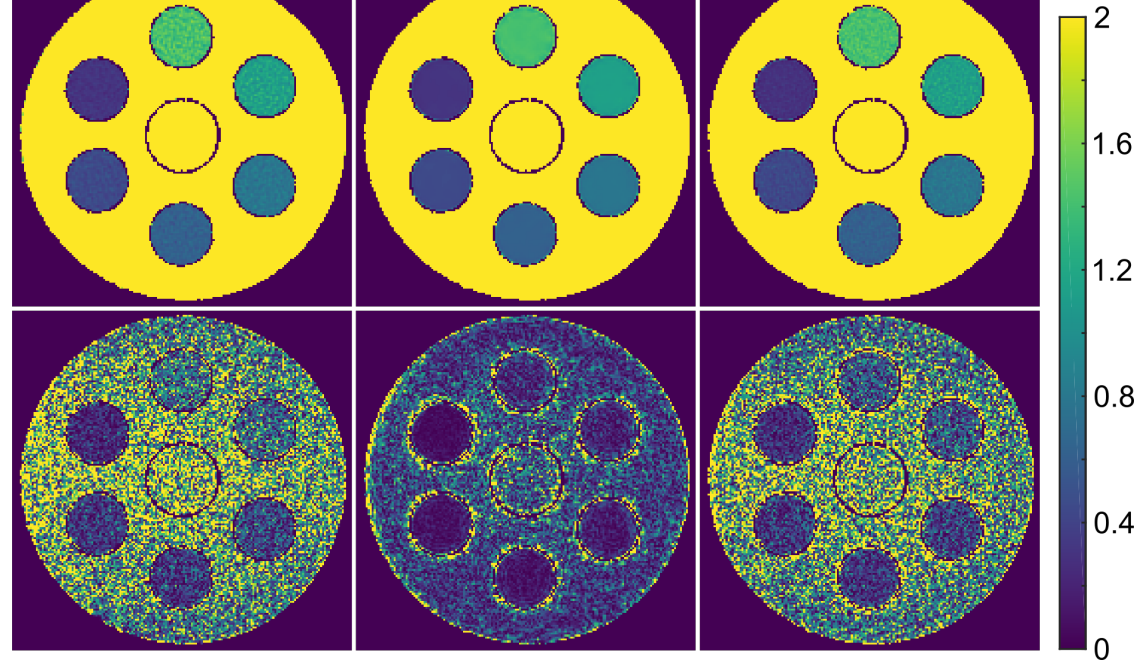

Figure 7.4: Validation of the proposed method in an experimental phantom study. (Left) Parameter maps $M_{s s}, M_{0}, R_{1}^{*}$ and $T_{1}$ from a fully sampled data set by $L^{2}$ regularized model-based reconstruction. (Second column to right) Parameter maps from a single-shot IR LL sampled data by $L^{2}, \ell_{1}$ regularized model-based reconstruction and NLINV respectively. The corresponding $T_{1}$ differences $(\times 10)$ to the fully-sampled case are shown in the fifth row. The regularization parameter for the $\ell_{1}$ regularized reconstruction was $\alpha_{\min }=0.003$. For measurement details see Table 7.1 . 
Table 7.2: Quantitative evaluations of the $T_{1}$ maps (ms) for the numerical phantom shown in Figure 7.2 .

\begin{tabular}{ccccr}
\hline & $\begin{array}{c}\text { True } \\
T_{1}\end{array}$ & $\begin{array}{c}\text { Model-based } \\
\left(L^{2}\right)\end{array}$ & $\begin{array}{c}\text { Model-based } \\
\left(\ell_{1}\right)\end{array}$ & NLINV \\
\hline Tube 1 & 300 & $300 \pm 19$ & $301 \pm 6$ & $300 \pm 18$ \\
Tube 2 & 800 & $802 \pm 47$ & $800 \pm 6$ & $804 \pm 35$ \\
Tube 3 & 1500 & $1500 \pm 77$ & $1498 \pm 17$ & $1498 \pm 58$ \\
Background & 2000 & $1997 \pm 101$ & $2000 \pm 25$ & $1989 \pm 74$ \\
\hline
\end{tabular}

Results represent mean values \pm standard deviations.

Table 7.3: Quantitative evaluations of the experimental phantom $T_{1}$ maps (Values given in $\mathrm{ms}$ ).

\begin{tabular}{cccccccc}
\hline Tube & $\begin{array}{c}\text { Fully- }^{1} \\
\text { sampled }\end{array}$ & $\begin{array}{c}\text { Model-based } \\
\left(L^{2}\right)\end{array}$ & $\begin{array}{c}\text { diff } \\
(\%)\end{array}$ & $\begin{array}{c}\text { Model-based } \\
\left(\ell_{1}\right)\end{array}$ & $\begin{array}{c}\text { diff } \\
(\%)\end{array}$ & NLINV & $\begin{array}{r}\text { diff } \\
(\%)\end{array}$ \\
\hline 1 & $311 \pm 8$ & $309 \pm 30$ & 0.6 & $309 \pm 9$ & 0.6 & $295 \pm 27$ & 5.1 \\
2 & $458 \pm 12$ & $454 \pm 47$ & 0.9 & $451 \pm 12$ & 1.5 & $445 \pm 39$ & 2.8 \\
3 & $633 \pm 10$ & $629 \pm 45$ & 0.6 & $625 \pm 15$ & 1.3 & $621 \pm 35$ & 1.9 \\
4 & $805 \pm 14$ & $798 \pm 56$ & 0.9 & $793 \pm 20$ & 1.5 & $791 \pm 43$ & 1.7 \\
5 & $1158 \pm 21$ & $1152 \pm 81$ & 0.5 & $1146 \pm 45$ & 1.0 & $1146 \pm 64$ & 1.0 \\
6 & $1441 \pm 37$ & $1432 \pm 80$ & 0.6 & $1430 \pm 46$ & 0.8 & $1428 \pm 63$ & 0.9 \\
\hline
\end{tabular}

${ }^{1}$ Fully-sampled $T_{1}$ map is obtained by $L^{2}$ regularized model-based reconstruction.

${ }^{2}$ Given is the relative difference of mean values to the fully sampled data.

NLINV and $L^{2}$ regularized model-based reconstruction, respectively. Images from different coils after gridding are combined using Root of Sum of Squares (RSS) and a matched filter based technique [40] prior to pixel-wise fitting. The visible color change in the color-coded $T_{1}$ maps from single coil to multiple coils in the first row indicates a bias caused by the RSS operation. The other three methods do not have this problem. Results from the $\ell_{1}$ regularized model-based reconstruction are not presented as it is not necessary to apply $\ell_{1}$ regularization in the reconstruction of fully-sampled data.

Figure 7.4 shows a comparison of different methods for an experimental phantom study. Reference is taken from the $L^{2}$ regularized model-based reconstruction of the fully sampled dataset. Noise increases significantly on the parameter maps when the data set is reduced from fully-sampled to single-shot (undersampling factor 21) in $L^{2}$ regularized model-based reconstruction as well as in the NLINV based method. This is largely overcome by the $\ell_{1}$ regularized model-based reconstruction. Consequently, the corresponding $T_{1}$ map of the $\ell_{1}$ regularized modelbased reconstruction has a smallest residual error with respect to the reference. The quantitative ROI analyses of the $T_{1}$ maps in Table 7.3 confirm the accuracy of 
the proposed model-based reconstructions and NLINV. In general, model-based reconstructions have a better accuracy (maximum relative difference rate 1.5\%, $0.9 \%$ for $\ell_{1}$ and $L^{2}$ respectively) than NLINV (maximum relative difference rate $5.1 \%$ ). For all tubes the standard deviations in the $T_{1}$ maps produced by $\ell_{1}$ regularized model-based reconstruction are much smaller than the other two methods.

\subsubsection{Human Studies}

\section{Comparison of Different Regularization Parameters}

Figure 7.5 shows in vivo $T_{1}$ maps by model-based reconstruction with $\ell_{1}$ regularization for four representative sparsity regularization parameters. Low values of $\alpha_{\min }$ result in noisy $T_{1}$ maps while high values introduce blurring. A $\alpha_{\min }$ of 0.003 in brain and 0.002 in liver provide a good trade-off between noise reduction and detail preservation.

\section{Brain and Abdomen Studies}

Figure 7.6 compares brain parameter maps together with $T_{1}$ maps by different methods. The reference is again estimated from the fully sampled dataset by $L^{2}$ regularized model-based reconstruction. In line with the results for the experimental phantom study, noise in the $T_{1}$ maps reconstructed by $L^{2}$ regularized modelbased reconstruction as well as by NLINV is much higher than in the fully sampled data set. The suppression of noise is again most successful in the $\ell_{1}$ regularized model-based reconstruction. Estimated $T_{1}$ map by $\ell_{1}$ regularized model-based reconstruction is also closest to the reference. Comparing to the fully-sampled case, the $\ell_{1}$ regularized model-based reconstructed maps suffer from blurring and reduced image contrast. This is mainly due to the high undersampling factor which is similar to compressed sensing artifact and is not because of the reconstruction parameter selection (as seen in the Figure 7.5). In addition, parameter maps estimated by NLINV are more blurry than those by the model-based reconstructions. One possible reason may be that the images reconstructed by NLINV at such a high undersampling factor are normally noisy and not artifact free, a pixel-wise fitting will reduce the noise and artifacts but at a cost of blurring the final results.

Figure 7.7 shows estimated coil sensitivities after PCA by NLINV and $\ell_{1}$ regularized model-based reconstruction, respectively. No significant visual difference is observed, indicating that the proposed method estimates coil sensitivities well.

Figure 7.8 shows a comparison of the three parameters $M_{s s}, M_{0}$ and $R_{1}^{*}$ together with the $T_{1}$ maps by model-based reconstructions and NLINV in the abdominal study. Again, compared to the other two methods, both noise reduction and spatial 

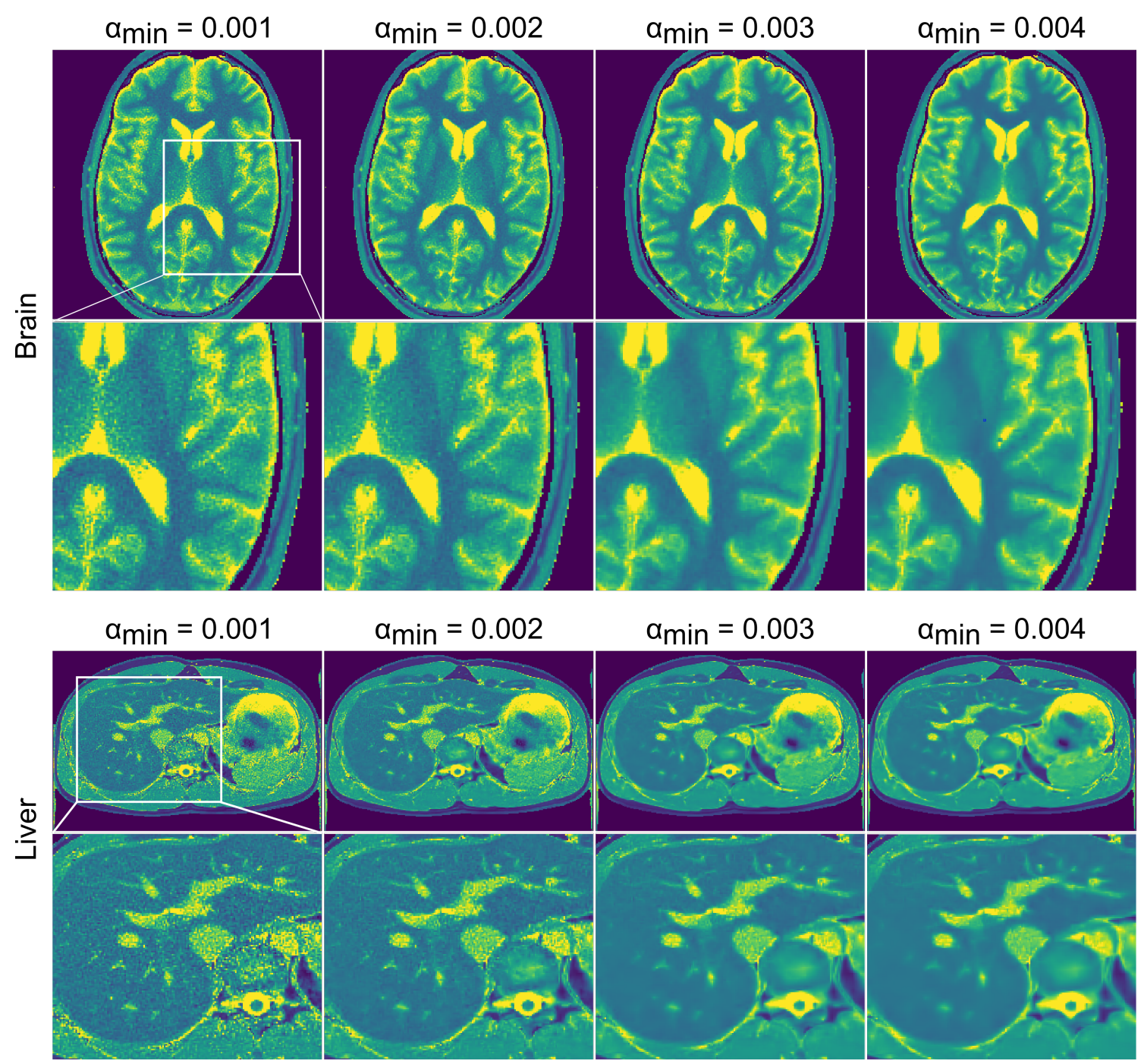

$\mathrm{T} 1 / \mathrm{s}$

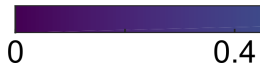

0.8

1.2

1.6

Figure 7.5: Model-based reconstructed $T_{1}$ maps with sparsity constraints using four representative regularization parameters $\left(\alpha_{\min }\right)$ in brain and abdomen studies. Low values of $\alpha_{\text {min }}$ resulted in noisy $T_{1}$ maps while high values introduced blurring. A $\alpha_{\min }$ of 0.003 in brain and 0.002 in liver provided a good trade-off between noise reduction and detail preservation. 
Fully-sampled

Single-shot
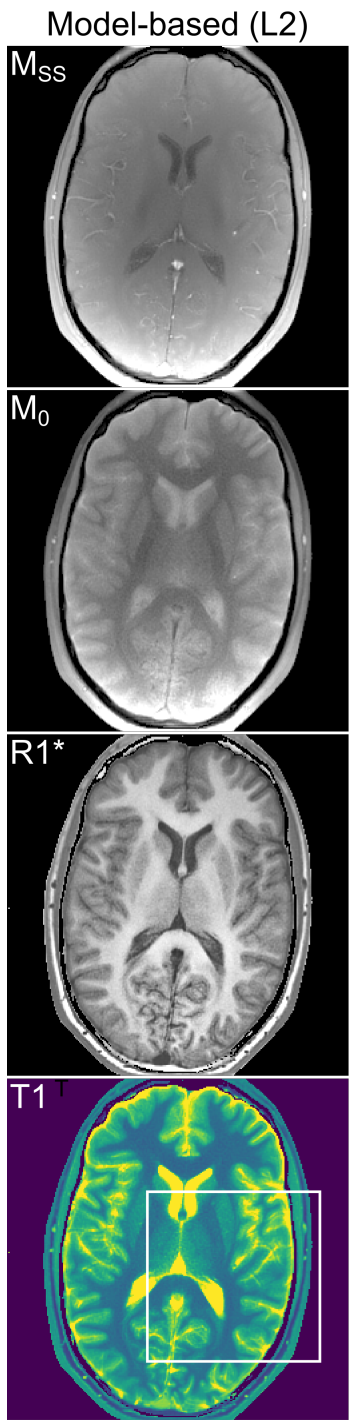

Model-based (L2)

Model-based (L1)

\section{NLINV}
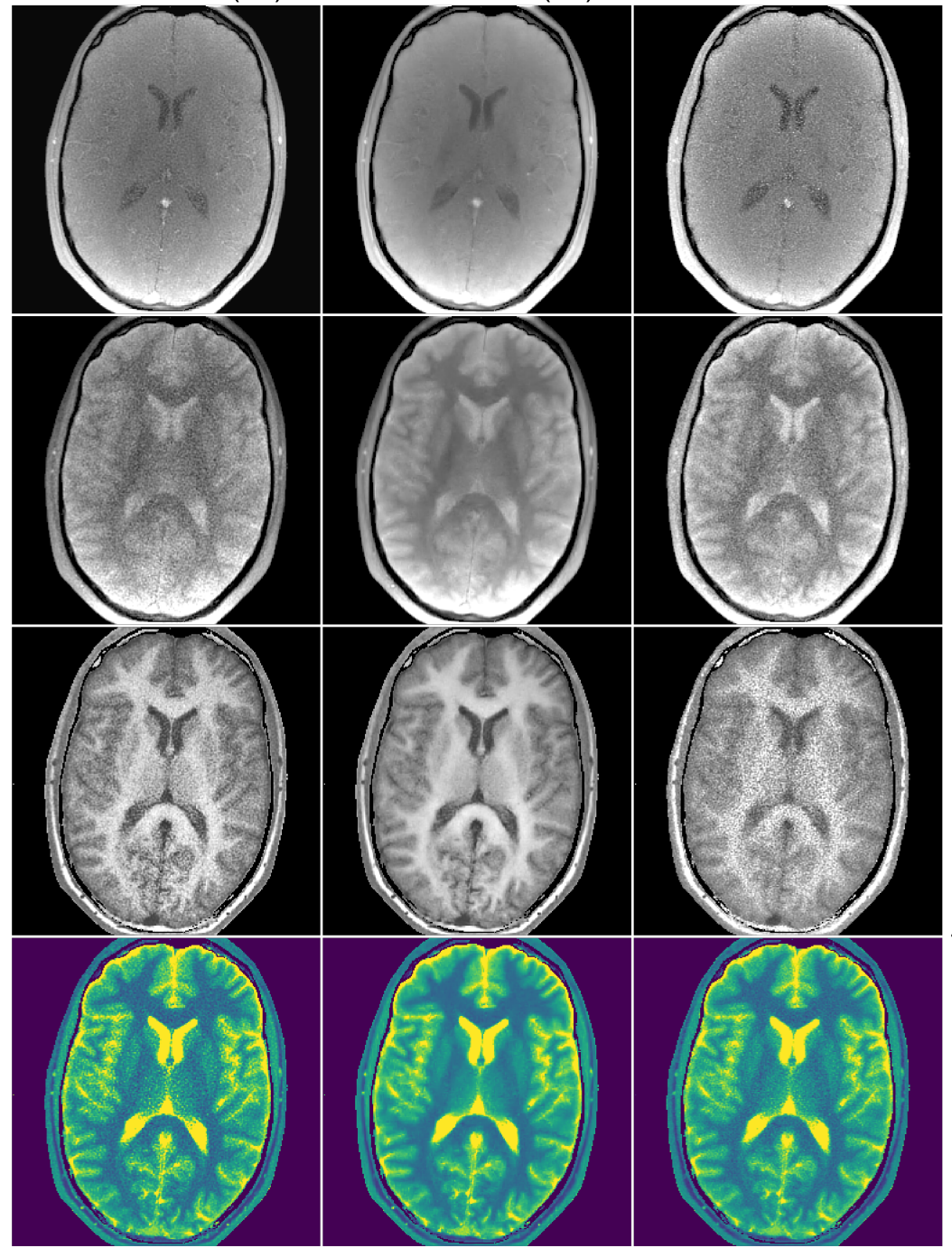

1.2
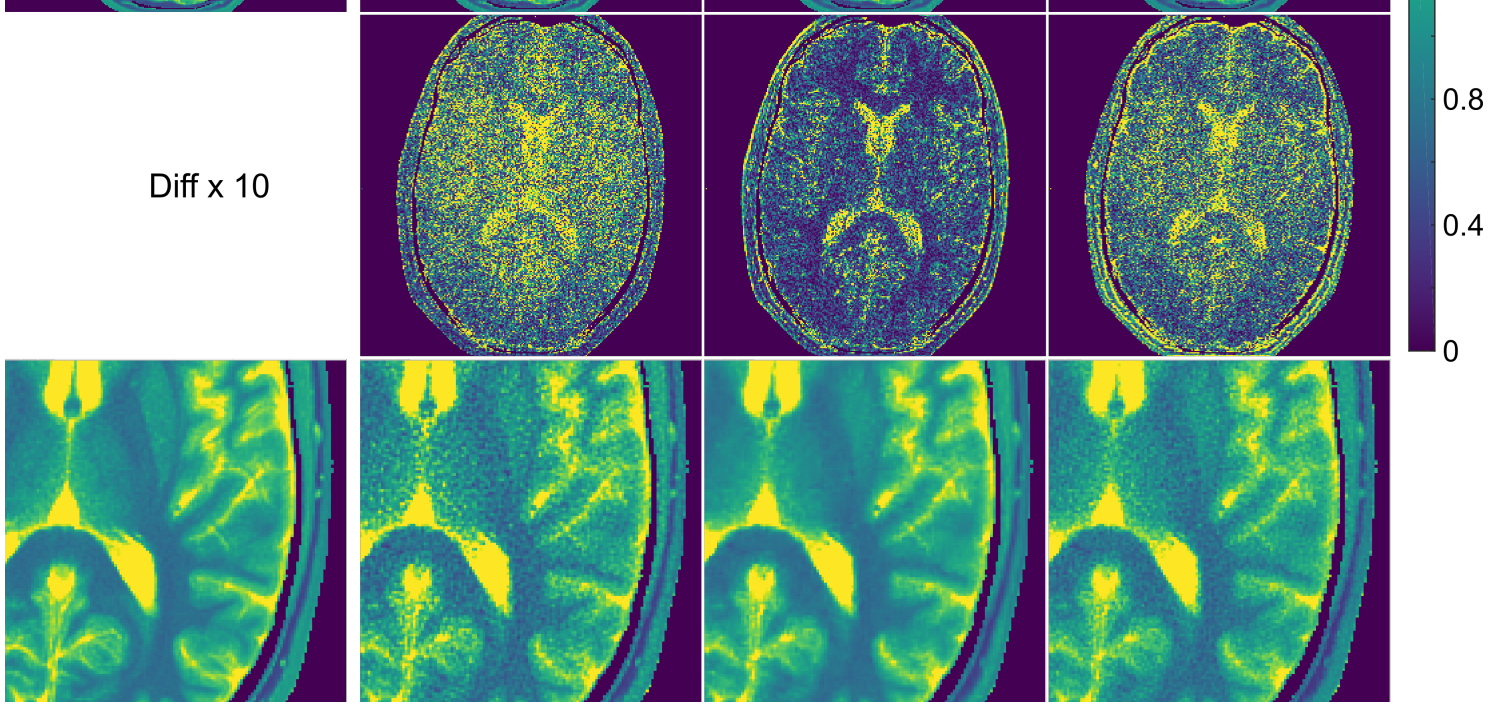

Figure 7.6: (Left, rows 1-4) Brain parameter maps $M_{s s}, M_{0}, R_{1}^{*}$ and $T_{1}$ maps from a fully sampled data by $L^{2}$ regularized model-based reconstruction. (Second column to right, rows 1-4) Parameter maps from a single-shot IR LL sampled data by $L^{2}, \ell_{1}$ regularized model-based reconstruction and NLINV respectively. The corresponding $T_{1}$ differences $(\times 10)$ to the fully-sampled case are shown in the fifth row. The sixth row shows a magnified view of the $T_{1}$ maps. For measurement details see Table 7.1. 


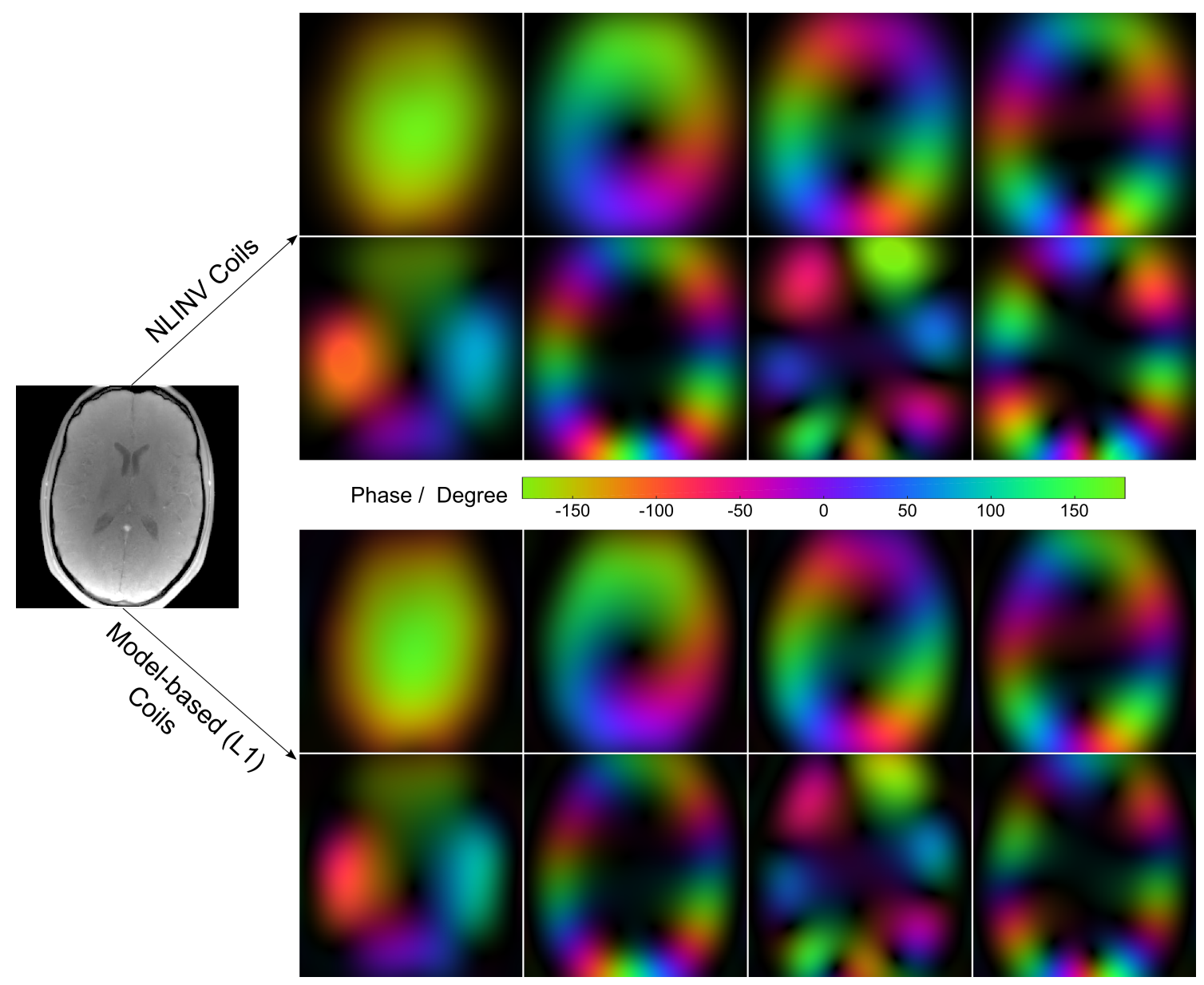

Figure 7.7: Estimated complex coil sensitivities (after PCA) by NLINV (top) and sparsity constrained model-based reconstruction (bottom) for a brain study. 
definition of image details have been significantly improved by the $\ell_{1}$ regularized model-based reconstruction. Noteworthy, in contrast to brain studies, a comparison to a fully-sampled dataset is infeasible as the data acquisition takes more than 7 minutes which precludes a single breath-hold.

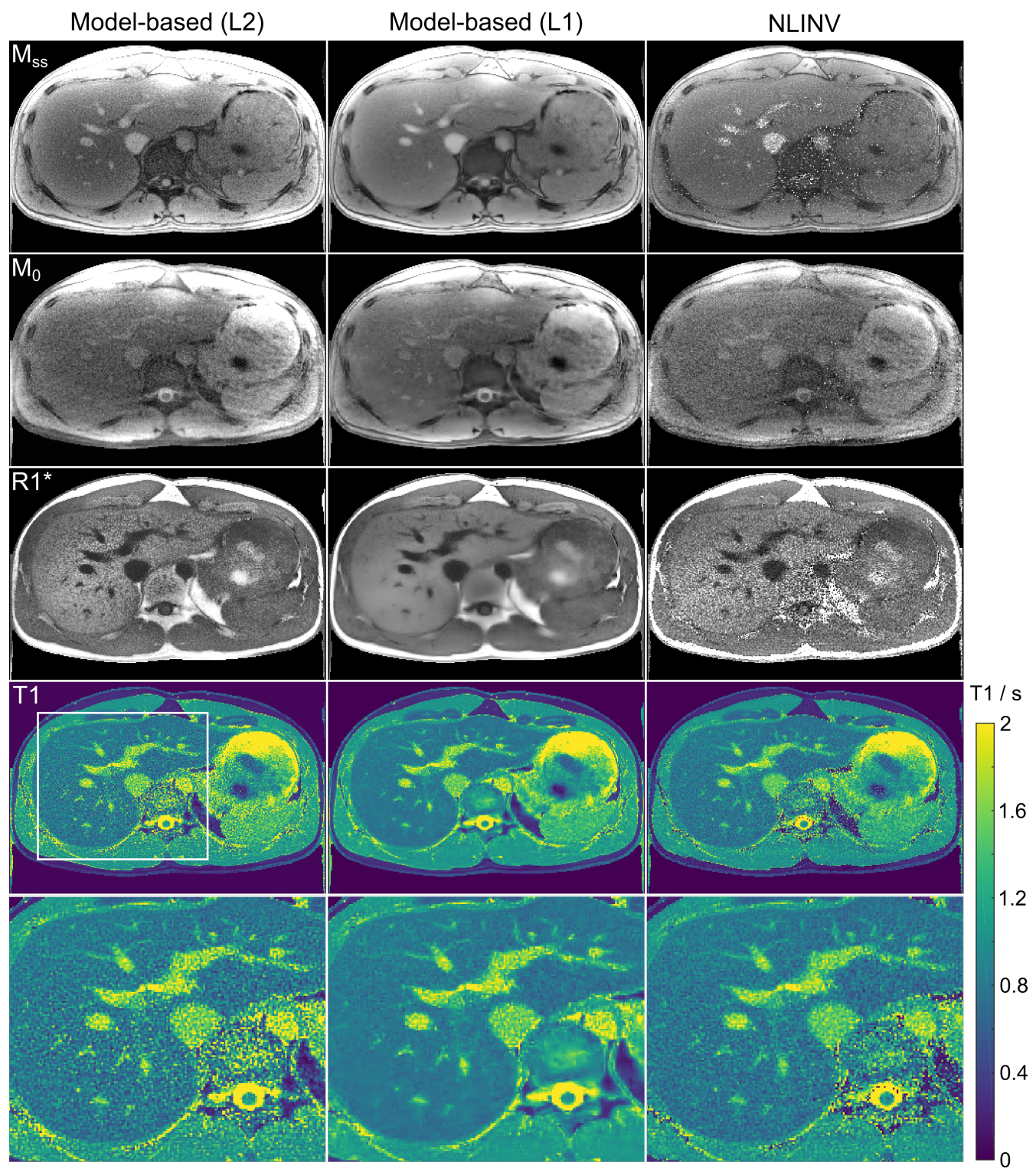

Figure 7.8: Abdomen parameter maps and $T_{1}$ maps estimated by model-based reconstructions and NLINV from single-shot IR LL sampled data with a brief breath-hold. For measurement details see Table 7.1 .

\section{Comparison to NLINV with Denoising}

For NLINV based methods, a recently proposed modified non-local means filter [54] can be used as a post-processing step on the NLINV reconstructed images prior to pixel-wise fitting. It helps to improve the precision of the resulting $T_{1}$ maps. Figure 7.9 (a) shows a comparison of brain $T_{1}$ maps by $\ell_{1}$ regularized 
(a)

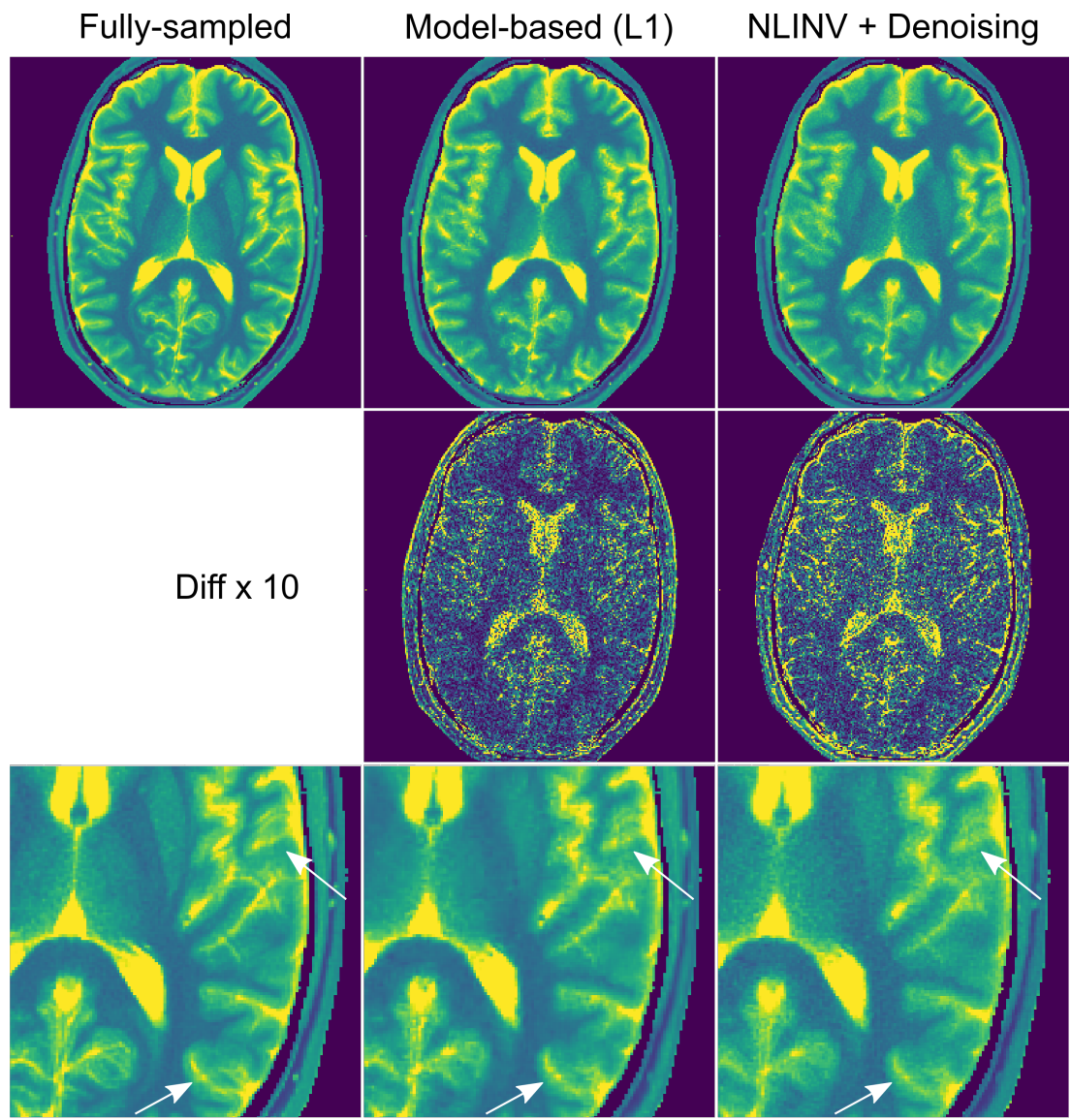

Model-based (L1)

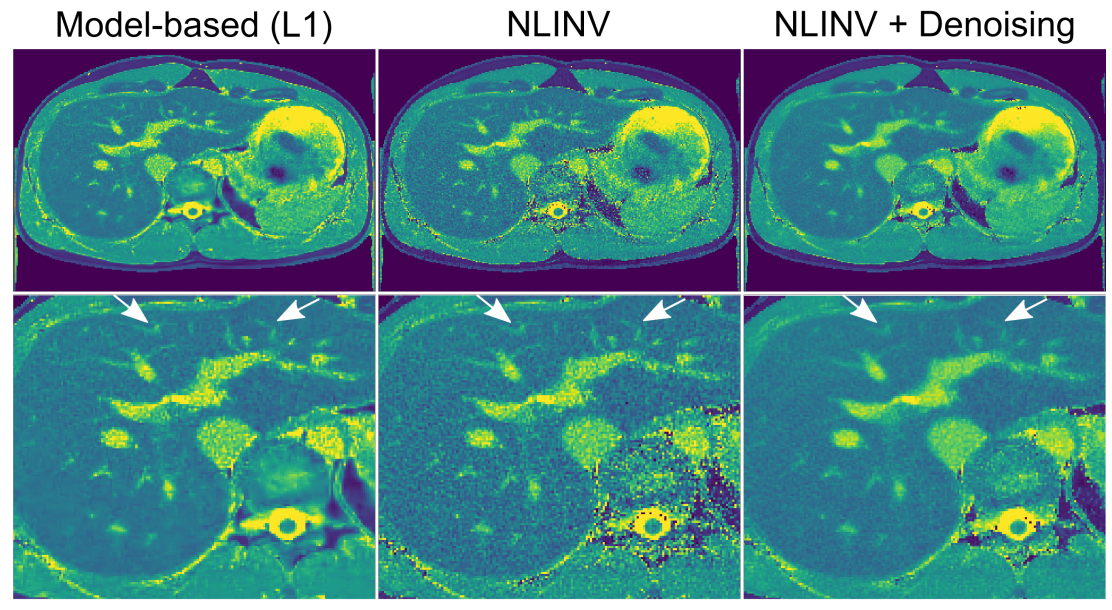

NLINV + Denoising

(b)

$\mathrm{T} 1 / \mathrm{s}$

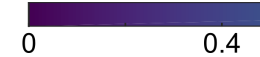

0.8

1.2

1.6

Figure 7.9: Comparison of brain and abdomen $T_{1}$ maps estimated by $\ell_{1}$ regularized model-based reconstruction and NLINV with a denoising filter applied to the reconstructed images prior to pixel-wise fitting. The fullysampled brain $T_{1}$ map and the abdominal $T_{1}$ map by NLINV are presented for comparisons. White arrows indicate blurring effects caused by the denoising filter. 
model-based reconstruction and NLINV with denoising to the reference. Figure 7.9 (b) compares $T_{1}$ maps of abdomen by $\ell_{1}$ regularized model-based reconstruction, NLINV and NLINV with denoising respectively. The denoising filter applied to the images before fitting helps to remove noise in the corresponding $T_{1}$ maps while it also introduces blurring effects especially in the edge regions (e.g., blood vessel borders) indicated by the white arrows. In contrast, the $\ell_{1}$ regularized modelbased reconstruction has a better balance between noise removal and preservation of details.

\subsection{Discussion}

This work presents a model-based reconstruction method which jointly estimates parameter maps and coil sensitivities from single-shot IR Look-Locker radially acquired data. A Wavelet-based $\ell_{1}$ regularization is applied on the parameter maps to improve the performance. Accuracy of the proposed method is confirmed on results from simulation, experimental phantom and brain studies. A pronounced improvement of precision by the sparsity constraints have been observed in all studies. The proposed method allows for a fast high resolution $T_{1}$ mapping within 4 seconds.

Comparing to an existing single-shot Look-Locker $T_{1}$ mapping method based on NLINV [81], the proposed model-based reconstruction bypasses the intermediate image reconstruction step and therefore allows for an arbitrary choice of spoke binning and does not need to compromise between temporal resolution and image quality. Further, model-based reconstruction allows for a direct application of regularization on the parameter maps during reconstruction, which is important for high acceleration factors. The $\ell_{1}$ regularized model-based reconstruction produced $T_{1}$ maps with a much higher precision, more spatial defined information and is closer to the map estimated from the fully-sampled dataset than NLINV. In comparison to the method by NLINV plus denoising before pixel-wise fitting, the $\ell_{1}$ regularized model-based reconstruction can achieve a better balance between noise reduction and detail preservation. In addition, phantom results demonstrates a slightly increased accuracy of the proposed model-based reconstructions than the NLINV based method.

Another model-based approach (MAP) [76, 87] has been proposed for singleshot Look-Locker parameter mapping at a relatively low resolution $(1.6 \times 1.6 \times$ $4 \mathrm{~mm}^{3}$ ). The MAP method iteratively fits the parameters of the mono-exponential model to the undersampled radial $k$-space data. In contrast, the proposed method formulates the estimation of unknowns as a nonlinear inverse problem, which offers a greater flexibility for incorporation of additional nonlinear constraints and 
regularization terms. Such options are very important for high acceleration factors necessary in fast high resolution $T_{1}$ mapping. Another critical point for MAP is that it uses the RSS operation to combine different coil images during its iteration. The RSS combination assumes that the coil sensitivities can be approximated by the image itself in the regions of high signal. For inversion recovery based imaging, images around the null point usually have a very low signal intensity, which violates the assumption. Therefore, the combined image will be biased, which will in consequence bias the final $T_{1}$ map. This phenomenon has been observed even for a fully-sampled dataset as shown in Figure 7.3. This operation is not needed for the proposed method.

Another potential advantage of the proposed method is the joint estimation of parameter maps and coil sensitivities from the whole data. When combined with parallel imaging, most model-based reconstructions rely on the coil information pre-estimated by methods such as NLINV or ESPIRiT [31] from a subset of data. The separation of coil calibration and parameter estimation reduces the complexity of the whole problem. However, calibration of coil sensitivities from a subset of data is suboptimal and any inaccuracy in the first step will bring errors to the later step of parameter estimation. The proposed method on the other hand reconstructs parameter maps and coil sensitivities simultaneously using all available data. Similar to autocalibrated parallel imaging method [46, 47], this could potentially improve the calibration of coil sensitivities and in consequence improve the performance of model-based reconstructions.

At this moment, the most relevant limitation of the proposed method is the need for a time consuming offline calculation. Comparing to image-space based methods such as NLINV, model-based reconstructions occupy more memory because all data acquired during inversion recovery is needed in one operation during iteration and therefore are computationally more expensive. Although the main optimization part has been transformed into a C/CUDA implementation, part of the implementation still relies on the CPU version (e.g., wavelet transform), the communication between CPU and GPU then largely prolongs the whole computation time. So far it takes 10 to 20 minutes depending on the matrix size for reconstruction of a dataset used in this work. Further optimization of the implementation could yield an acceleration factor of at least $2-5$.

The proposed method might proof as a useful tool for high resolution myocardial $T_{1}$ mapping. Its combination of very short acquisition times, which are critically required in myocardial parameter mapping, with good accuracy and precision makes the proposed method an attractive alternative to existing methods [91]. 


\subsection{Summary}

In summary, a model-based reconstruction method which jointly estimates parameter maps and coil sensitivities is proposed to accelerate high resolution $T_{1}$ mapping using single-shot IR Look-Locker radial $k$-space data. Sparsity regularization serves as a stabilization term against noise amplification, which otherwise limits the acceleration factor for high resolution $T_{1}$ mapping. With this method, a high-resolution $T_{1}$ map can be generated from data acquired within 4 seconds. Compared to other existing methods, the proposed method is more accurate and has a better precision.

\subsection{Appendix}

\subsubsection{Derivative and adjoint derivatives of the operators}

Due to the linearity of the Fourier transformation and the product rule of derivatives, the derivate of the operator can be calculated as

$$
D F(x)\left(\begin{array}{c}
d M_{s s} \\
d M_{0} \\
d R_{1}^{*} \\
d c_{1} \\
\vdots \\
d c_{N}
\end{array}\right)=\left(\begin{array}{c}
P_{1} \mathcal{F}\left\{c _ { 1 } \left(\frac{\partial M\left(t_{1}\right)}{\partial M_{s s}} d M_{s s}+\frac{\partial M\left(t_{1}\right)}{\partial M_{0}} d M_{0}\right.\right. \\
\left.\left.+\frac{\partial M\left(t_{1}\right)}{\partial R_{1}^{*}} d R_{1}^{*}\right)+M\left(t_{1}\right) d c_{1}\right\} \\
\vdots \\
P_{1} \mathcal{F}\left\{c _ { N } \left(\frac{\partial M\left(t_{1}\right)}{\partial M_{s s}} d M_{s s}+\frac{\partial M\left(t_{1}\right)}{\partial M_{0}} d M_{0}\right.\right. \\
\left.\left.+\frac{\partial M\left(t_{1}\right)}{\partial R_{1}^{*}} d R_{1}^{*}\right)+M\left(t_{1}\right) d c_{N}\right\} \\
\vdots \\
P_{n} \mathcal{F}\left\{c _ { N } \left(\frac{\partial M\left(t_{n}\right)}{\partial M_{s s}} d M_{s s}+\frac{\partial M\left(t_{n}\right)}{\partial M_{0}} d M_{0}\right.\right. \\
\left.\left.+\frac{\partial M\left(t_{n}\right)}{\partial R_{1}^{*}} d R_{1}^{*}\right)+M\left(t_{n}\right) d c_{N}\right\}
\end{array}\right)
$$

and the adjoint can then be derived as

$$
\left(\begin{array}{c}
d M_{s s} \\
d M_{0} \\
d R_{1}^{*} \\
d c_{1} \\
\vdots \\
d c_{N}
\end{array}\right)=D F^{H}(x)\left(\begin{array}{c}
y_{1,1} \\
y_{2,1} \\
\vdots \\
y_{n, N}
\end{array}\right)=\left(\begin{array}{c}
\sum_{j=1}^{N} \sum_{k=1}^{n}\left(\frac{\partial M\left(t_{k}\right)}{\partial M_{s s}}\right)^{*} \cdot c_{j}^{*} \cdot \mathcal{F}^{-1}\left\{P_{k} y_{k, j}\right\} \\
\sum_{j=1}^{N} \sum_{k=1}^{n}\left(\frac{\partial M\left(t_{k}\right)}{\partial M_{0}}\right)^{*} \cdot c_{j}^{*} \cdot \mathcal{F}^{-1}\left\{P_{k} y_{k, j}\right\} \\
\sum_{j=1}^{N} \sum_{k=1}^{n}\left(\frac{\partial M\left(t_{k}\right)}{\partial R_{1}^{*}}\right)^{*} \cdot c_{j}^{*} \cdot \mathcal{F}^{-1}\left\{P_{k} y_{k, j}\right\} \\
\sum_{k=1}^{n} M\left(t_{k}\right)^{*} \cdot \mathcal{F}^{-1}\left\{P_{k} y_{k, 1}\right\} \\
\vdots \\
\sum_{k=1}^{n} M\left(t_{k}\right)^{*} \cdot \mathcal{F}^{-1}\left\{P_{k} y_{k, N}\right\}
\end{array}\right)
$$


with partial derivatives

$$
\begin{aligned}
& \frac{\partial M\left(t_{k}\right)}{\partial M_{s s}}=1-e^{-R_{1}^{*} \cdot t_{k}} \\
& \frac{\partial M\left(t_{k}\right)}{\partial M_{0}}=e^{-R_{1}^{*} \cdot t_{k}} \\
& \frac{\partial M\left(t_{k}\right)}{\partial R_{1}^{*}}=t_{k} \cdot\left(M_{s s}-M_{0}\right) \cdot e^{-R_{1}^{*} \cdot t_{k}}
\end{aligned}
$$

where ${ }^{*}$ denotes pointwise complex conjugation. 



\section{Summary and Outlook}

\subsection{Summary}

The major goal of this thesis was the development of advanced single-shot $T_{1}$ mapping techniques offering experimental robustness, accuracy and precision, and high spatial resolution. Based on the inversion recovery Look-Locker sequence, this goal could be achieved by two complementary developments using either a modified real-time MRI technique with subsequent $T_{1}$ fitting or an advanced model-based reconstruction technique for direct $T_{1}$ mapping with even more flexible data acquisition schemes.

The first method relies on highly undersampled radial FLASH acquisitions and an image reconstruction involving two separate steps, i.e., nonlinear inverse estimation of optimal coil sensitivities from the steady-state signals using NLINV and subsequent image reconstruction using an iteratively regularized linear reconstruction method based on the $\mathrm{CG}$ algorithm. The proposed method offers substantial freedom of user-selective choices of spatial and temporal resolution. Moreover, it has been extended towards multi-slice $T_{1}$ mapping yielding simultaneous $T_{1}$ maps for up to 5 sections. Further, as $T_{1}$ values of the myocardium are of utmost clinical interest, a single-slice version of the above method was adapted for single-shot diastolic myocardial $T_{1}$ mapping where images from systolic phases are automatically deleted prior to pixel-wise fitting. In contrast to other existing methods such as MOLLI, the proposed method demonstrates better accuracy and higher spatial resolution within a much shorter acquisition time.

The second methodological contribution is the development of a model-based reconstruction technique which directly estimates parameter maps and coil sensitivities from the raw data. The algorithm can be considered as an extension of NLINV as the inversion-recovery signal model contains more parameters than required for parallel imaging (i.e., image content and coil sensitivities). With use of IRGNM for solving the nonlinear problem, the algorithm for the linearized subproblem was modified from CG to FISTA to incorporate a joint sparsity regularization for improving the precision of the parameter maps. Further, in order to deal with the high computational demand, a parallelized implementation of the above algorithms in C/CUDA has been accomplished. Numerical as well as experimental 
results indicate a significant noise suppression when replacing the Tikhonov regularization with the sparsity constraints in the model-based reconstruction. When compared to the image-space method based on real-time MRI, the sparsity regularized model-based reconstruction reveals a better balance between noise reduction and preservation of details.

In summary, both methods developed in this thesis allow for fast and accurate $T_{1}$ mapping using data from only a single inversion recovery experiment. Applications of the first method could successfully be extended from static to moving tissues, i.e., achieving single-shot myocardial $T_{1}$ mapping. Simulations as well as first results for an experimental phantom and human subjects (brain, abdomen) suggest superiority of the second method, i.e., a model-based reconstruction. However, further optimizations and more extensive validations are still needed.

\subsection{Future Work}

A major drawback of the model-based reconstruction is the increased computation time. Although the main optimization part has been implemented in C/CUDA, the communication between CPU and GPU still largely prolongs the computation. Further optimization of the parallelized implementation could yield an acceleration factor of at least 2-5. In addition, the possibility of algorithmic improvements should be further investigated, e.g., it is possible to build a preconditioner dynamically during IRGNM iterations to speed up the algorithm [124]. There are also several ways to avoid the expensive computation of Lipschitz constants used in FISTA [125].

Other physical measures could be the investigation of a more accurate modeling of the inversion-recovery process such as the incorporation of inversion efficiency and partial volume effects. So far, masking of systolic images in myocardial $T_{1}$ mapping relies on the signal from the finger pulse. A data-driven automatic sorting of systolic/diastolic data based on the information implicitly contained in the raw data may be developed to make this technique an even more robust clinical tool.

The application of the model-based reconstruction technique in single-shot myocardial mapping is another open question. The combination of a very short acquisition time, high accuracy and precision and the lack of postprocessing render this method an attractive alternative to existing methods [91]. Further, the algorithms developed for the sparsity regularization in the model-based reconstruction can also easily be adopted in other applications based on the IRGNM algorithm. Possible examples comprise diffusion imaging using NLINV or model-based reconstructions for parameters such as flow velocity or $T_{2}$ to improve the conditioning of corresponding inverse problems. 
Finally, after further optimizations as discussed above, it will be necessary to evaluate the methods based on image space (i.e., real-time MRI with pixelwise fitting) and data space (i.e., model-based reconstructions) more extensively in clinical settings such as $T_{1}$ mapping of abnormal tissues or myocardial $T_{1}$ mapping before and after contrast agent in order to appraise their values for diagnostic procedures. 



\section{Appendix}

\section{Formulation of IR Look-Locker Signal Model}

Suppose the longitudinal magnetization is $m_{i}$ before each $\alpha$ pulse. After the $\alpha$ pulse, it becomes $m_{i} \cos (\alpha)$, during the readout, the longitudinal magnetization will regrow according to $m(t)=1+[m(0)-1] \exp \left(-t / T_{1}\right)$. Therefore, the longitudinal magnetization before the next pulse is

$$
m_{i+1}=1+\left[m_{i} \cos (\alpha)-1\right] \exp \left(-\mathrm{TR} / T_{1}\right)
$$

When an inversion pulse is applied, i.e., $m(0)=-1$, by relating $m_{i}$ to $m_{0}$ with induction, equation (.1) has an explicit expression:

$$
m_{i}=m_{\infty}-\left(1+m_{\infty}\right)\left[\cos (\alpha) \exp \left(-\mathrm{TR} / T_{1}\right)\right]^{i}
$$

with

$$
m_{\infty}=\frac{1-\exp \left(-\mathrm{TR} / T_{1}\right)}{1-\cos (\alpha) \exp \left(-\mathrm{TR} / T_{1}\right)}
$$

and $i$ the index of the $i$ th $\alpha$ pulse. Because $t=i \mathrm{TR}$, by substituting $i=t / \mathrm{TR}$ and reformulation, equation $(.2)$ will become

$$
m(t)=m_{\infty}-\left(1+m_{\infty}\right) \exp \left(-t / T_{1}^{*}\right)
$$

with an effective relaxation time $T_{1}^{*}$ given by

$$
\frac{1}{T_{1}^{*}}=\frac{1}{T_{1}}-\frac{1}{\mathrm{TR}} \ln [\cos (\alpha)]
$$

and

$$
m_{\infty}=\frac{1-\exp \left(-\mathrm{TR} / T_{1}\right)}{1-\exp \left(-\mathrm{TR} / T_{1}^{*}\right)}
$$

Since normally $\mathrm{TR} \ll T_{1}^{*}, T_{1}, m_{\infty}$ can be approximated by

$$
m_{\infty} \approx \frac{T_{1}^{*}}{T_{1}}
$$


Therefore, the signal equation of the Look-Locker sequence can be described by

$$
M(t)=M_{s s}-\left(M_{0}+M_{s s}\right) \exp \left(-t / T_{1}^{*}\right)
$$

\section{Analytical Fourier Transform for Phantom Simulations}

Simulations of MRI samples have achieved by constructing phantoms from a composition of primitives, from which the continuous Fourier transform ( $(\overline{F T})$ is known analytically. The FT of a rect function is known to be a sinc function. Accordingly, the $k$-space of a two-dimensional rectangle can be calculated from:

$$
f_{\text {rect }}\left(k_{x}, k_{y}\right)=2 \pi \cdot a_{x} \cdot a_{y} \cdot \operatorname{sinc}\left(a_{x} \cdot k_{x}\right) \cdot \operatorname{sinc}\left(a_{y} \cdot k_{y}\right)
$$

where $a_{x}, a_{y}$ denote the size of the rectangle in $x$ - and $y$-direction. Similarly, the 2D FT of an ellipse can also be expressed analytically. This expression allows to simulate samples from numerical phantoms with elliptical primitives. The respective $k$-space samples can be calculated by:

$$
f_{\text {circ }}\left(k_{x}, k_{y}\right)=\frac{a \cdot J_{1}\left(b \cdot \sqrt{\left(a / b \cdot k_{x}\right)^{2}+k_{y}^{2}}\right)}{\sqrt{\left(a / b \cdot k_{x}\right)^{2}+k_{y}^{2}}}
$$

with $J_{1}(\cdot)$ the first-order Bessel function of the first kind and a, b are the axes of the ellipse [126]. Due to the linearity of the Fourier transformation, an analytical FT of any composition of rectangles and ellipses can be derived by superposition of these functions, where a displacement of a single element can be realized with a linear phase modulation. Therefore, arbitrary numerical phantoms can be constructed for the simulations [15]. 


\section{Bibliography}

[1] E. M. Haacke, R. W. Brown, M. R. Thompson, R. Venkatesan, et al., Magnetic resonance imaging: physical principles and sequence design, vol. 82. WileyLiss New York:, 1999.

[2] Z.-P. Liang and P. C. Lauterbur, Principles of magnetic resonance imaging: a signal processing perspective. The Institute of Electrical and Electronics Engineers Press, 2000.

[3] M. A. Bernstein, K. F. King, and X. J. Zhou, Handbook of MRI pulse sequences. Elsevier, 2004.

[4] I. I. Rabi, "Space quantization in a gyrating magnetic field," Physical Review, vol. 51, no. 8, p. 652, 1937.

[5] F. Bloch, "Nuclear induction," Physical Review, vol. 70, no. 7-8, p. 460, 1946.

[6] E. M. Purcell, H. Torrey, and R. V. Pound, "Resonance absorption by nuclear magnetic moments in a solid," Physical Review, vol. 69, no. 1-2, p. 37, 1946.

[7] P. C. Lauterbur, "Image formation by induced local interactions: examples employing nuclear magnetic resonance," Nature, vol. 242, pp. 190-191, 1973.

[8] C. P. Slichter, Principles of magnetic resonance, vol. 1. Springer Science \& Business Media, 2013.

[9] A. Haase, J. Frahm, D. Matthaei, W. Hanicke, and K.-D. Merboldt, "FLASH imaging. Rapid NMR imaging using low flip-angle pulses," Journal of Magnetic Resonance, vol. 67, no. 2, pp. 258-266, 1986.

[10] E. L. Hahn, "Spin echoes," Physical Review, vol. 80, no. 4, p. 580, 1950.

[11] M. Silver, R. Joseph, and D. Hoult, "Highly selective $\pi 2$ and $\pi$ pulse generation," Journal of Magnetic Resonance, vol. 59, no. 2, pp. 347-351, 1984. 
[12] C. Hardy, W. Edelstein, and D. Vatis, "Efficient adiabatic fast passage for NMR population inversion in the presence of radiofrequency field inhomogeneity and frequency offsets," Journal of Magnetic Resonance, vol. 66, no. 3, pp. 470-482, 1986.

[13] D. Rosenfeld and Y. Zur, "A new adiabatic inversion pulse," Magnetic Resonance in Medicine, vol. 36, no. 1, pp. 124-136, 1996.

[14] R. J. Ordidge, M. Wylezinska, J. W. Hugg, E. Butterworth, and F. Franconi, "Frequency offset corrected inversion (FOCI) pulses for use in localized spectroscopy," Magnetic Resonance in Medicine, vol. 36, no. 4, pp. 562-566, 1996.

[15] K. T. Block, Advanced methods for radial data sampling in magnetic resonance imaging. PhD thesis, 2008.

[16] K. T. Block and J. Frahm, "Spiral imaging: a critical appraisal," Journal of Magnetic Resonance Imaging, vol. 21, no. 6, pp. 657-668, 2005.

[17] E. J. Candès, J. Romberg, and T. Tao, "Robust uncertainty principles: Exact signal reconstruction from highly incomplete frequency information," Information Theory, IEEE Transactions on, vol. 52, no. 2, pp. 489-509, 2006.

[18] M. Lustig, D. Donoho, and J. M. Pauly, "Sparse MRI: The application of compressed sensing for rapid MR imaging," Magnetic Resonance in Medicine, vol. 58, no. 6, pp. 1182-1195, 2007.

[19] A. V. Oppenheim and R. W. Schafer, Discrete-time signal processing. Pearson Higher Education, 2010.

[20] C. E. Shannon, "Communication in the presence of noise," Proceedings of the IRE, vol. 37, no. 1, pp. 10-21, 1949.

[21] S. Zhang, K. T. Block, and J. Frahm, "Magnetic resonance imaging in real time: advances using radial FLASH," Journal of Magnetic Resonance Imaging, vol. 31, no. 1, pp. 101-109, 2010.

[22] F. Aurenhammer, "Voronoi diagrams - a survey of a fundamental geometric data structure," ACM Computing Surveys (CSUR), vol. 23, no. 3, pp. 345405, 1991.

[23] J. Nocedal and S. Wright, Numerical optimization. Springer Science \& Business Media, 2006. 
[24] P. B. Roemer, W. A. Edelstein, C. E. Hayes, S. P. Souza, and O. M. Mueller, "The NMR phased array," Magnetic Resonance in Medicine, vol. 16, no. 2, pp. 192-225, 1990.

[25] D. K. Sodickson and W. J. Manning, "Simultaneous acquisition of spatial harmonics (SMASH): fast imaging with radiofrequency coil arrays," Magnetic Resonance in Medicine, vol. 38, no. 4, pp. 591-603, 1997.

[26] K. P. Pruessmann, M. Weiger, M. B. Scheidegger, P. Boesiger, et al., "SENSE: sensitivity encoding for fast MRI," Magnetic Resonance in Medicine, vol. 42, no. 5, pp. 952-962, 1999.

[27] K. P. Pruessmann, M. Weiger, P. Börnert, and P. Boesiger, "Advances in sensitivity encoding with arbitrary k-space trajectories," Magnetic Resonance in Medicine, vol. 46, no. 4, pp. 638-651, 2001.

[28] P. M. Jakob, M. A. Grisowld, R. R. Edelman, and D. K. Sodickson, "AUTOSMASH: a self-calibrating technique for SMASH imaging," Magnetic Resonance Materials in Physics, Biology and Medicine, vol. 7, no. 1, pp. 42-54, 1998.

[29] M. A. Griswold, P. M. Jakob, R. M. Heidemann, M. Nittka, V. Jellus, J. Wang, B. Kiefer, and A. Haase, "Generalized autocalibrating partially parallel acquisitions (GRAPPA)," Magnetic Resonance in Medicine, vol. 47, no. 6, pp. 1202-1210, 2002.

[30] M. Lustig and J. M. Pauly, "SPIRiT: Iterative self-consistent parallel imaging reconstruction from arbitrary k-space," Magnetic Resonance in Medicine, vol. 64, no. 2, pp. 457-471, 2010.

[31] M. Uecker, P. Lai, M. J. Murphy, P. Virtue, M. Elad, J. M. Pauly, S. S. Vasanawala, and M. Lustig, "ESPIRiT-an eigenvalue approach to autocalibrating parallel MRI: where SENSE meets GRAPPA," Magnetic Resonance in Medicine, vol. 71, no. 3, pp. 990-1001, 2014.

[32] R. M. Heidemann, Ö. Özsarlak, P. M. Parizel, J. Michiels, B. Kiefer, V. Jellus, M. Müller, F. Breuer, M. Blaimer, M. A. Griswold, et al., "A brief review of parallel magnetic resonance imaging," European Radiology, vol. 13, no. 10, pp. 2323-2337, 2003.

[33] M. Blaimer, F. Breuer, M. Mueller, R. M. Heidemann, M. A. Griswold, and P. M. Jakob, "SMASH, SENSE, PILS, GRAPPA: how to choose the optimal method," Topics in Magnetic Resonance Imaging, vol. 15, no. 4, pp. 223-236, 2004. 
[34] M. Uecker, Nonlinear reconstruction methods for parallel magnetic resonance imaging. PhD thesis, Citeseer, 2009.

[35] K. P. Pruessmann, M. Weiger, M. B. Scheidegger, and P. Boesiger, "Coil sensitivity maps for sensitivity encoding and intensity correction," in Proceedings of the 6th Annual Meeting ISMRM, Sydney, Australia, p. 2087, 1998.

[36] K. T. Block, M. Uecker, and J. Frahm, "Undersampled radial MRI with multiple coils. iterative image reconstruction using a total variation constraint," Magnetic Resonance in Medicine, vol. 57, no. 6, pp. 1086-1098, 2007.

[37] D. Liang, B. Liu, J. Wang, and L. Ying, "Accelerating SENSE using compressed sensing," Magnetic Resonance in Medicine, vol. 62, no. 6, pp. 15741584, 2009.

[38] R. Otazo, D. Kim, L. Axel, and D. K. Sodickson, "Combination of compressed sensing and parallel imaging for highly accelerated first-pass cardiac perfusion MRI," Magnetic Resonance in Medicine, vol. 64, no. 3, pp. 767-776, 2010 .

[39] E. N. Yeh, C. A. McKenzie, M. A. Ohliger, and D. K. Sodickson, "Parallel magnetic resonance imaging with adaptive radius in k-space (PARS): Constrained image reconstruction using k-space locality in radiofrequency coil encoded data," Magnetic Resonance in Medicine, vol. 53, no. 6, pp. 13831392, 2005.

[40] D. O. Walsh, A. F. Gmitro, and M. W. Marcellin, "Adaptive reconstruction of phased array MR imagery," Magnetic Resonance in Medicine, vol. 43, no. 5, pp. 682-690, 2000.

[41] H. Gudbjartsson and S. Patz, "The Rician distribution of noisy MRI data," Magnetic Resonance in Medicine, vol. 34, no. 6, pp. 910-914, 1995.

[42] C. D. Constantinides, E. Atalar, and E. R. McVeigh, "Signal-to-noise measurements in magnitude images from NMR phased arrays," Magnetic Resonance in Medicine, vol. 38, no. 5, pp. 852-857, 1997.

[43] P. Mansfield, "Real-time echo-planar imaging by NMR," British Medical Bulletin, vol. 40, no. 2, pp. 187-190, 1984.

[44] K. S. Nayak, C. H. Cunningham, J. M. Santos, and J. M. Pauly, "Real-time cardiac MRI at 3 tesla," Magnetic Resonance in Medicine, vol. 51, no. 4, pp. 655-660, 2004. 
[45] J. Frahm, A. Haase, and D. Matthaei, "Rapid NMR imaging of dynamic processes using the FLASH technique," Magnetic Resonance in Medicine, vol. 3, no. 2, pp. 321-327, 1986.

[46] L. Ying and J. Sheng, "Joint image reconstruction and sensitivity estimation in SENSE (JSENSE)," Magnetic Resonance in Medicine, vol. 57, no. 6, pp. 1196-1202, 2007.

[47] M. Uecker, T. Hohage, K. T. Block, and J. Frahm, "Image reconstruction by regularized nonlinear inversion - joint estimation of coil sensitivities and image content," Magnetic Resonance in Medicine, vol. 60, no. 3, pp. 674682, 2008.

[48] H. W. Engl, M. Hanke, and A. Neubauer, Regularization of inverse problems, vol. 375. Springer Science \& Business Media, 1996.

[49] M. Uecker, S. Zhang, D. Voit, A. Karaus, K.-D. Merboldt, and J. Frahm, "Real-time MRI at a resolution of $20 \mathrm{ms,"} \mathrm{NMR} \mathrm{in} \mathrm{Biomedicine,} \mathrm{vol.} \mathrm{23,}$ no. 8, pp. 986-994, 2010.

[50] M. Uecker, S. Zhang, and J. Frahm, "Nonlinear inverse reconstruction for real-time MRI of the human heart using undersampled radial FLASH," Magnetic Resonance in Medicine, vol. 63, no. 6, pp. 1456-1462, 2010.

[51] S. Schaetz and M. Uecker, "A multi-GPU programming library for real-time applications," in International Conference on Algorithms and Architectures for Parallel Processing, pp. 114-128, Springer, 2012.

[52] K. Block and M. Uecker, "Simple method for adaptive gradient-delay compensation in radial MRI," in Proceedings of the 19th Annual Meeting ISMRM, Montreal, Quebec, Canada, p. 2816, 2011.

[53] F. Wajer and K. Pruessmann, "Major speedup of reconstruction for sensitivity encoding with arbitrary trajectories," in Proceedings of the 9th Annual Meeting ISMRM, Glasgow, Scotland, UK, p. 767, 2001.

[54] J. Klosowski and J. Frahm, "Image Denoising for Real-Time MRI," Magnetic Resonance in Medicine, 2016.

[55] H.-L. Margaret Cheng, N. Stikov, N. R. Ghugre, and G. A. Wright, "Practical medical applications of quantitative MR relaxometry," Journal of Magnetic Resonance Imaging, vol. 36, no. 4, pp. 805-824, 2012.

[56] P. Tofts, Quantitative MRI of the brain: measuring changes caused by disease. John Wiley \& Sons, 2005. 
[57] R. W. Brown, Y.-C. N. Cheng, E. M. Haacke, M. R. Thompson, and R. Venkatesan, Magnetic resonance imaging: physical principles and sequence design. John Wiley \& Sons, 2014.

[58] L. Drain, "A direct method of measuring nuclear spin-lattice relaxation times," Proceedings of the Physical Society. Section A, vol. 62, no. 5, p. 301, 1949.

[59] E. L. Hahn, "An accurate nuclear magnetic resonance method for measuring spin-lattice relaxation times," Physical Review, vol. 76, no. 1, p. 145, 1949.

[60] J. K. Barral, E. Gudmundson, N. Stikov, M. Etezadi-Amoli, P. Stoica, and D. G. Nishimura, "A robust methodology for in vivo T1 mapping," Magnetic Resonance in Medicine, vol. 64, no. 4, pp. 1057-1067, 2010.

[61] E. D. Becker, J. A. Ferretti, R. K. Gupta, and G. H. Weiss, "The choice of optimal parameters for measurement of spin-lattice relaxation times. ii. comparison of saturation recovery, inversion recovery, and fast inversion recovery experiments," Journal of Magnetic Resonance, vol. 37, no. 3, pp. 381394, 1980.

[62] D. C. Look and D. R. Locker, "Time saving in measurement of NMR and EPR relaxation times," Review of Scientific Instruments, vol. 41, no. 2, pp. 250$251,1970$.

[63] A. P. Crawley and R. M. Henkelman, "A comparison of one-shot and recovery methods in T1 imaging," Magnetic Resonance in Medicine, vol. 7, no. 1, pp. 23-34, 1988.

[64] R. Deichmann and A. Haase, "Quantification of T1 values by SNAPSHOTFLASH NMR imaging," Journal of Magnetic Resonance, vol. 96, no. 3, pp. 608-612, 1992.

[65] P. Gowland and P. Mansfield, "Accurate measurement of T1 in vivo in less than 3 seconds using echo-planar imaging," Magnetic Resonance in Medicine, vol. 30, no. 3, pp. 351-354, 1993.

[66] K. Scheffler and J. Hennig, "T1 quantification with inversion recovery TrueFISP," Magnetic Resonance in Medicine, vol. 45, no. 4, pp. 720-723, 2001.

[67] E. K. Fram, R. J. Herfkens, G. A. Johnson, G. H. Glover, J. P. Karis, A. Shimakawa, T. G. Perkins, and N. J. Pelc, "Rapid calculation of T1 using variable flip angle gradient refocused imaging," Magnetic Resonance Imaging, vol. 5, no. 3, pp. 201-208, 1987. 
[68] S. C. Deoni, T. M. Peters, and B. K. Rutt, "High-resolution T1 and T2 mapping of the brain in a clinically acceptable time with DESPOT1 and DESPOT2," Magnetic Resonance in Medicine, vol. 53, no. 1, pp. 237-241, 2005.

[69] S. C. Deoni, T. M. Peters, and B. K. Rutt, "Determination of optimal angles for variable nutation proton magnetic spin-lattice, T1, and spin-spin, T2, relaxation times measurement," Magnetic Resonance in Medicine, vol. 51, no. 1, pp. 194-199, 2004.

[70] C. H. Cunningham, J. M. Pauly, and K. S. Nayak, "Saturated double-angle method for rapid B1 + mapping," Magnetic Resonance in Medicine, vol. 55, no. 6, pp. 1326-1333, 2006.

[71] V. L. Yarnykh, "Actual flip-angle imaging in the pulsed steady state: a method for rapid three-dimensional mapping of the transmitted radiofrequency field," Magnetic Resonance in Medicine, vol. 57, no. 1, pp. 192-200, 2007.

[72] L. I. Sacolick, F. Wiesinger, I. Hancu, and M. W. Vogel, "B1 mapping by Bloch-Siegert shift," Magnetic Resonance in Medicine, vol. 63, no. 5, pp. 1315-1322, 2010.

[73] S. Winkelmann, T. Schaeffter, T. Koehler, H. Eggers, and O. Doessel, "An optimal radial profile order based on the Golden Ratio for time-resolved MRI," Medical Imaging, IEEE Transactions on, vol. 26, no. 1, pp. 68-76, 2007.

[74] P. Ehses, N. Seiberlich, D. Ma, F. A. Breuer, P. M. Jakob, M. A. Griswold, and V. Gulani, "IR TrueFISP with a golden-ratio-based radial readout: Fast quantification of T1, T2, and proton density," Magnetic Resonance in Medicine, vol. 69, no. 1, pp. 71-81, 2013.

[75] H. K. Song and L. Dougherty, "k-space weighted image contrast (KWIC) for contrast manipulation in projection reconstruction MRI," Magnetic Resonance in Medicine, vol. 44, no. 6, pp. 825-832, 2000.

[76] J. Tran-Gia, D. Stäb, T. Wech, D. Hahn, and H. Köstler, "Model-based Acceleration of Parameter mapping (MAP) for saturation prepared radially acquired data," Magnetic Resonance in Medicine, vol. 70, no. 6, pp. 15241534, 2013. 
[77] S. Zhang, M. Uecker, D. Voit, K.-D. Merboldt, and J. Frahm, "Real-time cardiovascular magnetic resonance at high temporal resolution: radial FLASH with nonlinear inverse reconstruction," Journal of Cardiovascular Magnetic Resonance, vol. 12, no. 1, p. 39, 2010.

[78] V. Roeloffs, D. Voit, and J. Frahm, "Spoiling without additional gradients: Radial FLASH MRI with randomized radiofrequency phases," Magnetic Resonance in Medicine, vol. 75, no. 5, pp. 2094-2099, 2015.

[79] T. Sumpf and M. Unterberger, "arrayshow: a guide to an open source Matlab tool for complex MRI data analysis," in Proceedings of the 21st Annual Meeting ISMRM, Salt Lake City, Utah, USA, p. 2719, 2013.

[80] M. Guerquin-Kern, L. Lejeune, K. P. Pruessmann, and M. Unser, "Realistic analytical phantoms for parallel magnetic resonance imaging," Medical Imaging, IEEE Transactions on, vol. 31, no. 3, pp. 626-636, 2012.

[81] X. Wang, V. B. Roeloffs, K. D. Merboldt, D. Voit, S. Schätz, and J. Frahm, "Single-shot multi-slice T1 mapping at high spatial resolution - inversionrecovery FLASH with radial undersampling and iterative reconstruction.," The Open Medical Imaging Journal, vol. 9, pp. 1-8, 2015.

[82] G. E. Santyr, E. J. Fairbanks, F. Kelcz, and J. A. Sorenson, "Off-resonance spin locking for MR imaging," Magnetic Resonance in Medicine, vol. 32, no. 1, pp. 43-51, 1994.

[83] G. Liberman, Y. Louzoun, and D. Ben Bashat, "T1 mapping using variable flip angle SPGR data with flip angle correction," Journal of Magnetic Resonance Imaging, vol. 40, no. 1, pp. 171-180, 2014.

[84] M. Haimerl, N. Verloh, F. Zeman, C. Fellner, R. Müller-Wille, A. G. Schreyer, C. Stroszczynski, and P. Wiggermann, "Assessment of clinical signs of liver cirrhosis using T1 mapping on Gd-EOB-DTPA-enhanced 3T MRI," PloS one, vol. 8, no. 12, p. e85658, 2013.

[85] K. A. Gillis, C. McComb, J. E. Foster, A. H. Taylor, R. K. Patel, S. T. Morris, A. G. Jardine, M. P. Schneider, G. H. Roditi, C. Delles, et al., "Interstudy reproducibility of arterial spin labelling magnetic resonance imaging for measurement of renal perfusion in healthy volunteers at 3 tesla," BMC Nephrology, vol. 15, no. 1, p. 1, 2014.

[86] M. Kunth, N. Seiberlich, P. Ehses, V. Gulani, and M. Griswold, "Improvement of quantitative MRI using radial GRAPPA in conjunction with IR- 
TrueFISP," in Proceedings of the 18th Annual Meeting ISMRM, Stockholm, Sweden, p. 2895, 2010.

[87] J. Tran-Gia, T. Wech, T. Bley, and H. Köstler, "Model-based acceleration of Look-Locker T1 mapping," PloS one, vol. 10, no. 4, p. e0122611, 2015.

[88] A. Haase, "Snapshot FLASH MRI. Applications to T1, T2, and chemical-shift imaging," Magnetic Resonance in Medicine, vol. 13, no. 1, pp. 77-89, 1990.

[89] R. Ordidge, P. Gibbs, B. Chapman, M. Stehling, and P. Mansfield, "Highspeed multislice T1 mapping using inversion-recovery echo-planar imaging," Magnetic Resonance in Medicine, vol. 16, no. 2, pp. 238-245, 1990.

[90] D. R. Messroghli, A. Radjenovic, S. Kozerke, D. M. Higgins, M. U. Sivananthan, and J. P. Ridgway, "Modified Look-Locker inversion recovery (MOLLI) for high-resolution T1 mapping of the heart," Magnetic Resonance in Medicine, vol. 52, no. 1, pp. 141-146, 2004.

[91] P. Kellman and M. S. Hansen, "T1-mapping in the heart: accuracy and precision," Journal of Cardiovascular Magnetic Resonance, vol. 16, no. 2, 2014.

[92] J. C. Moon, D. R. Messroghli, P. Kellman, S. K. Piechnik, M. D. Robson, M. Ugander, P. D. Gatehouse, A. E. Arai, M. G. Friedrich, S. Neubauer, et al., "Myocardial T1 mapping and extracellular volume quantification: a society for cardiovascular magnetic resonance (SCMR) and CMR working group of the european society of cardiology consensus statement," Journal of Cardiovascular Magnetic Resonance, vol. 15, no. 1, p. 1, 2013.

[93] S. Hofer, X. Wang, V. Roeloffs, and J. Frahm, "Single-shot T1 mapping of the corpus callosum: a rapid characterization of fiber bundle anatomy," Frontiers in Neuroanatomy, vol. 9, pp. 1-6, 2015.

[94] D. Voit, S. Zhang, C. Unterberg-Buchwald, J. M. Sohns, J. Lotz, and J. Frahm, "Real-time cardiovascular magnetic resonance at $1.5 \mathrm{~T}$ using balanced SSFP and 40 ms resolution," Journal of Cardiovascular Magnetic Resonance, vol. 15, p. 79, 2013.

[95] M. Untenberger, Z. Tan, D. Voit, A. Joseph, V. Roeloffs, K. Merboldt, S. Schätz, and J. Frahm, "Advances in real-time phase-contrast flow MRI using asymmetric radial gradient echoes.," Magnetic Resonance in Medicine, vol. 75, no. 5, pp. 1901-8, 2016. 
[96] S. Zhang, A. Joseph, D. Voit, S. Schätz, K. Merboldt, C. UnterbergBuchwald, A. Hennemuth, J. Lotz, and J. Frahm, "Real-time MRI of cardiac function and flow - Recent progress.," Quantitative Imaging in Medicine and Surgery, vol. 4, pp. 313-329, 2014.

[97] J. Frahm, S. Schätz, M. Untenberger, S. Zhang, D. Voit, K. D. Merboldt, J. M. Sohns, J. Lotz, and M. Uecker, "On the temporal fidelity of nonlinear inverse reconstructions for real-time MRI - The motion challenge.," The Open Medical Imaging Journal, vol. 8, pp. 1-7, 2014.

[98] D. E. King, "Dlib-ml: A Machine Learning Toolkit," Journal of Machine Learning Research, vol. 10, pp. 1755-1758, 2009.

[99] R. Deichmann, "Fast high-resolution T1 mapping of the human brain," Magnetic Resonance in Medicine, vol. 54, no. 1, pp. 20-27, 2005.

[100] F. von Knobelsdorff-Brenkenhoff, M. Prothmann, M. A. Dieringer, R. Wassmuth, A. Greiser, C. Schwenke, T. Niendorf, and J. Schulz-Menger, "Myocardial $\mathrm{T} 1$ and $\mathrm{T} 2$ mapping at $3 \mathrm{~T}$ : reference values, influencing factors and implications," Journal of Cardiovascular Magnetic Resonance, vol. 15, no. 1, p. 53, 2013.

[101] J. J. Lee, S. Liu, M. S. Nacif, M. Ugander, J. Han, N. Kawel, C. T. Sibley, P. Kellman, A. E. Arai, and D. A. Bluemke, "Myocardial T1 and extracellular volume fraction mapping at 3 tesla," Journal of Cardiovascular Magnetic Resonance, vol. 13, no. 1, p. 75, 2011.

[102] N. Kawel, M. Nacif, A. Zavodni, J. Jones, S. Liu, C. T. Sibley, and D. A. Bluemke, "T1 mapping of the myocardium: intra-individual assessment of the effect of field strength, cardiac cycle and variation by myocardial region," Journal of Cardiovascular Magnetic Resonance, vol. 14, no. 1, p. 27, 2012.

[103] J. Shao, S. Rapacchi, K.-L. Nguyen, and P. Hu, "Myocardial T1 mapping at 3.0 tesla using an inversion recovery spoiled gradient echo readout and bloch equation simulation with slice profile correction (BLESSPC) T1 estimation algorithm," Journal of Magnetic Resonance Imaging, vol. 43, no. 2, pp. 414-425, 2016.

[104] D. Gensler, P. Mörchel, F. Fidler, O. Ritter, H. H. Quick, M. E. Ladd, W. R. Bauer, G. Ertl, P. M. Jakob, and P. Nordbeck, "Myocardial T1: Quantification by using an ECG-triggered radial single-shot inversion-recovery MR imaging sequence," Radiology, vol. 274, no. 3, pp. 879-887, 2015. 
[105] B. B. Mehta, X. Chen, K. C. Bilchick, M. Salerno, and F. H. Epstein, "Accelerated and navigator-gated Look-Locker imaging for cardiac T1 estimation (ANGIE): Development and application to T1 mapping of the right ventricle," Magnetic Resonance in Medicine, vol. 73, no. 1, pp. 150-160, 2015.

[106] M. Doneva, P. Börnert, H. Eggers, C. Stehning, J. Sénégas, and A. Mertins, "Compressed sensing reconstruction for magnetic resonance parameter mapping," Magnetic Resonance in Medicine, vol. 64, no. 4, pp. 1114-1120, 2010.

[107] F. H. Petzschner, I. P. Ponce, M. Blaimer, P. M. Jakob, and F. A. Breuer, "Fast MR parameter mapping using k-t principal component analysis," Magnetic Resonance in Medicine, vol. 66, no. 3, pp. 706-716, 2011.

[108] C. Huang, C. G. Graff, E. W. Clarkson, A. Bilgin, and M. I. Altbach, "T2 mapping from highly undersampled data by reconstruction of principal component coefficient maps using compressed sensing," Magnetic Resonance in Medicine, vol. 67, no. 5, pp. 1355-1366, 2012.

[109] J. V. Velikina, A. L. Alexander, and A. Samsonov, "Accelerating mr parameter mapping using sparsity-promoting regularization in parametric dimension," Magnetic resonance in medicine, vol. 70, no. 5, pp. 1263-1273, 2013.

[110] T. Zhang, J. M. Pauly, and I. R. Levesque, "Accelerating parameter mapping with a locally low rank constraint," Magnetic Resonance in Medicine, vol. 73, no. 2, pp. 655-661, 2015.

[111] B. Zhao, W. Lu, T. K. Hitchens, F. Lam, C. Ho, and Z.-P. Liang, "Accelerated MR parameter mapping with low-rank and sparsity constraints," Magnetic Resonance in Medicine, vol. 74, no. 2, pp. 489-498, 2015.

[112] V. T. Olafsson, D. C. Noll, and J. A. Fessler, "Fast joint reconstruction of dynamic and field maps in functional MRI," Medical Imaging, IEEE Transactions on, vol. 27, no. 9, pp. 1177-1188, 2008.

[113] K. T. Block, M. Uecker, and J. Frahm, "Model-based iterative reconstruction for radial fast spin-echo MRI," Medical Imaging, IEEE Transactions on, vol. 28, no. 11, pp. 1759-1769, 2009.

[114] T. J. Sumpf, M. Uecker, S. Boretius, and J. Frahm, "Model-based nonlinear inverse reconstruction for T2 mapping using highly undersampled spinecho MRI," Journal of Magnetic Resonance Imaging, vol. 34, no. 2, pp. 420428, 2011. 
[115] B. Zhao, F. Lam, and Z.-P. Liang, "Model-based MR parameter mapping with sparsity constraints: parameter estimation and performance bounds," Medical Imaging, IEEE Transactions on, vol. 33, no. 9, pp. 1832-1844, 2014.

[116] F. Knoll, J. G. Raya, R. O. Halloran, S. Baete, E. Sigmund, R. Bammer, T. Block, R. Otazo, and D. K. Sodickson, "A model-based reconstruction for undersampled radial spin-echo DTI with variational penalties on the diffusion tensor," NMR in Biomedicine, vol. 28, no. 3, pp. 353-366, 2015.

[117] Z. Tan, V. Roeloffs, D. Voit, A. A. Joseph, M. Untenberger, K. D. Merboldt, and J. Frahm, "Model-based reconstruction for real-time phasecontrast flow MRI: Improved spatiotemporal accuracy," Magnetic Resonance in Medicine, 2016.

[118] A. B. Bakushinsky and M. Y. Kokurin, Iterative methods for approximate solution of inverse problems, vol. 577. Springer Science \& Business Media, 2005.

[119] F. Knoll, C. Clason, K. Bredies, M. Uecker, and R. Stollberger, "Parallel imaging with nonlinear reconstruction using variational penalties," Magnetic Resonance in Medicine, vol. 67, no. 1, pp. 34-41, 2012.

[120] S. Vasanawala, M. Murphy, M. T. Alley, P. Lai, K. Keutzer, J. M. Pauly, and M. Lustig, "Practical parallel imaging compressed sensing MRI: Summary of two years of experience in accelerating body MRI of pediatric patients," in Biomedical Imaging: From Nano to Macro, 2011 IEEE International Symposium on, pp. 1039-1043, IEEE, 2011.

[121] A. Beck and M. Teboulle, "A fast iterative shrinkage-thresholding algorithm for linear inverse problems," SIAM Journal on Imaging Sciences, vol. 2, no. 1, pp. 183-202, 2009.

[122] M. Uecker, F. Ong, J. I. Tamir, D. Bahri, P. Virtue, J. Y. Cheng, T. Zhang, and M. Lustig, "Berkeley advanced reconstruction toolbox," in Proceedings of the 23rd Annual Meeting ISMRM, Toronto, Ontario, Canada, p. 2486, 2015.

[123] M. A. Figueiredo and R. D. Nowak, "An EM algorithm for wavelet-based image restoration," Image Processing, IEEE Transactions on, vol. 12, no. 8, pp. 906-916, 2003.

[124] T. Hohage, "On the numerical solution of a three-dimensional inverse medium scattering problem," Inverse Problems, vol. 17, no. 6, p. 1743, 2001. 
[125] F. Bach, R. Jenatton, J. Mairal, and G. Obozinski, "Optimization with sparsity-inducing penalties," Foundations and Trends $\cap$ in Machine Learning, vol. 4, no. 1, pp. 1-106, 2012.

[126] A. C. Kak and M. Slaney, Principles of computerized tomographic imaging. IEEE press, 1988. 



\section{Acronyms}

$T_{1}$ spin-lattice relaxation time.

$T_{2}$ spin-spin relaxation time.

$T_{2}^{*}$ effective spin-spin relaxation time.

ACS AutoCalibration Signal.

bSSFP balanced Steady State Free Precession.

CG Conjugate Gradient.

CG-SENSE Conjugate Gradient SENSE.

CMR Cardiovascular Magnetic Resonance.

CS Compressed Sensing.

CT Computed Tomography.

CUDA Compute Unified Device Architecture.

DCF Density Compensation Filter.

DFT Discrete Fourier Transformation.

EPI Echo Planner Imaging.

FFT Fast Fourier Transformation.

FID Free Induction Decay.

FISTA Fast Iterative Shrinkage-Thresholding Algorithm.

FLASH Fast Low Angle SHot.

FOV Field Of View.

FSE Fast Spin Echo. 
FT Fourier Transform.

GM Gray Matter.

GPU Graphic Processing Unit.

GRAPPA GeneRalized Autocalibrating Partially Parallel Acquisitions.

GRE Gradient Echo.

IR Inversion Recovery.

IRGNM Iteratively Regularized Gauss-Newton Method.

JSENSE Joint SENSE,

LL Look-Locker.

MOLLI Modified Look-Locker Inversion.

MRI Magnetic Resonance Imaging.

NLINV NonLinear INVersion.

NMR Nuclear Magnetic Resonance.

NUFFT Non-Uniform FFT,

PCA Principle Component Analysis.

PET Positron Emission Tomography.

RF Radio Frequency.

ROI Region Of Interest.

RSS Root of Sum of Squares.

SE Spin Echo.

SENSE SENSitivity Encoding.

SMASH SiMultaneous Acquisition of Spatial Harmonics.

SNR Signal-to-Noise Ratio.

SSFP Steady-State Free Precession. 
TE Echo Time.

TGV Total Generalized Variation.

TI Inversion Time.

TR Repetition Time.

TV Total Variation.

VFA Variable Flip Angle.

WM White Matter. 



\section{List of Publications}

\section{Journal Publications}

- Xiaoqing Wang, Volkert Roeloffs, Jacob Klosowski, Zhengguo Tan, Dirk Voit, Martin Uecker and Jens Frahm, Model-based $T_{1}$ Mapping with Sparsity Constraints Using Single-Shot Inversion-Recovery Radial FLASH, Magnetic Resonance in Medicine, doi: 10.1002/mrm.26726 (2017).

- Volkert Roeloffs, Xiaoqing Wang, Tilmann J Sumpf, Markus Untenberger, Dirk Voit and Jens Frahm, Model-based Reconstruction for $T_{1}$ Mapping using Single-shot Inversion-recovery Radial FLASH, International Journal of Imaging Systems and Technology, 26: 254-263 (2016).

- Xiaoqing Wang, Arun A Joseph, Oleksandr Kalentev, K Dietmar Merboldt, Dirk Voit, Volkert Roeloffs, Maaike van Zalk and Jens Frahm, High-resolution Myocardial $T_{1}$ Mapping using Single-shot Inversion-recovery Fast Low-Angle Shot MRI with Radial Undersampling and Iterative Reconstruction, The British Journal of Radiology, doi: 10.1259/bjr.20160255 (2016).

- Sabine Hofer, Xiaoqing Wang, Volkert Roeloffs and Jens Frahm, Single-shot $T_{1}$ Mapping of the Corpus Callosum: A Rapid Characterization of Fiber Bundle Anatomy, Frontiers in Neuroanatomy, 9: 1-6 (2015).

- Xiaoqing Wang, Volkert Roeloffs, K Dietmar Merboldt, Dirk Voit, Sebastian Schätz and Jens Frahm, Single-shot Multi-slice $T_{1}$ Mapping at High Spatial Resolution - Inversion Recovery FLASH with Radial Undersampling and Iterative Reconstruction, The Open Medical Imaging Journal, 9: 1-8 (2015).

\section{Conference Contributions}

- Volkert Roeloffs, Xiaoqing Wang and Jens Frahm, Advances in Model-Based Reconstruction of High Resolution $T_{1}$ maps, in Proceedings of the 24th Annual Meeting ISMRM, Singapore, 2016. 
- Xiaoqing Wang, Volkert Roeloffs, K Dietmar Merboldt, Dirk Voit, Sebastian Schätz and Jens Frahm, Single-shot Multi-slice $T_{1}$ Mapping at High Spatial Resolution - Inversion Recovery FLASH with Radial Undersampling and Iterative Reconstruction, in Proceedings of the 23th Annual Meeting ISMRM, Toronto, 2015.

- Volkert Roeloffs, Xiaoqing Wang and Jens Frahm, High Resolution $T_{1}$ mapping within Seconds: Model-based Reconstruction without Regularization, in Proceedings of the 23th Annual Meeting ISMRM, Toronto, 2015.

\section{Other Publications}

- Jiasong Wu, Xu Zhang, Xiaoqing Wang, Lotfi Senhadji, Huazhong Shu, $\ell_{1}$ norm Minimization for Quaternion Signals, Journal of Southeast University (English Version), 1: 33-37, 2013.

- Xiaoqing Wang, Jiasong Wu, Huazhong Shu, Two Dimensional Linear Convolution in Discrete Trigonometric Transform Domain, Journal of Southeast University (Chinese Version), 42 (3): 424-427, 2012. 


\section{Acknowledgements}

First and foremost I would like to express my sincere gratitude to Prof. Dr. Jens Frahm, head of the Biomedizinische NMR Forschungs GmbH am Max-PlanckInstitut für biophysikalische Chemie, for offering me this invaluable opportunity to pursue my $\mathrm{PhD}$ degree under his supervision, for providing me his continuous support, inspiring advices for the development of my research projects and also for his extensive assistance in the preparation of manuscripts during the work of this thesis. It has been a great honor and pleasure to be his student. His great vision, passion and enthusiasm about research have been highly inspirational and stimulating for me to pursue research as a $\mathrm{PhD}$ student. Besides the scientific part, I also learned leadership and management skills from him.

I am very grateful to Prof. Dr. Hans Hofsäs from the II. Institute of Physics at Georg-August-Universität Göttingen for being the examiner of my thesis and kind support provided during the work of this thesis.

I am most thankful to Drs. Klaus-Dietmar Merboldt, Dirk Voit, Shuo Zhang and Arun A. Joseph for the supervision of my PhD projects. They were always helpful and approachable for innumerable discussions, critical thinking and exploring new ideas. Specifically, many thanks to Dr. Voit for fruitful discussions about the sequence and MRI physics, to Dr. Merboldt and Dr. Zhang for guiding me into the field of real-time MRI as well as $T_{1}$ mapping, to Dr. Joseph for close collaborations in the project of myocardial $T_{1}$ mapping and numerous measurement assistance.

I also would like to express my appreciation to Drs. Oleksandr Kalentev, Dirk Voit and Maaike van Zalk for their efforts in integrating the $T_{1}$ mapping techniques presented in chapter 5 and chapter 6 into the pipeline of a commercial MRI system, which is really cool.

I want to thank my office-mate Dr. Volkert Roeloffs for close collaborations on the project of multi-slice $T_{1}$ mapping, for his critical suggestions on the development of new methods and for the knowledge exchange about eastern and western culture. I'm also thankful to the other former and present office-mates, Dr. Markus Untenberger, Dr. Zhengguo Tan, Jacob Klosowski and Jost Kollmeier. They are always patient in listening to my ideas and providing me insightful feedbacks. A special thank goes to Jost for teaching me "special" German.

Next, my sincere thank goes to Dr. Sabine Hofer for her assistance in improving 
the quality of this thesis as well as the close collaboration in the project of $T_{1}$ mapping of corpus callosum.

Further, I would like to thank all the other present and former staff members of the Biomedizinische NMR Forschungs GmbH (Arijit Hazra, Christian Holme, Andreas Merrem, Zhongshuai Zhang, to name a few) for a very pleasant working atmosphere and the enjoyable table-soccer sessions.

Last but not least, I am deeply indebted to my grandmother, my parents and my brother for the love, care and support through the journey of this thesis. Plus, I want to thank my girlfriend Zi Ying for her company, love and being always supportive during the hard days of my PhD. I also want to thank Zi's parents for their continuous encouragement. This thesis would not be possible without all of your support and understanding. 